\title{
Microhyperbolic Operators in Gevrey Classes
}

\author{
By \\ Kunihiko KaJITAni* and Seiichiro WaKabaYASHI*
}

\section{\$1. Introduction}

Kashiwara and Kawai [16] defined microhyperbolicity and proved that the microlocal Cauchy problem for microhyperbolic pseudodifferential operators is well-posed in the framework of microfunctions, which is a microlocalization of the results obtained by Bony and Schapira [3]. In the microlocal studies of pseudo-differential operators, the concept of microhyperbolicity is very useful. From their results one can obtain results on propagation of analytic singularities (propagation of micro-analyticities) of solutions for microhyperbolic operators (see [28]). On the other hand, Bronshtein [5] proved that the hyperbolic Cauchy problem is well-posed in some Gevrey classes which are intermediate spaces between the space of real analytic functions and $C^{\infty}$ (see, also, [14], [15]). So we can generalize the definition of microhyperbolicity in the framework of some Gevrey classes, to say the least of it. In doing so, we expect to get a clue to a generalization of microhyperbolicity and microlocal studies of microhyperbolic operators in the framework of $C^{\infty}$.

In this paper we shall consider microhyperbolic operators in Gevrey classes and prove microlocal well-posedness of the microlocal Cauchy problem and theorems on propagation of singularities for microhyperbolic operators. Our aims are to show how one can obtain microlocal results (microlocal well-posedness and, therefore, a microlocal version of Holmgren's uniqueness theorem) from methods to prove well-posedness of the Cauchy problem and to show that theorems on propagation of singularities are immediate consequences of a microlocal version of Holmgren's uniqueness theorem, using

Communicated by S. Matsuura, November 17, 1986.

* Institute of Mathematics, University of Tsukuba, Ibaraki 305, Japan. 
generalized Hamilton flows. We shall prove microlocal well-posedness, reducing the problems to those in $L^{2}$. From this point of view one may assert that consideration in $L^{2}$ (or $C^{\infty}$ ) are much more important than in Gevrey classes. However, in the framework of Gevrey classes one can easily solve some problems, which seem difficult to be solved in the framework of $C^{\infty}$, and obtain some conjectures on the problems in the framework of $C^{\infty}$. We should note that Uchikoshi [27] investigated a related problem.

Let $K$ be a regular compact set in $\boldsymbol{R}^{n}$, and let $\kappa>1$ and $h>0$. We denote by $\mathscr{E}^{[x], h}(K)$ the space of all $f \in C^{\infty}(K)$ which satisfies, with some constant $C \geq 0$,

$$
\left|D^{\alpha} f(x)\right| \leq C h^{|\alpha|}|\alpha| !^{\kappa} \text { for } x \in K \text { and }|\alpha|=0,1,2, \ldots,
$$

where $x=\left(x_{1}, \ldots, x_{n}\right) \in \boldsymbol{R}^{n}, D=i^{-1}\left(\partial / \partial x_{1}, \ldots, \partial / \partial x_{n}\right), \alpha=\left(\alpha_{1}, \ldots, \alpha_{n}\right)$ is a multi-index and $|\alpha|=\sum_{j=1}^{n} \alpha_{j}$. We also denote by $\mathscr{D}_{K}^{(\kappa), h}$ the space of all $f \in C^{\infty}\left(\boldsymbol{R}^{n}\right)$ with support in $K$ satisfying $(1.1) . \mathscr{E}^{(\kappa), h}(K)$ and $\mathscr{D}_{K}^{[\kappa], h}$ are Banach spaces under the norm defined by

$$
\|f\|_{\mathscr{E}^{(\kappa), h}(K)}=\sup _{x \in K, \alpha}\left|D^{\alpha} f(x)\right| /\left(h^{|\alpha|}|\alpha| !^{\kappa}\right) .
$$

Let $\Omega$ be an open set in $\boldsymbol{R}^{n}$. We introduce the following locally convex spaces (Gevrey classes):

$$
\begin{aligned}
& \mathscr{E}^{(k)}(\Omega)=\overleftarrow{K}_{K \subset \Omega}^{\lim } \mathscr{E}^{(k)}(K), \mathscr{E}^{(k)}(K)=\overleftarrow{\lim }_{h \rightarrow 0} \mathscr{E}^{(\kappa), h}(K), \\
& \mathscr{E}^{[\kappa]}(\Omega)={\underset{K}{K \subset \Omega}}_{\lim } \mathscr{E}^{(\kappa)}(K), \mathscr{E}^{(\kappa)}(K)={\underset{h \rightarrow \infty}{\lim }} \mathscr{E}^{(\kappa), h}(K), \\
& \mathscr{D}^{(\kappa)}(\Omega)=\underset{K \subset \Omega}{=\lim _{K}} \mathscr{D}_{K}^{(\kappa)}, \mathscr{D}_{K}^{(\kappa)}={\underset{\varliminf}{h \rightarrow 0}}_{K} \mathscr{D}_{K}^{(\kappa), h}, \\
& \mathscr{D}^{(\kappa)}(\Omega)=\underset{K \in \Omega}{=} \mathscr{\operatorname { l i m }}_{K}^{(\kappa)}, \mathscr{D}_{K}^{(\kappa)}=\underset{h \rightarrow \infty}{\lim } \mathscr{D}_{K}^{(\kappa), h},
\end{aligned}
$$

where $A \Subset B$ means that the closure $\bar{A}$ of $A$ is compact and included in the interior $\stackrel{\circ}{B}$ of $B$. We denote by $\mathscr{D}^{* \prime}(\Omega)$ and $\mathscr{E}^{*^{\prime}}(\Omega)$ the strong dual spaces of $\mathscr{D}^{*}(\Omega)$ and $\mathscr{E} *(\Omega)$, respectively, where $*$ denotes $(\kappa)$ or $\{\kappa\}$. We also write $\mathscr{E}^{*}, \ldots$, instead of $\mathscr{E}^{*}\left(\boldsymbol{R}^{n}\right), \ldots$ (see, e. g., [18]). Let us define symbol classes $S_{*}^{m}$, where $m \in \boldsymbol{R}$. We say that a symbol $p(x, \xi)$ belongs to $S_{(\kappa)}^{m}\left(\operatorname{resp} . S_{(\kappa)}^{m}\right)$ if $p(x, \xi) \in C^{\infty}\left(T^{*} \mathbb{R}^{n}\right)$ and for any compact subset $K$ of $\boldsymbol{R}^{n}$ and any $A>0$ there is $C \equiv C_{K, A}>0$ (resp. for any compact subset $K$ of $\boldsymbol{R}^{n}$ there are $A \equiv A_{K}>0$ and $\left.C \equiv C_{K}>0\right)$ such that 


$$
\left|p_{(\beta)}^{(\alpha)}(x, \xi)\right| \leq C A^{|\alpha|+|\beta|}(|\alpha|+|\beta|) !^{\kappa}\langle\xi\rangle^{m-|\alpha|}
$$

for $x \in K, \xi=\left(\xi_{1}, \ldots, \xi_{n}\right) \in \boldsymbol{R}^{n}$ and any multi-indeces $\alpha$ and $\beta$, where $T^{*} \boldsymbol{R}^{n}$ is identified with $\boldsymbol{R}^{n} \times \boldsymbol{R}^{n},\langle\xi\rangle=\left(1+|\xi|^{2}\right)^{1 / 2}$ and $p_{(\beta)}^{(\alpha)}(x, \xi)=$ $\partial_{\xi}^{\alpha} D_{x}^{\beta} p(x, \xi)$. We impose the following conditions:

(A-1) $\quad p(x, \xi) \in S_{* 1}^{m}$, where $* 1$ denotes $\left(\kappa_{1}\right)$ or $\left\{\kappa_{1}\right\}$, and $\kappa_{1}>1$ and $m \in \boldsymbol{R}$. And $p(x, D)$ is properly supported.

(A-2) There is a symbol $p_{m}(x, \xi)$, which is positively homogeneous of degree $m$ in $\xi$, such that $p(x, \xi)-\sigma(\xi) p_{m}(x, \xi) \in S_{* 1}^{m-1}$, $\sigma(\xi) \in \mathscr{E}^{\left(\kappa_{1}\right)}$ and $\sigma(\xi)=1$ for $|\xi| \geq 1$ and $\sigma(\xi)=0$ for $|\xi| \leq$ $1 / 2$.

Definition 1. 1. Let $z^{0}=\left(x^{0}, \xi^{0}\right) \in T^{*} \boldsymbol{R}^{n} \backslash 0$ and $\vartheta \in T_{z^{0}}\left(T^{*} \boldsymbol{R}^{n}\right) \simeq \boldsymbol{R}^{2 n}$. We say that $p(x, \xi)$ (or $p_{m}(x, \xi)$ ) is microhyperbolic with respect to $\vartheta$ at $z^{0}$ if there are a neighborhood $\mathscr{U}$ of $z^{0}$ in $T^{*} \boldsymbol{R}^{n} \backslash 0, l \in \boldsymbol{N} \cup\{0\}$ and positive constants $c$ and $t_{0}$ such that

$$
\left|\sum_{j=0}^{l}(-i t \vartheta)^{j} p_{m}(x, \xi) / j !\right| \geq c t^{l} \quad \text { for }(x, \xi) \in \mathscr{U} \text { and } 0 \leq t \leq t_{0}
$$

where $\vartheta=\left(\vartheta_{x}, \vartheta_{\xi}\right)$ is regarded as a vector field $\vartheta=\vartheta_{x} \cdot(\partial / \partial x)+\vartheta_{\xi} \cdot(\partial / \partial \xi)$.

Remark. (i) The above definition coincides with the definition given in [33]. (ii) When $p_{m}(x, \xi)$ is real analytic, the above definition coincides with the definition of partially microhyperbolicity given by Kashiwara and Kawai [16].

Let $\Omega$ be an open conic set in $T^{*} \boldsymbol{R}^{n} \backslash 0$. We assume that (A-3) $\quad p_{m}(x, \xi)$ is microhyperbolic at each point in $\Omega$.

For $z^{0} \in T^{*} \boldsymbol{R}^{n} \backslash 0$ we can write

$$
p_{m}\left(z^{0}+s \delta z\right)=s^{\mu}\left(p_{m z^{0}}(\delta z)+o(1)\right) \quad \text { as } s \rightarrow 0,
$$

where $p_{m z^{0}}(\delta z) \not \equiv 0$ in $\delta z \in T_{z^{0}}\left(T^{*} R^{n}\right)$, if there are multi-indeces $\alpha$ and $\beta$ such that $p_{m(\beta)}^{(\alpha)}\left(z^{0}\right) \neq 0 . p_{m z^{0}}(\delta z)$ is called the localization polynomial of $p_{m}(z)$ at $z^{0}$ and $\mu \equiv \mu\left(z^{0}\right)$ is called the multiplicity of $p_{m}(z)$ at $z^{0}$. If $p_{m}(z)$ is microhyperbolic with respect to $\vartheta$ at $z^{0}$, then $p_{m z^{0}}(\delta z)$ is hyperbolic with respect to $\vartheta$, i. e.,

$$
p_{m z^{0}}(\delta z-i s \vartheta) \neq 0 \quad \text { for } \delta z \in T_{z^{0}}\left(T^{*} \boldsymbol{R}^{n}\right) \text { and } s>0
$$

(see, e.g., [11]). Therefore, we can define $\Gamma\left(p_{m z}, \vartheta\right)$ as the connected 
component of the set $\left\{\delta z \in T_{z^{0}}\left(T^{*} R^{n}\right) ; p_{m z^{0}}(\delta z) \neq 0\right\}$ which contains $\vartheta$, when $p_{m}(z)$ is microhyperbolic with respect to $\vartheta$ at $z^{0}$. For some properties of hyperbolic polynomials and $\Gamma\left(p_{m z}, \vartheta\right)$ we refer to Atiyah, Bott and Gårding [2].

Definition 1.2. (i) $t(x, \xi) \in C^{1}(\Omega)$ is called a time function for $p_{m}$ in $\Omega$ if $t(x, \xi)$ is real-valued and positively homogeneous of degree 0 in $\xi$, and if $p_{m}(z)$ is microhyperbolic with respect to $-H_{t}(z)$ at every $z \in \Omega$, where $H_{t}(z)=\sum_{j=1}^{n}\left\{\left(\partial t / \partial \xi_{j}\right)(z)\left(\partial / \partial x_{\jmath}\right)-\left(\partial t / \partial x_{j}\right)(z)(\partial /\right.$ $\left.\left.\partial \xi_{,}\right)\right\}$. (ii) Let $t(x, \xi) \in C^{1}(\Omega)$ be a time function for $p_{m}$ in $\Omega$, and let $z \in \Omega$. We define the generalized Hamilton flows $K^{ \pm}(z ; \Omega ; t)$ by

$$
\begin{aligned}
K^{ \pm}(z ; \Omega ; t)= & \{z(s) \in \Omega ; \pm s \geq 0, \text { and }\{z(s)\} \text { is a Lipschitz continuous } \\
& \text { curve in } \Omega \text { satisfying }(d / d s) z(s) \in \Gamma\left(p_{m z(s)},-H_{t}(z(s))\right)^{\circ} \\
& (\text { a.e. } s) \text { and } z(0)=z\},
\end{aligned}
$$

where $\Gamma^{\sigma}=\left\{(\delta x, \delta \xi) \in T_{z}\left(T^{*} R^{n}\right) ; \sigma((\delta y, \delta \eta),(\delta x, \delta \xi))(=\delta x \cdot \delta \eta-\delta y \cdot \delta \xi)\right.$ $\geq 0$ for any $(\delta y, \delta \eta) \in \Gamma\}$ for $z \in T^{*} \boldsymbol{R}^{n} \backslash 0$ and $\Gamma \subset T_{z}\left(T^{*} \boldsymbol{R}^{n}\right)$.

Remark. We should note that Leray [21] and Lascar [20] defined flows similar to $K^{ \pm}(z ; \Omega ; t)$.

Definition 1.3. Let $\kappa>\kappa_{1}$ and $f \in \mathscr{D}^{\left(\kappa_{1}\right)}$. W $W F_{(\kappa)}(f)$ (resp. $W F_{(\kappa)}$ $(f))$ is defined as the complement in $T^{*} \boldsymbol{R}^{n} \backslash 0$ of the collection of all $\left(x^{0}, \xi^{0}\right)$ in $T^{*} \boldsymbol{R}^{n} \backslash 0$ such that there are a neighborhood $U$ of $x^{0}$ and a conic neighborhood $\Gamma$ of $\xi^{0}$ such that for every $\varphi \in \mathscr{D}^{\left(\kappa_{1}\right)}(U)$ and every $A>0$ there is a positive constant $C$ (resp. for every $\varphi \in \mathscr{D}^{\left(\kappa_{1}\right)}$ $(U)$ there are positive constants $A$ and $C$ ) satisfying

$$
|\mathscr{F}[\varphi f](\xi)| \leq C \exp \left[-A|\xi|^{1 / \kappa}\right] \text { for } \xi \in \Gamma,
$$

where $\mathscr{F}[f](\xi) \equiv \hat{f}(\xi)$ denotes the Fourier transform of $f$ (see $[10]$, [28]).

Moreover, we assume that

(A-4) $\quad \mu(\Omega) \equiv \sup _{z \in \Omega} \mu(z)<+\infty$, and $\kappa_{1} \leq \kappa(\Omega) \equiv \min \{2, \mu(\Omega) /(\mu$ $(\Omega)-1)\}$ if $* 1=\left(\kappa_{1}\right)$, and $\kappa_{1}<\kappa(\Omega)$ if $* 1=\left\{\kappa_{1}\right\}$.

Theorem 1.4. Assume that (A-1)-(A-4) are valid, and let $\vartheta: \Omega \ni z$ 
$\mapsto \vartheta(z) \in T_{z}(\Omega)$ be a continuous vector field such that $p_{m}(z)$ is microhyperbolic with respect to $\vartheta(z)$ at each $z \in \Omega$. We denote by $*(\kappa)$ or $\{\kappa\}$, and assume that $\kappa_{1} \leq \kappa \leq \kappa(\Omega)$ and $*=(\kappa)$ when $* 1=\left(\kappa_{1}\right)$ and that $\kappa_{1} \leq \kappa<\kappa(\Omega)$ and $*=\{k\}$ when $* 1=\left\{\kappa_{1}\right\}$. If $u \in \mathscr{D}^{* 1^{\prime}}, z^{0} \in W F_{*}(u) \cap \Omega$ and $W F_{*}(p u) \cap$ $\Omega=\emptyset$, then there are $a \in(-\infty, 0) \cup\{-\infty\}$ and a Lipschitz continuous function $z(t)$ defined on $(a, 0]$ with values in $\Omega$ such that $z(t) \in W F_{*}(u)$ for $t \in(a, 0],(d / d t) z(t) \in \Gamma\left(p_{m z(t)}, \vartheta(z(t))\right)^{\sigma} \cap\{\delta z ;|\delta z|=1\}$ for a.e. $t \in(a, 0]$, and $z(0)=z^{0}$, and $\lim _{t \rightarrow a+0} z(t) \in \partial \Omega$ if $a>-\infty$, where $\partial \Omega$ denotes the boundary of $\Omega$ in $T^{*} \boldsymbol{R}^{n}$.

Theorem 1.5. Assume that (A-1)-(A-4) are valid and that $t(z) \in$ $C^{1}(\Omega)$ is a time function for $p_{m}$ in $\Omega$. Moreover, assume that $\kappa_{1} \leq \kappa \leq \kappa(\Omega)$ and $*=(\kappa)$ when $* 1=\left(\kappa_{1}\right)$ and that $\kappa_{1} \leq \kappa<\kappa(\Omega)$ and $*=\{\kappa\}$ when $* 1=\left\{\kappa_{1}\right\} . \quad$ (i) Let $z^{0} \in \Omega$ and $t_{0} \in \boldsymbol{R}$ satisfy $t_{0} \leq t\left(z^{0}\right)$, and assume that $K^{-}\left(z^{0} ; \Omega ; t\right) \cap\left\{z \in \Omega ; t(z) \geq t_{0}\right\} \Subset \Omega$. Then $z^{0} \notin W F_{*}(u)$ if $u \in \mathscr{D}^{* 1^{\prime}}$, $W F_{*}(p u) \cap K^{-}\left(z^{0} ; \Omega ; t\right) \cap\left\{z \in \Omega ; t(z) \geq t_{0}\right\}=\emptyset$ and $W F_{*}(u) \cap K^{-}\left(z^{0} ; \Omega ; t\right)$ $\cap\left\{z \in \Omega ; t(z)=t_{0}\right\}=\emptyset$. (ii) Furthermore, assume that $K^{-}(z ; \Omega ; t) \cap$ $\left\{z^{1} \in \Omega ; t\left(z^{1}\right) \geq t_{0}\right\} \Subset \Omega$ for every $z \in \Omega$. Then

$$
W F_{*}(u) \cap\left\{z \in \Omega ; t(z) \geq t_{0}\right\} \subset
$$

$\left\{z \in \Omega ; z \in K^{+}(w ; \Omega ; t)\right.$ for some $w \in\left(W F_{*}(p u) \cap\left\{z \in \Omega ; t(z) \geq t_{0}\right\}\right) \cup$ $\left.\left(W F_{*}(u) \cap\left\{z \in \Omega ; t(z)=t_{0}\right\}\right)\right\}$ for $u \in \mathscr{D}^{* 1^{\prime}}$.

Remark. Theorem 1.5 is an immediate consequence of Theorem 1.4. We note that there do not always exist time functions for $p_{m}$ even locally (see Proposition 5.1).

The remainder of this paper is organized as follows. In $\S 2$ we shall give preliminary lemmas on calculus of pseudo-differential operators. In $\S 3$ we shall investigate hypoellipticity in Gevrey classes for operators which satisfy the so-called $(H)$-condition (see [9]). The microlocal Cauchy problem will be studied and microlocal parametrices will be constructed in $\S 4$. We shall give the proof of Theorem 1.4 and some remarks in $\S 5$.

\section{§2. Calculus of Pseudo-Differential Operators}

Using pseudo-differential operators of infinite order, we can reduce 
the problem in Gevrey classes to the problem in the Sobolev spaces and prove Theorem 1.4. In doing so, we must establish calculus of pseudo-differential operators of infinite order. By results in this section (Proposition 2.13 below) we can calculate the symbols of the reduced operators. Throughout this paper we denote by $C_{a, b, \ldots}(A$, $B, \cdots)$ a constant depending on $a, b, \cdots$ and $A, B, \cdots$ which is locally bounded in $A, B, \cdots$. Let $\kappa>1$ and $\varepsilon \in \boldsymbol{R}$, and define

$$
\hat{\mathscr{S}}_{\kappa, \varepsilon}=\left\{v(\xi) \in C^{\infty}\left(\boldsymbol{R}^{n}\right) ; \exp \left[\varepsilon\langle\xi\rangle^{1 / \kappa}\right] v(\xi) \in \mathscr{S}\right\} \text {. }
$$

We say that $v_{j} \rightarrow v$ in $\hat{\mathscr{S}}_{\kappa, \varepsilon}$ as $j \rightarrow \infty$ if $\exp \left[\varepsilon\langle\xi\rangle^{1 / \kappa}\right] v,(\xi) \rightarrow \exp \left[\varepsilon\langle\xi\rangle^{1 / \kappa}\right]$ $v(\xi)$ in $\mathscr{S}$ as $j \rightarrow \infty$. Since $\mathscr{D}$ is dense in $\hat{\mathscr{S}}_{k, \varepsilon}$, it is obvious that the dual space $\hat{\mathscr{S}}_{\kappa, \varepsilon}^{\prime}$ of $\hat{\mathscr{S}}_{k, \varepsilon}$ is identified with $\left\{\exp \left[\varepsilon\langle\xi\rangle^{1 / k}\right] v(\xi) \in \mathscr{D}^{\prime}\right.$; $\left.v \in \mathscr{S}^{\prime}\right\}$. For $\varepsilon \geq 0$ we can define

$$
\mathscr{S}_{\kappa, \varepsilon}=\mathscr{F}^{-1}\left[\hat{\mathscr{S}}_{\kappa, \varepsilon}\right]\left(=\mathscr{F}\left[\hat{\mathscr{S}}_{\kappa, \varepsilon}\right]=\left\{u \in \mathscr{S} ; \exp \left[\varepsilon\langle\xi\rangle^{1 / \kappa} \hat{u}(\xi) \in \mathscr{S}\right\}\right) .\right.
$$

We introduce the topology in $\mathscr{S}_{\kappa, \varepsilon}$ so that $\mathscr{F}: \hat{\mathscr{S}}_{\kappa, \varepsilon} \rightarrow \mathscr{S}_{\kappa, \varepsilon}$ is homeomorphic. Denote by $\mathscr{S}_{k, \varepsilon}^{\prime}$ the dual space of $\mathscr{S}_{\kappa, \varepsilon}$ for $\varepsilon \geq 0$. Then we can define the transposed operators ${ }^{t} \mathscr{F}$ and ${ }^{t} \mathscr{F}^{-1}$ of $\mathscr{F}$ and $\mathscr{F}^{-1}$ which map $\mathscr{S}_{\kappa, \varepsilon}^{\prime}$ and $\hat{\mathscr{S}}_{\kappa, \varepsilon}^{\prime}$ onto $\hat{\mathscr{S}}_{\kappa, \varepsilon}^{\prime}$ and $\mathscr{S}_{\kappa, \varepsilon}^{\prime}$, respectively. Since $\hat{\mathscr{S}}_{\kappa,-\varepsilon} \subset \hat{\mathscr{S}}_{\kappa, \varepsilon}^{\prime}\left(\subset \mathscr{D}^{\prime}\right)$ for $\varepsilon \geq 0$, we can define $\mathscr{\mathscr { S }}_{\kappa,-\varepsilon}={ }^{t} \mathscr{F}^{-1}\left[\hat{\mathscr{S}}_{\kappa,-\varepsilon}\right]$ for $\varepsilon \geq 0$. It is easy to see that $\mathscr{S}_{k,-\varepsilon}^{\prime}=\mathscr{F}\left[\hat{\mathscr{S}}_{k,-\varepsilon}^{\prime}\right]$ is the dual space of $\mathscr{S}_{k,-\varepsilon}, \hat{\mathscr{S}}_{\kappa,-\varepsilon}^{\prime} \subset \mathscr{S}^{\prime} \subset \hat{\mathscr{S}}_{k, \varepsilon}^{\prime}, \subset \mathscr{S}_{k,-\varepsilon}^{\prime} \subset \mathscr{S}^{\prime} \subset \mathscr{S}_{k, \varepsilon}^{\prime}$ for $\varepsilon \geq 0$ and that $\mathscr{F}={ }^{t} \mathscr{F}$ on $\mathscr{S}^{\prime}$. So we write ${ }^{t} \mathscr{F}$ as $\mathscr{F}$. Define

$$
H_{\kappa, \varepsilon}^{m}=\left\{u \in \mathscr{S}_{\kappa,-\varepsilon}^{\prime} ;\langle\xi\rangle^{m} \exp \left[\varepsilon\langle\xi\rangle^{1 / \kappa}\right] \hat{u}(\xi) \in L^{2}\right\}, L_{\kappa, \varepsilon}^{2}=H_{\kappa, \varepsilon}^{0},
$$

where $m \in \mathbb{R}$ and $\varepsilon \in \mathbb{R}$.

Lemma 2.1. (i) $\mathscr{D}^{(x)}$ is a dense subspace of $\mathscr{S}_{x, \varepsilon}$. (ii) $\mathscr{D}^{(k)} \subset \cup_{\varepsilon>0}$

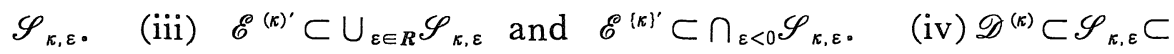
$\mathscr{S}_{\kappa, \varepsilon^{\prime}} \subset H_{\kappa, \varepsilon}^{m} \subset H_{k, \varepsilon^{\prime}}^{m^{\prime}} \subset H_{k, \varepsilon^{\prime}}^{m^{\prime}} \subset \mathscr{S}_{\kappa,-\varepsilon^{*}}^{\prime} \subset \mathscr{D}^{(k)^{\prime}}$, where $\varepsilon \geq \varepsilon^{\prime}>\varepsilon^{\prime \prime}, m \geq m^{\prime}$ and $m^{\prime \prime} \in \boldsymbol{R}$.

Proof. The assertions (ii) and (iii) can be proved by the PaleyWiener theorem in Gevrey classes (see, e.g., [18]). We can also prove that $w_{k}(\xi) \equiv \chi(\xi / k) v(\xi) \rightarrow v(\xi)$ in $\hat{\mathscr{S}}_{k, \varepsilon}$ as $k \rightarrow \infty$ and that $v_{k j}(\xi)$ $\equiv \int \hat{\chi}(\eta) w_{k}(\xi-\eta / j) d \eta \rightarrow w_{k}(\xi)$ in $\hat{\mathscr{S}}_{k, \varepsilon}$ as $j \rightarrow \infty$, where $\chi \in \mathscr{D}^{(r)}, \chi(\xi)$ $=1$ if $|\xi| \leq 1$ and $\chi(\xi)=0$ if $|\xi| \geq 2$, and $d \eta=(2 \pi)^{-n} d \eta$. This proves the assertion (i).

Q. E. D. 
In this paper we shall frequently use the following facts without quoting.

Lemma 2.2. (i) $N ! \leq c e^{-N} N^{N+1 / 2}$ for $N \geq 1$, where $c$ is a positive constant. (ii) For $t \geq 1$

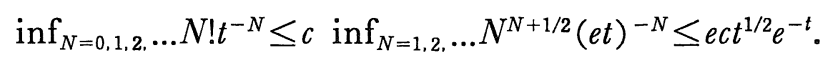

(iii) $|\alpha| ! \leq n^{|\alpha|} \alpha !$ and $\sum_{\mid \beta !=l, \beta \leq \alpha}\left(\begin{array}{c}\alpha \\ \beta\end{array}\right)=\left(\begin{array}{c}|\alpha| \\ l\end{array}\right)$, where $\left(\begin{array}{l}\alpha \\ \beta\end{array}\right)=\alpha ! /(\beta !(\alpha-\beta) !)$.

(iv) $\sum_{: \alpha \mid=N}(\alpha ! / N !)^{\kappa-1} \leq c_{\kappa}^{n-1}$ if $\kappa>1$, where $c_{\kappa}$ is a constant depending only on $\kappa$. (v) $\sum_{k=0}^{\infty} k !^{1-\kappa} t^{k}=c_{\kappa}(t)<+\infty$ if $t>0$ and $\kappa>1$. (vi) $\langle\xi+\eta\rangle_{h} \leq$ $\langle\xi\rangle_{h}+|\eta|$, where $\langle\xi\rangle_{h}=\left(h^{2}+|\xi|^{2}\right)^{1 / 2}$. (vii) $\left|\partial_{\xi}^{\alpha}\langle\xi\rangle_{h}^{1-k}\right| \leq(1+\sqrt{2})^{|\alpha|}(|\alpha|$ $+[k]) !\langle\xi\rangle_{h}^{1-k-|\alpha|} /[k]$ !, where $k \geq 0$ and $[k]$ denotes the largest integer $\leq k$. (viii) Let $1 \leq \kappa^{\prime}<\kappa$ and $N \in \boldsymbol{N} \cup\{0\}$, and assume that $\chi(\xi) \in C^{\infty}\left(\boldsymbol{R}^{n}\right)$ satisfies

$$
\left|\chi^{(\alpha+\beta)}(\xi)\right| \leq\left. C A^{|\alpha|} B^{|\beta|} N^{|\alpha|}|\beta|\right|^{\left.\right|^{\prime}} \text { for }|\alpha| \leq N \text { and any } \beta \text {. }
$$

Then, for any $c>0$, and $d>0$ there is $C_{A, B, c, d}>0$ such that

$$
\left|\partial_{\eta}^{\alpha+\beta} \partial_{\xi}^{\tau} \chi\left(\eta\langle\xi\rangle_{h}^{ \pm 1}\right)\right| \leq C_{A, B, c, d} A^{|\alpha|} d^{|\beta|+|\gamma|} N^{|\alpha|}(|\beta|+|\gamma|) !^{\mid \kappa}\langle\xi\rangle_{h}^{ \pm(|\alpha|+|\beta|)-|\gamma|}
$$

for $|\eta|\langle\xi\rangle_{h}^{+1} \leq c, h>0,|\alpha| \leq N$ and any $\beta$ and $\gamma$.

Proof. The assertions (i)-(iii), (v) and (vi) are obvious. The assertion (iv) can be proved by induction on the dimension $n$. The assertion (vii) can be proved by induction on $|\alpha|$. We note that a similar estimate to (vii) can be also obtained by Cauchy's estimates. In order to prove (viii) it suffices to prove that

$$
\left|\partial_{\eta}^{\alpha+\beta} \partial_{\xi}^{\gamma} \chi\left(\eta\langle\xi\rangle_{h}^{ \pm 1}\right)\right| \leq C A^{|\alpha|} B^{|\beta|} B_{1}^{|\gamma|} N^{|\alpha|} !(|\beta|+|\gamma|) !^{\mid \kappa^{\prime}}\langle\xi\rangle_{h}^{ \pm(|\alpha|+|\beta|)-|\gamma|}
$$

for $|\eta|\langle\xi\rangle_{h}^{ \pm 1} \leq c,|\alpha| \leq N$ and any $\beta$ and $\gamma$, which can be proved by induction on $|\gamma|$. Here $B_{1}$ depends on $A, B$ and $C$. Q.E.D.

Let $p(\xi, y, \eta)$ be a symbol satisfying

$$
\left|\partial_{\xi}^{\alpha} \partial_{\eta}^{\beta} D_{y}^{r} p(\xi, y, \eta)\right| \leq C_{\alpha, \beta} A^{|\gamma|}|\gamma| !^{\kappa} \exp \left[\delta_{1}\langle\xi\rangle^{1 / \kappa}+\delta_{2}\langle\eta\rangle^{1 / \kappa}\right]
$$

for $(\xi, y, \eta) \in \boldsymbol{R}^{n} \times \boldsymbol{R}^{n} \times \boldsymbol{R}^{n}$ and any multi-indeces $\alpha, \beta$ and $\gamma$, where $A>0, \delta_{1}, \delta_{2} \in \boldsymbol{R}$ and the positive constants $C_{\alpha, \beta}$ depend on $\alpha$ and $\beta$. Define

$$
p\left(D_{x}, y, D_{y}\right) u(x)=\mathscr{F}_{\xi}^{-1}\left[\int e^{-i y \cdot \xi}\left(\int e^{i y \cdot \eta} p(\xi, y, \eta) \hat{u}(\eta) d \eta\right) d y\right](x)
$$


for $u \in \mathscr{D}^{(\kappa)}$.

Proposition 2.3. $p\left(D_{x}, y, D_{y}\right)$ maps continuously $\mathscr{S}_{\kappa, \varepsilon_{2}}$ to $\mathscr{S}_{\kappa, \varepsilon_{1}}$ if $\delta_{2}-\kappa(n A)^{-1 / \kappa}<\varepsilon_{2}, \varepsilon_{1} \leq \varepsilon_{2}-\delta_{1}-\delta_{2}$ and $\varepsilon_{1}<\kappa(n A)^{-1 / \kappa}-\delta_{1}$. In particular, $p\left(D_{x}, y, D_{y}\right)$ maps continuously $\mathscr{S}_{\kappa, \varepsilon}$ to $\mathscr{S}_{\kappa, \varepsilon-\delta_{1}-\delta_{2}}$ if $\left|\varepsilon-\delta_{2}\right|<\kappa(n A)^{-1 / \kappa}$.

Proof. Let $u \in \mathscr{D}^{(\kappa)}$ and write

$\langle\xi\rangle^{j} D_{\xi}^{\alpha}\left\{\exp \left[\varepsilon_{1}\langle\xi\rangle^{1 / \kappa}\right] \mathscr{F}\left[p\left(D_{x}, y, D_{y}\right) u(x)\right](\xi)\right\}=\int F(\xi, \eta) d \eta$, where $F(\xi, \eta)=\int e^{-i y \cdot(\xi-\eta)}\langle\xi\rangle^{j} f(\xi, \eta, y) d y, f(\xi, \eta, y)=\sum_{\alpha^{1}+\alpha^{2}=\alpha} \alpha !\left(\alpha^{1 !} \alpha^{2} !\right)^{-1}$ $(-y)^{\alpha^{1}}\langle y\rangle^{-2 N} D_{\xi}^{\alpha^{2}}\left\langle D_{\eta}\right\rangle^{2 N}\left\{\exp \left[\varepsilon_{1}\langle\xi\rangle^{1 / \kappa}\right] p(\xi, y, \eta) \hat{u}(\eta)\right\}$ and $N=[(n+|\alpha|)$ /2] +1 . Then we have

$$
\begin{aligned}
\mid D_{y}^{\beta} f(\xi, \eta, & y)\left|\leq C_{\alpha} A^{|\beta|}\right| \beta \mid !^{\kappa}\langle y\rangle^{-2 N+|\alpha|}\langle\eta\rangle^{-M} \\
& \times \exp \left[\left(\varepsilon_{1}+\delta_{1}\right)\langle\xi\rangle^{1 / \kappa}+\left(\delta_{2}-\varepsilon_{2}\right)\langle\eta\rangle^{1 / \kappa}\right]|u|_{\mathscr{S}_{\kappa, \varepsilon_{2}}, M+2 N},
\end{aligned}
$$

where $|u|_{\mathscr{S}_{\kappa, \xi^{\prime}}}=\sup _{\xi \in R^{n}, j+|\alpha| \leq l}\left|\langle\xi\rangle^{j} D_{\xi}^{\alpha}\left(\exp \left[\varepsilon\langle\xi\rangle^{1 / \kappa}\right] \hat{u}(\xi)\right)\right|$. Since $\langle\xi+\eta\rangle$ $\leq \sqrt{2}\langle\xi\rangle\langle\eta\rangle$, it follows from Lemma 2.2 that

$$
\begin{aligned}
& |F(\xi, \eta)| \leq C_{A^{\prime}, \alpha, j}\langle\eta\rangle^{-M+j} \\
& \quad \times \exp \left[\left(\varepsilon_{1}+\delta_{1}\right)\langle\xi\rangle^{1 / \kappa}+\left(\delta_{2}-\varepsilon_{2}\right)\langle\eta\rangle^{1 / \kappa}-\kappa\left(n A^{\prime}\right)^{-1 / \kappa}|\xi-\eta|^{1 / \kappa}\right] \\
& \quad \times|u|_{\mathscr{S}_{\kappa, \varepsilon}, M+2 N},
\end{aligned}
$$

where $\left.A^{\prime}\right\rangle A$. Noting that $\pm\langle\eta\rangle^{1 / \kappa}-|\xi-\eta|^{1 / \kappa} \leq \pm\langle\xi\rangle^{1 / \kappa}$, we have

$$
\begin{aligned}
& \quad\left|\int F(\xi, \eta) \dot{d} \eta\right| \leq C_{A^{\prime}, \alpha, j}^{\prime} \\
& \quad \times \exp \left[\left(\varepsilon_{1}+\delta_{1}+\max \left\{\delta_{2}-\varepsilon_{2},-\kappa\left(n A^{\prime}\right)^{-1 / \kappa}\right\}\right)\langle\xi\rangle^{1 / \kappa}\right]|u|_{\mathscr{S}_{\kappa, \varepsilon_{2}}, M+2 N}
\end{aligned}
$$

if $A^{\prime}>A, \delta_{2}-\varepsilon_{2}<\kappa(n A)^{-1 / \kappa}$ and $M>j+n$. This proves the proposition.

Q. E. D.

Corollary. $\quad p\left(D_{x}, y, D_{y}\right)$ maps continuously $\mathscr{S}_{\kappa,-\varepsilon_{2}}^{\prime}$ to $\mathscr{S}_{\kappa,-\varepsilon_{1}}^{\prime}$ if $\delta_{2}-$ $\kappa(n A)^{-1 / \kappa}<\varepsilon_{2}, \varepsilon_{1} \leq \varepsilon_{2}-\delta_{1}-\delta_{2}$ and $\varepsilon_{1}<\kappa(n A)^{-1 / \kappa}-\delta_{1}$.

Let $\left\{\varphi_{j .}^{R}(\xi)\right\} \subset \mathscr{E}^{(\kappa)}$ satisfy the following conditions; $0 \leq \varphi_{j}^{R}(\xi) \leq 1$, $\varphi_{j}^{R}(\xi)=1$ if $\langle\xi\rangle_{h}^{1 / \kappa}>2 R j, \varphi_{J}^{R}(\xi)=0$ if $\langle\xi\rangle_{h}^{1 / \kappa}<R j$, and $\left|\varphi_{j}^{R(\alpha)}(\xi)\right| \leq$ $C_{d} d^{|\alpha|}|\alpha|^{\kappa}\langle\xi\rangle_{h}^{-|\alpha|}$ for any $d>0$, where $R>0, j=0,1,2, \ldots$, and $C_{d}$ is a positive constant depending on $d$. For example, $\varphi_{0}^{R}(\xi) \equiv 1$ and $\varphi_{J}^{R}(\xi) \equiv \chi\left(\langle\xi\rangle_{h} /(R j)^{\kappa}\right) \quad(j=1,2, \cdots)$ satisfy the above conditions if $1<\kappa^{\prime}<\kappa, \chi \in \mathscr{E}^{\left(\kappa^{\prime}\right)}\left(\boldsymbol{R}^{1}\right), 0 \leq \chi(t) \leq 1, \chi(t)=1$ if $t \geq 2^{\kappa}$ and $\chi(t)=0$ if 
$t \leq 1$. A simple calculation gives the following

Lemma 2.4. Let $R_{0}>0, \kappa^{\prime}>0$ and $h>0$. If

$$
\left|q_{s(\beta)}^{(\alpha)}(x, \xi)\right| \leq C_{\alpha} A^{|\beta|} B^{j}|\beta| !^{\kappa} j !^{\kappa^{\prime}}\langle\xi\rangle_{h}^{m-|\alpha|-\jmath \kappa^{\prime} / \kappa} \exp \left[\delta\langle\xi\rangle_{h}^{1 / \kappa}\right]
$$

for any $\alpha, \beta, j=0,1,2, \cdots$ and $\langle\xi\rangle_{h}^{1 / k} \geq R_{0} j$, then $q^{R}(x, \xi)=\sum_{j=0}^{\infty} \varphi_{J}^{R}(\xi)$ $q,(x, \xi)$ is well-defined and satisfies

$$
\left|q_{(\beta)}^{R(\alpha)}(x, \xi)\right| \leq C\left(|\alpha|,\left\{G_{\gamma}\right\}_{, \gamma|\leq| \alpha \mid}\right) A^{|\beta|}|\beta| !^{\kappa}\langle\xi\rangle_{h}^{m-|\alpha|} \exp \left[\delta\langle\xi\rangle_{h}^{1 / \kappa}\right]
$$

for $R \geq \max \left(R_{0}, 2 e^{-1} B^{1 / \kappa^{\prime}}\right)$. Moreover, if

$$
\left|q_{j(\beta)}^{(\alpha)}(x, \xi)\right| \leq C A^{|\alpha|+|\beta|} B^{\jmath}(|\alpha|+|\beta|) !^{\kappa} j !^{\kappa^{\prime}}\langle\xi\rangle_{h}^{m-|\alpha|-\jmath \kappa^{\prime} / \kappa} \exp \left[\delta\langle\xi\rangle_{h}^{1 / \kappa}\right]
$$

for any $\alpha, \beta, j=0,1,2, \cdots$ and $\langle\xi\rangle_{h}^{1 / \kappa} \geq R_{0} j$, then

$$
\left|q_{(\beta)}^{R(\alpha)}(x, \xi)\right| \leq C_{A}(C) A^{|\alpha|+|\beta|}(|\alpha|+|\beta|) !^{\kappa}\langle\xi\rangle_{h}^{m-\mid \alpha i} \exp \left[\delta\langle\xi\rangle_{h}^{1 / \kappa}\right]
$$

for $R \geq \max \left(R_{0}, 2 e^{-1} B^{1 / \kappa^{\prime}}\right)$.

Let $h \leq 1$ and $m_{1}, m_{2} \in \boldsymbol{R}$, and let $p(x, \xi, y, \eta)$ be a symbol satisfying

$$
\begin{aligned}
& \left|\partial_{\xi}^{\alpha} D_{x}^{\beta} \partial_{\eta}^{r} D_{y}^{\delta} p(x, \xi, y, \eta)\right|\left(\equiv\left|p_{(\beta)(\delta)}^{(\alpha)(\gamma)}(x, \xi, y, \eta)\right|\right) \\
& \leq L_{i \gamma \mid, A} A^{|\beta|+|\gamma|} A_{1}^{|\alpha|} A_{2}^{|\delta|}|\alpha| !^{\kappa^{\prime}}|\beta| !^{\kappa}|\gamma| !^{\kappa}|\delta| !^{\kappa^{*}} \\
& \quad \times\langle\xi\rangle_{h}^{m_{1}-|\alpha|}\langle\eta\rangle_{h}^{m_{2}-|\gamma|} \exp \left[\delta_{1}\langle\xi\rangle_{h}^{1 / \kappa}+\delta_{2}\langle\eta\rangle_{h}^{1 / \kappa}\right],
\end{aligned}
$$

where $L_{k, A} \equiv C$ or $L_{k, A} A^{k} k !^{k} \equiv C_{k}$. We set $L_{\jmath}=C$ if $L_{k, A} \equiv C$ and $L_{j}=$ $\max _{0 \leq i \leq 1} C_{i}$ if $L_{k, A} A^{k} k !^{k} \equiv C_{k}$. We consider only the cases where " $\kappa^{\prime}=\kappa$ and $\kappa^{\prime \prime}=1 "$ or " $\kappa^{\prime}=1$ and $\kappa^{\prime \prime}=\kappa "$. For $u \in \mathscr{D}^{(\kappa)}$ we can define

$$
p\left(x, D_{x}, y, D_{y}\right) u(x)=\int e^{i x \cdot \xi}\left(\int e^{-i y \cdot \xi}\left(\int e^{i y \cdot \eta} p(x, \xi, y, \eta) \hat{u}(\eta) d \eta\right) d y\right) d \xi
$$

if $\delta_{1}<\kappa\left(n A_{2}\right)^{-1 / \kappa}$ when $\kappa^{\prime}=1$. Here we have applied the same argument as in the proof of Proposition 2.3. Put

$$
q_{j}(x, \xi)=\left.\sum_{|\alpha|=,} \alpha !^{-1} \partial_{\xi}^{\alpha} D_{y}^{\alpha} p(x, \xi, y, \eta)\right|_{y=x, \eta=\xi}, j=0,1,2, \cdots
$$

Then we have

$$
\begin{aligned}
& \left|q_{\jmath(\beta)}^{(\alpha)}(x, \xi)\right| \leq\left(\max _{0 \leq k \leq|\alpha|} L_{k, A}\right)(|\alpha|+|\beta|+j) !^{\kappa}\langle\xi\rangle_{h}^{m_{1}+m_{2}-|\alpha|-\jmath} \\
& \times \exp \left[\left(\delta_{1}+\delta_{2}\right)\langle\xi\rangle_{h}^{1 / \kappa}\right] \sum_{\mu=0}^{|\alpha|} \sum_{\nu=0}^{|\beta|} I(\mu, \nu)\left(n A_{1} A_{2}\right)^{\jmath} A^{|\alpha|-\mu+\nu} A_{1}^{\mu} A_{2}^{|\beta|-\nu},
\end{aligned}
$$

where $I(\mu, \nu)=|\alpha| !|\beta| !(j+\mu) ! !^{\prime} \nu !^{\kappa}(|\alpha|-\mu) !^{\kappa}(|\beta|+j-\nu) !^{\kappa^{*}}\{j ! \mu !(|\alpha|-$ $\left.\mu) ! \nu !(|\beta|-\nu) !(|\alpha|+|\beta|+j) !^{\kappa}\right\}^{-1}$. It is easy to see that 


$$
I(\mu, \nu) \leq\left\{\begin{array}{l}
\left(\begin{array}{c}
|\alpha| \\
\mu
\end{array}\right)^{1-\kappa}(|\beta|-\nu) !^{1-\kappa} \quad \text { if } \kappa^{\prime}=\kappa \\
\left(\begin{array}{c}
|\beta| \\
\nu
\end{array}\right)^{1-\kappa} \mu !^{1-\kappa} \quad \text { if } \kappa^{\prime}=1 .
\end{array}\right.
$$

Applying Lemma 2.2, we have

$$
\begin{aligned}
\left|q_{j(\beta)}^{(\alpha)}(x, \xi)\right| \leq & C(\hat{A} / A)\left(\max _{0 \leq k \leq|\alpha|} L_{k, A}\right) \tilde{A}^{|\alpha|+|\beta|} \tilde{B}^{j}(|\alpha|+|\beta|) !^{\kappa} \\
& \times j !^{\kappa}\langle\xi\rangle_{h}^{m_{1}+m_{2}-|\alpha|-j} \exp \left[\left(\delta_{1} \oplus \delta_{2}\right)\langle\xi\rangle_{h}^{1 / \kappa}\right],
\end{aligned}
$$

where

$$
\begin{aligned}
& \tilde{A}=2^{\kappa} \max \left(A, A_{1}\right) \text { and } \hat{A}=A_{2} \text { if } \kappa^{\prime}=\kappa, \\
& \tilde{A}=2^{\kappa} \max \left(A, A_{2}\right) \text { and } \hat{A}=A_{1} \text { if } \kappa^{\prime}=1,
\end{aligned}
$$

and $\tilde{B}=2^{\kappa} n A_{1} A_{2}$. By Lemma 2.4

$$
q^{R}(x, \xi)=\sum_{j=0}^{\infty} \varphi_{j}^{R}(\xi) q_{j}(x, \xi)
$$

can be defined for $R \geq 4 e^{-1}\left(n A_{1} A_{2}\right)^{1 / \kappa}$ and satisfies

$$
\begin{aligned}
\left|q_{(\beta)}^{R(\alpha)}(x, \xi)\right| \leq & C_{\tilde{A}^{\prime}}\left(|\alpha|, L_{|\alpha|}, \tilde{A}, A^{-1}, \hat{A} / A\right) \tilde{A}^{\prime|\beta|}|\beta| !^{\kappa} \\
& \times\langle\xi\rangle_{h}^{m_{1}+m_{2}-|\alpha|} \exp \left[\left(\delta_{1}+\delta_{2}\right)\langle\xi\rangle_{h}^{1 / \kappa}\right],
\end{aligned}
$$

if $R \geq 4 e^{-1}\left(n A_{1} A_{2}\right)^{1 / \kappa}, \tilde{A}^{\prime}>\tilde{A}$ and $L_{k, A} A^{k} k !^{\kappa} \equiv C_{k}$, and

$$
\begin{aligned}
\left|q_{(\beta)}^{R(\alpha)}(x, \xi)\right| & \leq C_{\tilde{A}}(C, \hat{A} / A) \tilde{A}^{|\alpha|+|\beta|}(|\alpha|+|\beta|) !^{\kappa} \\
& \times\langle\xi\rangle_{h}^{m_{1}+m_{2}-|\alpha|} \exp \left[\left(\delta_{1}+\delta_{2}\right)\langle\xi\rangle_{h}^{1 / \kappa}\right],
\end{aligned}
$$

if $R \geq 4 e^{-1}\left(n A_{1} A_{2}\right)^{1 / k}$ and $L_{k, A} \equiv C$. Therefore, Proposition 2.3 shows that $q^{R}(x, D)$ maps continuously $\mathscr{S}_{\kappa, \varepsilon}$ to $\mathscr{S}_{\kappa, \varepsilon-\delta_{1}-\delta_{2}}$ when $\left|\varepsilon-\delta_{1}-\delta_{2}\right|$ $<\kappa(n \tilde{A})^{-1 / \kappa}$ 。

Lemma 2.5. Let $\chi(x)$ be a function in $\mathscr{D}^{(\kappa)}$ such that $0 \leq \chi(x) \leq 1$ and $\chi(x)=1$ near the origin. Then,

$$
\begin{aligned}
& \sigma\left(p\left(x, D_{x}, y, D_{y}\right)\right)(x, \xi) \\
& =\lim _{\jmath \rightarrow \infty} \int e^{-i y \cdot \eta} p(x, \xi+\eta, x+y, \xi) \chi(\eta / j) \chi(y / j) d y \overline{d \eta} \\
& \left(=O s-\int e^{-i y \cdot \eta} p(x, \xi+\eta, x+y, \xi) d y d \eta\right)
\end{aligned}
$$

if $\delta_{1}<\kappa\left(n A_{2}\right)^{-1 / \kappa}$ when $\kappa^{\prime \prime}=\kappa$. Here $\sigma\left(p\left(x, D_{x}, y, D_{y}\right)\right)(x, \xi)$ denotes the simplified symbol of $p$, that is, $p\left(x, D_{x}, y, D_{y}\right) u(x)=\sigma\left(p\left(x, D_{x}, y, D_{y}\right)\right)$ $(x, D) u(x)$ for $u \in \mathscr{D}^{(\kappa)}$.

Proof. Assume that $\delta_{1}<\kappa\left(n A_{2}\right)^{-1 / \kappa}$ when $\kappa^{\prime \prime}=\kappa$. By the same argument as in the proof of Proposition 2.3, we have 


$$
p\left(x, D_{x}, y, D_{y}\right) u(x)=\int G(x, \eta) d \eta \quad \text { for } u \in \mathscr{D}^{(\kappa)}
$$

where $G(x, \eta)=\int\left(\int e^{i x \cdot \xi} e^{i(x-y) \cdot(\eta-\xi)} p(x, \eta, y, \xi) \hat{u}(\xi) d \xi\right) d y$ is integrable in $\eta$. And we have also

$$
G(x, \eta)=\lim _{ر \rightarrow \infty} G_{j}(x, \eta),
$$

where $G_{j}(x, \eta)=\int e^{i x \cdot \xi} e^{i(x-y) \cdot(\eta-\xi)} \chi((\eta-\xi) / j) \chi((y-x) / j) p(x, \eta, y, \xi) \times$ $\hat{u}(\xi) d \xi d y$. Moreover, from the same argument as in the proof of Proposition 2.3, it follows that there is a function $F(x, \eta)$ integrable in $\eta$ satisfying $\left|G_{j}(x, \eta)\right| \leq F(x, \eta)(j=1,2, \cdots)$. Therefore, applying Lebesgue's theorem and Fubini's theorem, we have

$$
\begin{aligned}
& p\left(x, D_{x}, y, D_{y}\right) u(x)=\lim _{j \rightarrow \infty} \int e^{i x \cdot \xi}\left(\int e^{-i y \cdot \eta}\right. \\
& \quad \times p(x, \xi+\eta, x+y, \xi) \chi(\eta / j) \chi(y / j) d y d \eta) \hat{u}(\xi) d \xi .
\end{aligned}
$$

Similarly, there is a function $F_{1}(x, \xi)$ integrable in $\xi$ such that

$$
\begin{aligned}
& \left|\hat{u}(\xi) \int e^{-i y \cdot \eta} p(x, \xi+\eta, x+y, \xi) \chi(\eta / j) \chi(y / j) d y d \eta\right| \\
& =\mid \hat{u}(\xi) \int\left(\int e^{-i y \cdot \eta}\langle y\rangle^{-2 M}\left\langle D_{\eta}\right\rangle^{2 M}\{p(x, \xi+\eta, x+y, \xi)\right. \\
& \quad \times \chi(\eta / j) \chi(y / j)\} d y) d \eta \mid \leq F_{1}(x, \xi),
\end{aligned}
$$

where $M=[n / 2]+1$. So we can apply Lebesgue's theorem to (2.7), which proves the lemma.

Q.E.D.

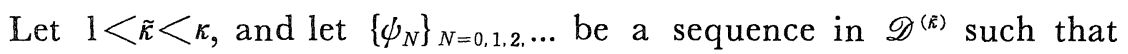
$\psi_{N}(\xi)=1$ if $|\xi| \leq 1 / 4, \phi_{N}(\xi)=0$ if $|\xi| \geq 1 / 2$, and

$$
\left|\psi_{N}^{(\alpha+\beta)}(\xi)\right| \leq C\left(A_{3}(N+1) / 2\right)^{|\alpha|} B^{\mid \beta !}|\beta|^{\tilde{e}} \quad \text { for }|\alpha| \leq N+1 \text {, }
$$

where $A_{3}, B$ and $C$ are positive constants. By Lemma 2.2, for any $d>0$ there is $C_{d}>0$ such that

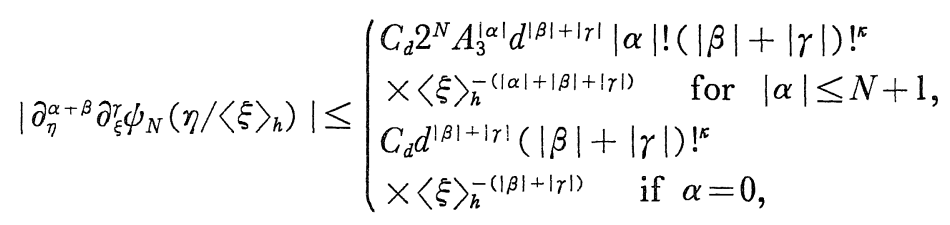

since $(N+1)^{i \alpha \mid} \leq(N+|\alpha|) ! / N ! \leq 2^{N+|\alpha|}|\alpha| !$. Define for $R \geq 4 e^{-1}\left(n A_{1} A_{2}\right)^{1 / \kappa}$

$$
\begin{aligned}
r^{R}(x, D)= & p\left(x, D_{x}, y, D_{y}\right)-q^{R}(x, D), \\
r_{1 N}^{R}(x, \xi)= & \left(\varphi_{N}^{R}(\xi)-\varphi_{N+1}^{R}(\xi)\right)\left\{O s-\int_{N}^{-i y \cdot \eta} e^{-i} p(x, \xi+\eta, x+y, \xi)\right. \\
& \left.\times \phi_{N}\left(\eta /\langle\xi\rangle_{h}\right) d y \ddot{d} \eta-\sum_{k=0}^{N} q_{k}(x, \xi)\right\},
\end{aligned}
$$




$$
\begin{aligned}
r_{2 N}^{R}(x, \xi)= & \left(\varphi_{N}^{R}(\xi)-\varphi_{N+1}^{R}(\xi)\right)\left\{O s-\int_{0} e^{-i y \cdot \eta} p(x, \xi+\eta, x+y, \xi)\right. \\
& \left.\times\left(1-\phi_{N}\left(\eta /\langle\xi\rangle_{h}\right)\right) d y d \eta\right\}
\end{aligned}
$$

Then it is obvious that

$$
r^{R}(x, \xi) \quad\left(=\sigma\left(r^{R}(x, D)\right)(x, \xi)\right)=\sum_{N=0}^{\infty}\left\{r_{1 N}^{R}(x, \xi)+r_{2 N}^{R}(x, \xi)\right\} .
$$

First consider $r_{1 N}^{R}(x, \xi)$. We can write

$$
\begin{aligned}
r_{1 N}^{R}(x, \xi)= & \left(\varphi_{N}^{R}(\xi)-\varphi_{N+1}^{R}(\xi)\right) \int_{0}^{1} \sum_{|\gamma|=N+1}(N+1) \gamma !^{-1}(1-\theta)^{N} \\
& \times\left(\int e^{-i y \cdot \eta}\langle y\rangle^{-2 M} r_{1 N}^{\tau}(x, \xi, \theta y, \eta) d y d \eta\right) d \theta,
\end{aligned}
$$

where $r_{1 N}^{\gamma}(x, \xi, \theta y, \eta)=\left\langle D_{\eta}\right\rangle^{2 M} \partial_{\eta}^{r}\left\{\phi_{N}\left(\eta /\langle\xi\rangle_{h}\right)\left(D_{y}^{r} p\right)(x, \xi+\eta, x+\theta y, \xi)\right\}$ and $M=[n / 2]+1$.

\section{Lemma 2.6。Put}

$$
\begin{gathered}
\delta= \begin{cases}(3 / 2)^{1 / \kappa} \delta_{1}+\delta_{2} & \text { if } \delta_{1} \geq 0, \\
2^{-1 / \kappa} \delta_{1}+\delta_{2} & \text { if } \delta_{1}<0,\end{cases} \\
A^{\prime}= \begin{cases}A & \text { if } \kappa^{\prime}=\kappa, \\
\max \left(A, A_{2}\right) & \text { if } \kappa^{\prime}=1,\end{cases} \\
\hat{A}_{1}= \begin{cases}A_{1} & \text { if } \kappa^{\prime}=\kappa, \\
\max \left(A_{1}, A_{3} / 3\right) & \text { if } \kappa^{\prime}=1 .\end{cases}
\end{gathered}
$$

Then,

(2.11) $\left|r_{1 N(\beta)}^{R(\alpha)}(x, \xi)\right| \leq C\left(|\alpha|, L_{|\alpha|}, A, A_{1}, A_{2} / A, A_{3} / A_{1}\right)\left(2^{\kappa} A^{\prime}\right)^{|\beta|}$

$$
\times|\beta| !^{\kappa}\langle\xi\rangle_{h}^{m_{1}+m_{2}-|\alpha|+n} \exp \left[(\delta-\kappa /(2 R))\langle\xi\rangle_{h}^{1 / \kappa}\right](N+1)^{\kappa / 2} \rho^{N+1},
$$

where $\rho=7 \cdot 2^{\kappa} n \hat{A}_{1} A_{2} R^{-\kappa}$.

Proof. For $|\gamma|=N+1$ we have

$$
\begin{aligned}
\mid \partial_{\xi}^{\alpha} D_{x}^{\beta} r_{1 N}^{\gamma} & (x, \xi, \theta y, \eta) \mid \leq 2^{N} C\left(|\alpha|, L_{|\alpha|}, A, A_{1}\right)(N+1) !(N+1+|\beta|) !^{\kappa} \\
& \times\langle\xi\rangle_{h}^{m_{1}+m_{2}-|\alpha|-N-1} \exp \left[\delta\langle\xi\rangle_{h}^{1 / \kappa}\right] \\
& \times \sum_{\mu=0}^{|\beta|} \sum_{\nu=0}^{N+1} I(\mu, \nu) A^{\mu}\left(3 A_{1}\right)^{\nu} A_{2}^{N+1+|\beta|-\mu} A_{3}^{N+1-\nu}
\end{aligned}
$$

where $I(\mu, \nu)=|\beta| ! \nu !^{\kappa^{\prime}} \mu !^{\kappa}(N+1+|\beta|-\mu) !^{\kappa^{*}}\{\mu !(|\beta|-\mu) ! \nu !(N+1+$ $\left.|\beta|) !^{\kappa}\right\}^{-1}$. Here we have used the facts that $(j+k) ! \leq C_{\varepsilon}(j)(1+\varepsilon)^{k} \times k$ ! for $\varepsilon>0$ and that $\langle\xi\rangle_{h} / 2 \leq\langle\xi+\eta\rangle_{h} \leq\langle\xi\rangle_{h}+|\eta| \leq 3\langle\xi\rangle_{h} / 2$ if $\phi_{N}\left(\eta /\langle\xi\rangle_{h}\right)$ $\neq 0$. It is obvious that 


$$
I(\mu, \nu) \leq \begin{cases}(|\beta|-\mu) !^{1-\kappa}(N+1-\nu) !^{1-\kappa} & \text { if } \kappa^{\prime}=\kappa \\
\left(\begin{array}{c}
|\beta| \\
\mu
\end{array}\right)^{1-\kappa} & \text { if } \kappa^{\prime}=1 .\end{cases}
$$

Therefore, applying Lemma 2.2, we have

$$
\begin{aligned}
\left|\partial_{\xi}^{\alpha} D_{x}^{\beta} r_{1 N}^{\gamma}(x, \xi, \theta y, \eta)\right| \leq & C\left(|\alpha|, L_{|\alpha|}, A, A_{1}, A_{2} / A, A_{3} / A_{1}\right) A^{\prime \prime \beta \mid} \\
& \times\left(7 \hat{A}_{1} A_{2}\right)^{N+1}(N+1) !(N+1+|\beta|) !^{\kappa} \\
& \times\langle\xi\rangle_{h}^{m_{1}+m_{2}-|\alpha|-N-1} \exp \left[\delta\langle\xi\rangle_{h}^{1 / \kappa}\right] .
\end{aligned}
$$

This gives

$$
\begin{aligned}
\mid r_{1 N(\beta)}^{R(\alpha)} & (x, \xi) \mid \leq C^{\prime}\left(|\alpha|, L_{|\alpha|}, A, A_{1}, A_{2} / A, A_{3} / A_{1}\right) A^{\prime|\beta|} \\
& \times\left(7 n \hat{A}_{1} A_{2}\right)^{N+1}(N+1+|\beta|) !^{\kappa}\langle\xi\rangle_{h}^{m_{1}+m_{2}-|\alpha|-N-1+n} \\
& \times \exp \left[\delta\langle\xi\rangle_{h}^{1 / \kappa}\right] \Phi_{N}^{R}(\xi),
\end{aligned}
$$

where $\Phi_{N}^{R}(\xi)$ is the characteristic function of $\left\{\xi \in \boldsymbol{R}^{n} ; R N \leq\langle\xi\rangle_{h}^{1 / \kappa} \leq\right.$ $2 R(N+1)\}$. From Lemma 2.2 it follows that

$$
\begin{aligned}
& \left|r_{1 N(\beta)}^{R(\alpha)}(x, \xi)\right| \leq C^{\prime \prime}\left(|\alpha|, L_{|\alpha|}, A, A_{1}, A_{2} / A, A_{3} / A_{1}\right) \\
& \quad \times\left(2^{\kappa} A^{\prime}\right)^{|\beta|}|\beta| !^{\kappa}\langle\xi\rangle_{h}^{m_{1}+m_{2}-|\alpha|+n} \exp \left[(\delta-\tilde{\delta})\langle\xi\rangle_{h}^{1 / \kappa}\right] \\
& \quad \times(1+1 / N)^{(N+1) \kappa}(N+1)^{\kappa / 2}\left(7 \cdot 2^{\kappa} n e^{2 R \delta \delta-\kappa} \hat{A}_{1} A_{2} R^{-\kappa}\right)^{N+1}
\end{aligned}
$$

for $\tilde{\delta} \leq 0$. (2.12) with $\tilde{\delta}=\kappa /(2 R)$ shows (2.11).

Q.E.D.

Lemma 2.6. implies that

$$
\begin{gathered}
\left|\sum_{N=0}^{\infty} r_{1 N(\beta)}^{R(\alpha)}(x, \xi)\right| \leq C\left(|\alpha|, L_{|\alpha|}, A, A_{1}, A_{2} / A, A_{3} / A_{1}\right)\left(2^{\kappa} A^{\prime}\right)^{|\beta|} \\
\times|\beta| !^{\kappa}\langle\xi\rangle_{h}^{m_{1}+m_{2}-|\alpha|+n} \exp \left[(\delta-\kappa /(2 R))\langle\xi\rangle_{h}^{1 / \kappa}\right]
\end{gathered}
$$

for $R \geq 2^{1+3 / \kappa}\left(n \hat{A}_{1} A_{2}\right)^{1 / \kappa}$. Next let us estimate $r_{2 N}^{R}(x, \xi)$. We can write

$$
r_{2 N}^{R}(x, \xi)=\left(\varphi_{N}^{R}(\xi)-\varphi_{N+1}^{R}(\xi)\right) \int\left(\int e^{-\imath y \cdot \eta} r_{2 N}(x, \xi, y, \eta) d y\right) d \eta,
$$

where $r_{2 N}(x, \xi, y, \eta)=\langle y\rangle^{-2 M}\left\langle D_{\eta}\right\rangle^{2 M}\left\{p(x, \xi+\eta, x+y, \xi)\left(1-\psi_{N}\left(\eta /\langle\xi\rangle_{h}\right)\right)\right\}$ and $M=[n / 2]+1$.

Lemma 2.7. Let $A^{\prime}$ be defined in Lemma 2.6, and let $B>0$ if $\kappa^{\prime}=\kappa$ and $B=A_{2}$ if $\kappa^{\prime}=1$. Then,

$$
\begin{aligned}
\mid r_{2 N(\beta)}^{R(\alpha)}(x, \xi) & : \leq C\left(|\alpha|, L_{|\alpha|}, A, A_{1}, A_{2} / A, A_{2} / B, 1 / B\right) \\
\times & \left(2^{\kappa} A^{\prime}\right)^{|\beta|}|\beta| !^{\kappa} \exp \left[\delta^{\prime}\langle\xi\rangle_{h}^{1 / \kappa}\right]\left(4 R^{-2}+1\right)(N+1)^{-2}
\end{aligned}
$$

if $\left|\delta_{1}\right| \leq 2^{-3} \kappa(n B)^{-1 / \kappa}$ and $\delta^{\prime}=\delta_{1}+\delta_{2}-4^{-1-1 / \kappa} \kappa(n B)^{-1 / \kappa}$.

Proof. The same calculation as in the proof of Lemma 2.6 yields 


$$
\begin{aligned}
\left|\partial_{\xi}^{\alpha} D_{x}^{\beta} D_{y}^{\gamma} r_{2 N}(x, \xi, y, \eta)\right| \leq C\left(|\alpha|, L_{|\alpha|}, A, A_{1}, A_{2} / A, A_{2} / B, 1 / B\right) \\
\quad \times A^{\prime|\beta|} B^{|\gamma|}(|\beta|+|\gamma|) !^{\kappa}\langle y\rangle^{-2 M}|\eta|^{\left|m_{1}\right|+\left|m_{2}\right|} \\
\quad \times \exp \left[\delta_{1}\langle\xi+\eta\rangle_{h}^{1 / \kappa}+\delta_{2}\langle\xi\rangle_{h}^{1 / \kappa}\right] \Psi\left(\eta /\langle\xi\rangle_{h}\right),
\end{aligned}
$$

where $\Psi(\xi)$ is the characteristic function of $\left\{\xi \in \mathbb{R}^{n} ;|\xi| \geq 1 / 4\right\}$. Thus we have

$$
\begin{aligned}
& \left|\partial_{\xi}^{\alpha} D_{x}^{\beta} \int e^{-i y \cdot \eta} r_{2 N}(x, \xi, y, \eta) d y\right| \\
& \leq\left. C_{B^{\prime}}\left(|\alpha|, L_{|\alpha|}, A, A_{1}, A_{2} / A, A_{2} / B, 1 / B\right)\left(2^{\kappa} A^{\prime}\right)^{|\beta|}|\beta|\right|^{\kappa}\langle\eta\rangle^{-n-1} \\
& \quad \times \exp \left[-2^{-1} \kappa\left(n B^{\prime}\right)^{-1 / \kappa}|\eta|^{1 / k}+\delta_{1}\langle\xi+\eta\rangle_{h}^{1 / \kappa}+\delta_{2}\langle\xi\rangle_{h}^{1 / \kappa}\right] \\
& \quad \times \Psi\left(\eta /\langle\xi\rangle_{h}\right)\langle\xi\rangle_{h}^{-2 / \kappa} \\
& \leq C_{B^{\prime}}\left(|\alpha|, L_{|\alpha|}, A, A_{1}, A_{2} / A, A_{2} / B, 1 / B\right)\left(2^{\kappa} A^{\prime}\right)^{|\beta|}|\beta| !^{\kappa}\langle\eta\rangle^{-n-1} \\
& \quad \times \exp \left[\delta^{\prime \prime}\langle\xi\rangle_{h}^{1 / \kappa}\right]\langle\xi\rangle_{h}^{-2 / \kappa}
\end{aligned}
$$

if $B^{\prime}>B, \quad\left|\delta_{1}\right| \leq 2^{-1} \kappa\left(n B^{\prime}\right)^{-1 / \kappa}$ and $\delta^{\prime \prime}=\delta_{1}+\delta_{2}+4^{-1 / \kappa}\left(\left|\delta_{1}\right|-2^{-1} \kappa\left(n B^{\prime}\right)^{-1 / \kappa}\right)$, which proves the lemma.

Lemma 2.7. implies that

$$
\begin{aligned}
\left|\sum_{N=0}^{\infty} r_{2 N(\beta)}^{R(\alpha)}(x, \xi)\right| \leq & C\left(|\alpha|, L_{|\alpha|}, A, A_{1}, A_{2} / A, A_{2} / B, 1 / B, 1 / R\right) \\
& \times\left(2^{\kappa} A^{\prime}\right)^{|\beta|}|\beta| !^{\kappa} \exp \left[\delta^{\prime}\langle\xi\rangle_{h}^{1 / \kappa}\right]
\end{aligned}
$$

if $\left|\delta_{1}\right| \leq 2^{-3} \kappa(n B)^{-1 / \kappa}$. So we have the following

Proposition 2.8. Let $p(x, \xi, y, \eta)$ satisfy (2.1). Then there are $r_{0}>0$ and $\delta\left(1 / \hat{A}_{1}, 1 / A_{2}\right)>0$ such that $\delta\left(1 / \hat{A}_{1}, 1 / A_{2}\right)=\delta\left(1 / A_{2}\right) A_{1}^{-1 / \kappa}$ if $\kappa^{\prime}=\kappa, \delta\left(1 / \hat{A}_{1}, 1 / A_{2}\right)=\delta^{\prime}\left(1 / \hat{A}_{1}\right) A_{2}^{-1 / \kappa}$ if $\kappa^{\prime}=1$, and the following estimates hold if $a \geq 1$ and $R=a r_{0} \hat{A}_{1}^{1 / \kappa} A_{2}^{1 / \kappa}$ :

$$
\begin{aligned}
\left|q_{(\beta)}^{R(\alpha)}(x, \xi)\right| \leq & C_{\tilde{A}^{\prime}}\left(|\alpha|, L_{|\alpha|}, \tilde{A}, A^{-1}, \hat{A} / A\right) \tilde{A}^{\prime|\beta|}|\beta| !^{\kappa} \\
& \times\langle\xi\rangle_{h}^{m_{1}+m_{2}-|\alpha|} \exp \left[\left(\delta_{1}+\delta_{2}\right)\langle\xi\rangle_{h}^{1 / \kappa}\right] \\
& \text { if } \tilde{A}^{\prime}>\tilde{A} \text { and } L_{k, A} A^{k} k !^{\kappa} \equiv C_{k}, \\
\left|q_{(\beta)}^{R(\alpha)}(x, \xi)\right| \leq & C_{\tilde{A}}(C, \hat{A} / A) \tilde{A}^{|\alpha|+|\beta|}(|\alpha|+|\beta|) !^{\kappa} \\
& \times\langle\xi\rangle_{h}^{m_{1}+m_{2}-|\alpha|} \exp \left[\left(\delta_{1}+\delta_{2}\right)\langle\xi\rangle_{h}^{1 / \kappa}\right] \\
& \text { if } L_{k, A} \equiv C, \\
\left|r_{(\beta)}^{R(\alpha)}(x, \xi)\right| \leq & C_{A_{1}, A_{2}}\left(|\alpha|, L_{|\alpha|}, A, A_{2} / A\right)\left(2^{\kappa} A^{\prime}\right)^{|\beta|}|\beta| !^{\kappa} \\
& \times \exp \left[\left(\delta_{1}+\delta_{2}-\delta\left(1 / \hat{A}_{1}, 1 / A_{2}\right) / a\right)\langle\xi\rangle_{h}^{1 / \kappa}\right] \\
& \text { if }\left|\delta_{1}\right| \leq \delta\left(1 / \hat{A}_{1}, 1 / A_{2}\right) / a,
\end{aligned}
$$

where $q^{R}(x, \xi)$ is the symbol defined by $(2.2)$ and $(2.4), r^{R}(x, \xi)=\sigma(p$ $\left.\left(x, D_{x}, y, D_{y}\right)\right)(x, \xi)-q^{R}(x, \xi)$, and $\tilde{A}$ and $\hat{A}, \hat{A}_{1}$ and $A^{\prime}$ are defined by (2.3), (2.10) and (2.9), respectively. 
Proof. If, for example, we choose $r_{0}=2^{1+3 / \kappa} n^{1 / \kappa}$ and

$$
\delta\left(1 / \hat{A}_{1}, 1 / A_{2}\right)=\left\{\begin{array}{l}
2^{-3-3 / \kappa} \kappa\left(n \hat{A}_{1} A_{2}\right)^{-1 / \kappa} \text { when } \kappa^{\prime}=\kappa, \\
2^{-3-3 \kappa} \kappa\left(n A_{2}\right)^{-1 / \kappa} \min \left(\hat{A}_{1}^{-1 / \kappa}, 2^{1 / \kappa}\right) \text { when } \kappa^{\prime}=1,
\end{array}\right.
$$

then the proposition easily follows from (2.5), (2.6) and Lemmas 2. 6 and 2.7.

Q. E. D.

Let $\Lambda(x, \xi)$ be a symbol satisfying

$$
\left|\Lambda_{(\beta)}^{(\alpha)}(x, \xi)\right| \leq C_{0} A_{0}^{|\alpha|+|\beta|}(|\alpha|+|\beta|) !\langle\xi\rangle_{h}^{1 / k-|\alpha|},
$$

and set $\omega_{\beta}^{\alpha}(\Lambda ; x, \xi)=e^{-\Lambda(x, \xi)}\left(e^{\Lambda(x, \xi)}\right)_{(\beta)}^{(\alpha)}$.

Lemma 2.9. If $A_{1}>A_{0}, \rho>0$ and $A_{1} / A_{2}+C_{0} A_{0} \rho^{-1} A_{2}^{-1}\left(1-A_{0} / A_{1}\right)^{-1}$ $\leq 1$, then

$$
\begin{aligned}
\left|\omega_{\beta(\delta)}^{\alpha(\gamma)}(\Lambda ; x, \xi)\right| \leq & A_{1}^{|\gamma|+|\delta|} A_{2}^{|\alpha|+|\beta|}(|\alpha|+|\beta|+|\gamma|+|\delta|) ! \\
& \times\langle\xi\rangle_{h}^{-|\alpha|-|\gamma|} \sum_{k=0}^{|\alpha|+|\beta|} \rho^{k}\langle\xi\rangle_{h}^{k / k} / k ! .
\end{aligned}
$$

In particular, we can take $A_{1}=\left(1+\left(C_{0} / \rho\right)^{1 / 2}\right) A_{0}$ and $A_{2}=\left(1+\left(C_{0} / \rho\right)^{1 / 2}\right)^{2} A_{0}$ for $\rho>0$.

Proof. It is obvious that (2.14) holds for $|\alpha|+|\beta|=0$. Assume that (2. 14) holds for $|\alpha|+|\beta| \leq N$. Let $|\alpha|+|\beta|=N$ and $|e|+\left|e^{\prime}\right|$ $=1$. Then

$$
\begin{aligned}
& \left|\omega_{\beta+e^{\prime}(\delta)}^{\alpha+e(\gamma)}(\Lambda ; x, \xi)\right|= \\
& \left|\omega_{\beta\left(\delta+e^{\prime}\right)}^{\alpha(\gamma+e)}(\Lambda ; x, \xi)+\left(\Lambda_{\left(e^{\prime}\right)}^{(e)}(x, \xi) \omega_{\beta}^{\alpha}(\Lambda ; x, \xi)\right) \underset{(\delta)}{(\gamma)}\right| \\
& \leq A_{1}^{|\gamma|+\mid \delta !} A_{2}^{N+1}(N+|\gamma|+|\delta|+1) !\langle\xi\rangle_{h}^{-|\alpha|-|\gamma|-|e|} \\
& \times \sum_{k=0}^{N+1} \rho^{k}\langle\xi\rangle_{h}^{k / \kappa} / k !\left\{A_{1} / A_{2}+\sum_{\mu=0}^{|\gamma|+i \delta 1}\left(\begin{array}{c}
|\gamma|+|\delta| \\
\mu
\end{array}\right)\right. \\
& \left.\times\left(\begin{array}{c}
N+|\gamma|+|\delta|+1 \\
\mu+1
\end{array}\right)^{-1}(N+1) C_{0} A_{0} \rho^{-1} A_{2}^{-1}\left(A_{0} / A_{1}\right)^{\mu}\right\},
\end{aligned}
$$

which proves the lemma.

Q.E.D.

Corollary. For $\rho>0$,

$$
\begin{aligned}
\left|\left(e^{\Lambda(x, \xi)}\right)_{(\beta)}^{(\alpha)}\right| \leq & \left\{\left(1+\left(C_{0} / \rho\right)^{1 / 2}\right)^{2} A_{0}\right\}^{|\alpha|+|\beta|}(|\alpha|+|\beta|) ! \\
& \times\langle\xi\rangle_{h}^{-|\alpha|} \exp \left[\rho\langle\xi\rangle_{h}^{1 / \kappa}+\operatorname{Re} \Lambda(x, \xi)\right] .
\end{aligned}
$$

Lemma 2.10. Let $p(x, \xi)$ be a symbol satisfying

$$
\left|p_{(\beta)}^{(\alpha)}(x, \xi)\right| \leq L_{|\alpha|, A} A^{|\alpha|+|\beta|}|\alpha| !^{\kappa}|\beta| !^{\kappa}\langle\xi\rangle_{h}^{m-|\alpha|} \exp \left[\delta\langle\xi\rangle_{h}^{1 \mid \kappa}\right],
$$


where $m, \delta \in \boldsymbol{R}$ and $L_{k, A} A^{k} k !^{\kappa} \equiv C_{k} \quad$ or $L_{k, A} \equiv C$, and set $\lambda_{0}=\inf _{L>0}$ $\sup _{x \in \boldsymbol{R}^{n},|\xi| \geq L} \operatorname{Re} \Lambda(x, \xi)\langle\xi\rangle_{h}^{-1 / \kappa}$. Then $\left(e^{\Lambda}\right)(x, D) p(x, D)$ maps continuously $\mathscr{S}_{\kappa, \varepsilon}$ to $\mathscr{S}_{\kappa, \varepsilon-\rho}$ if $\rho>\lambda_{0}+\delta$ and $|\varepsilon-\delta|<\kappa(n A)^{-1 / \kappa}$. Moreover there are $r\left(A_{0}\right)>0$ and $\delta_{A_{0}}>0$ such that $q^{R}(x, \xi)=\sum_{j=0}^{\infty} \varphi_{j}^{R}(\xi) q_{j}(x, \xi)$ is welldefined, and $q^{R}(x, \xi)$ and $r^{R}(x, \xi) \equiv \sigma\left(\left(e^{\Lambda}\right)(x, D) p(x, D)\right)(x, \xi)-q^{R}(x, \xi)$ satisfy the following estimates if $a \geq 1, R=\operatorname{ar}\left(A_{0}\right) A^{1^{\prime \prime} x}$ and $\rho>\lambda_{0}+C_{0}+\delta$, where $q_{j}(x, \xi)=\sum_{|\alpha|=,} \alpha !^{-1} \omega^{\alpha}(\Lambda ; x, \xi) p_{(\alpha)}(x, \xi) e^{\Lambda(x, \xi)}$ :

$$
\begin{aligned}
\left|q_{(\beta)}^{R(\alpha)}(x, \xi)\right| \leq & C_{\rho, A^{\prime}}\left(|\alpha|, L_{|\alpha|}, A, A^{-1}, A_{0} / A\right)\left(2^{\kappa} A^{\prime}\right)^{|\beta|} \\
& \times|\beta| !^{\kappa}\langle\xi\rangle_{h}^{m-|\alpha|} \exp \left[\rho\langle\xi\rangle_{h}^{1 / \kappa}\right] \\
& \text { if } A^{\prime}>A \text { and } L_{K, A} A^{k} k !^{\mid \kappa} \equiv C_{k}, \\
\left|q_{(\beta)}^{R(\alpha)}(x, \xi)\right| \leq & C_{\rho, A}\left(C, A_{0} / A\right)\left(2^{\kappa} A\right)^{|\alpha|+|\beta|}(|\alpha|+|\beta|) !^{\kappa} \\
& \times\langle\xi\rangle_{h}^{m-|\alpha|} \exp \left[\rho\langle\xi\rangle_{h}^{1 / \kappa}\right] \quad \text { if } L_{k, A} \equiv C_{,} \\
\left|r_{(\beta)}^{R(\alpha)}(x, \xi)\right| \leq & C_{\rho, A, A_{0}}\left(|\alpha|, L_{|\alpha|}\right)\left(2^{\kappa} A\right)^{|\beta|} \\
& \times|\beta| !^{\kappa} \exp \left[\left(\rho-a^{-1} \delta_{A_{0}} A^{-1 / \kappa}\right)\langle\xi\rangle_{h}^{1 / \kappa}\right] \\
& \text { if }\left|\lambda_{0}+C_{0}\right|<a^{-1} \delta_{A_{0}} A^{-1 / \kappa}
\end{aligned}
$$

Remark. (i) If $|\varepsilon-\delta|<\kappa(n A)^{-1 / \kappa}$ and $\left|\varepsilon-\lambda_{0}-C_{0}-\delta\right|<2^{-1} \kappa(n A)^{-1 / \kappa}$, then $\left(e^{\Lambda}\right)(x, D) p(x, D)=q^{R}(x, D)+r^{R}(x, D)$ on $\mathscr{S}_{\kappa, \varepsilon}$. (ii) For example, one can take $r\left(A_{0}\right)=2^{1+3 / \kappa}\left(n \hat{A}_{1}\right)^{1 / \kappa}$ and $\delta_{A_{0}}=2^{-3-3 / \kappa} \kappa n^{-1 / \kappa} \min \left(\hat{A}_{1}^{-1 / \kappa}\right.$, $\left.2^{1 / \kappa}\right)$, where $\hat{A_{1}}=\max \left(8 A_{0}, A_{3} / 3\right)$ and $A_{3}$ is the constant in (2.8).

Proof. From the corollary of Lemma 2.9 it follows that

$$
\begin{aligned}
& \mid\left(e^{\Lambda(x, \xi)} p(y, \eta)\right)_{(\beta)}^{(\alpha)(\gamma)} \mid \leq C_{\rho}\left(A_{0} / A\right) L_{|\gamma| . A} A^{|\beta|+|\gamma|} \\
& \quad \times\left(8 A_{0}\right)^{|\alpha|} A^{|\delta|}|\alpha| !|\beta| !^{\kappa}|\gamma| !^{\kappa}|\delta| !^{\kappa}\langle\xi\rangle_{h}^{-|\alpha|}\langle\eta\rangle_{h}^{m-|\gamma|} \\
& \quad \times \exp \left[\rho\langle\xi\rangle_{h}^{1 / \kappa}+\delta\langle\eta\rangle_{h}^{1 / \kappa}\right]
\end{aligned}
$$

if $\rho>\lambda_{0}+C_{0}$. Therefore, the lemma immediately follows from Propositions 2.3 and 2.8 .

Q. E. D.

Lemma 2.11 ([6], [7], [17], [19]). Let $0 \leq \rho<1$ and $m \in \boldsymbol{R}$. Then, for each $s \in \boldsymbol{R}$ there are $C_{s}>0$ and a non-negative integer $\hat{N}_{s}$ such that

$$
\|\left\langle\langle D\rangle_{h}^{s} a(x, D) u\left\|_{L^{2}} \leq G_{s} M\right\|\langle D\rangle_{h}^{s+m} u \|_{L^{2}} \text { for } u \in H^{s+m}\right.
$$

if $\left|a_{(\beta)}^{(\alpha)}(x, \xi)\right| \leq M\langle\xi\rangle_{h}^{m+(|\beta|-|\alpha|) \rho}$ for $(x, \xi) \in T^{*} \boldsymbol{R}^{n}, h \geq 1,|\alpha| \leq \hat{N}_{s}$ and $|\beta| \leq \hat{N}_{s}$, where $H^{s}$ denotes the Sobolev space of order $s$. 
Proof. Make a change of variables: $y=h^{\rho} x$. Taking $\lambda(\xi)=h^{\rho-1}$ $\times\langle\xi\rangle_{h^{1}}-\rho$ as a basic weight function, Theorem 1.6 in Chapter 7 of [19] gives the lemma.

Q. E. D.

Proposition 2.12. There is $\varepsilon_{0}>0$ such that $p(x, D)$ maps continuously $H_{\kappa, \varepsilon}^{s}$ to $H_{\kappa, \varepsilon-\delta}^{s-m}$ if $p(x, \xi)$ satisfies (2.15) and $|\varepsilon-\delta|<\varepsilon_{0} A^{-1 / \kappa}$.

Remark. Proposition 2. 12 was proved in [13] and [24] when $\delta=0$.

Proof. It suffices to show that $\exp \left[(\varepsilon-\delta)\langle D\rangle_{h}^{1 / \kappa}\right] p(x, D) \exp [-\varepsilon$ $\left.\times\langle D\rangle_{h}^{1 / \kappa}\right]$ maps continuously $H^{s}$ to $H^{s-m}$. By Lemma 2.10 and its remark we can write

$$
\exp \left[(\varepsilon-\delta)\langle D\rangle_{h}^{1 / \kappa}\right] p(x, D)=q(x, D)+r(x, D),
$$

where $q(x, \xi)=\sum_{j=0}^{\infty} \varphi_{j}^{R}(\xi) q_{j}(x, \xi), q,(x, \xi)=\sum_{|\alpha|=j} \alpha !^{-1} \omega^{\alpha}(\xi) p_{(\alpha)}(x, \xi)$ $\exp \left[(\varepsilon-\delta)\langle\xi\rangle_{h}^{1 / \kappa}\right], R=2^{1+3 / \kappa}\left(n \hat{A}_{1} A\right)^{1 / \kappa}, \hat{A}_{1}=\max \left(8 A_{0}, A_{3} / 3\right), A_{0}=1+\sqrt{2}$ and $\omega^{\alpha}(\xi) \equiv \omega^{\alpha}\left((\varepsilon-\delta)\langle\xi\rangle_{h}^{1 / \kappa} ; x, \xi\right)$. Moreover, we have

$$
\begin{aligned}
\left|r_{(\beta)}^{(\alpha)}(x, \xi)\right| \leq & C_{\rho, A}\left(|\alpha|, L_{|\alpha|}\right)\left(2^{\kappa} A\right)^{|\beta|}|\beta| !^{\kappa} \\
& \times \exp \left[\left(\rho-2 \varepsilon_{0} A^{-1 / \kappa}\right)\langle\xi\rangle_{h}^{1 / \kappa}\right]
\end{aligned}
$$

if $\rho>\varepsilon+|\varepsilon-\delta|$ and $|\varepsilon-\delta|+\varepsilon-\delta<2 \varepsilon_{0} A^{-1 / \kappa}$, where $\varepsilon_{0}=2^{-4-3 / \kappa} \kappa\left(n \hat{A_{1}}\right)^{-1 / \kappa}$. Therefore, we have

$$
\left|\left(r(x, \xi) \exp \left[-\varepsilon\langle\xi\rangle_{h}^{1 / \kappa}\right]\right)_{(\beta)}^{(\alpha)}\right| \leq C_{A}\left(|\alpha|,|\beta|, L_{|\alpha|}\right)\langle\xi\rangle_{h}^{m-|\alpha|}
$$

if $|\varepsilon-\delta|<\varepsilon_{0} A^{-1 / \kappa}$. On the other hand, a simple calculation yields

$$
\begin{aligned}
\mid & \left.\left(q_{j}(x, \xi) \exp \left[-\varepsilon\langle\xi\rangle_{h}^{1 / \kappa}\right]\right) \begin{array}{c}
(\alpha) \\
\beta
\end{array}\right) \mid \\
\leq & C\left(|\alpha|,|\beta|, L_{|\alpha|}, A\right)\left(n A A_{0}^{\prime}\right)^{\prime} j !^{\kappa}\langle\xi\rangle_{h}^{m-(1-1 / \kappa)|\alpha|-\jmath} \\
& \times \sum_{k=0}^{j} C_{0}^{k}\langle\xi\rangle_{h}^{k / \kappa} / k ! \\
\leq & C^{\prime}\left(|\alpha|,|\beta|, L_{|\alpha|}, A\right)\langle\xi\rangle_{h}^{m-(1-1 / \kappa)|\alpha|} j^{(\kappa-1) / 2}(j+1) \\
& \times\left\{e^{1-\kappa} n A A_{0}^{\prime} R^{1-\kappa} \max \left(C_{0}, R^{-1}\right)\right\}^{j} \text { if }\langle\xi\rangle_{h}^{1 / \kappa} \geq R j,
\end{aligned}
$$

where $C_{0}=|\varepsilon-\delta|$ and $A_{0}^{\prime}=8 A_{0}$. Since

$$
e^{1-\kappa} n A A_{0}^{\prime} R^{1-\kappa} \max \left(C_{0}, R^{-1}\right)<1 \text { when } C_{0}<\varepsilon_{0} A^{-1 / \kappa} \text {, }
$$

we have

$$
\begin{aligned}
& \left|\left(q(x, \xi) \exp \left[-\varepsilon\langle\xi\rangle_{h}^{1 / \kappa}\right]\right)_{(\beta)}^{(\alpha)}\right| \\
& \leq C^{\prime \prime}\left(|\alpha|,|\beta|, L_{|\alpha|}, A\right)\langle\xi\rangle_{h}^{m-(1-1 / \kappa)|\alpha|}
\end{aligned}
$$

if $|\varepsilon-\delta|<\varepsilon_{0} A^{-1 / \kappa}$. Thus (2.19), (2.20) and Lemma 2.11 show that 
$\exp \left[(\varepsilon-\delta)\langle D\rangle_{h}^{1 / \kappa}\right] p(x, D) \exp \left[-\varepsilon\langle D\rangle_{k}^{1 / \kappa}\right]$ maps continuously $H^{s}$ to $H^{s-m}$ if $|\varepsilon-\delta|<\varepsilon_{0} A^{-1 / \kappa}$.

Q.E. D.

Proposition 2.13. Assume that $\Lambda(x, \xi)$ satisfies (2.13) and that $p(x, \xi)$ is a symbol satisfying (2.15) with $L_{k, A} \equiv C$. Then $\left(e^{\Lambda}\right)(x, D) p(x, D)$ ${ }^{R}\left(e^{-\Lambda}\right)(x, D)$ maps continuously $\mathscr{S}_{k, \varepsilon}$ to $\mathscr{S}_{\kappa, \varepsilon-\rho}$ if $\rho>\lambda_{0}+\lambda_{1}+\delta$ and $\mid \varepsilon-\lambda_{1}$ $-\delta \mid<\kappa(n A)^{-1 / \kappa}$, and $H_{\kappa, \varepsilon}^{s}$ to $H_{\kappa, \varepsilon-\rho}^{s-m}$ if $\rho>\lambda_{0}+\lambda_{1}+\delta, \quad\left|\varepsilon-\lambda_{1}-\delta\right|<\varepsilon_{0} A^{-1 / \kappa}$ and $s \in \boldsymbol{R}$, where $\varepsilon_{0}$ and $\lambda_{0}$ are the constants defined in Proposition 2.12 and Lemma 2. 10, respectively, and $\lambda_{1}=\inf _{L>0} \sup _{x \in R^{n},|\xi| \geq L}-\operatorname{Re} \Lambda(x, \xi)$ $\langle\xi\rangle_{h}^{-1 / \kappa}$. Here ${ }^{R}\left(e^{-\Lambda}\right)(x, D)$ denotes the transposed operator of $\left(e^{-\Lambda}\right)$ $(x,-D)$. Moreover there is $c_{A_{0}}>0$ such that there are symbols $p_{A}(x, \xi)$ and $r_{A}(x, \xi)$ satisfying the following properties if $C_{0}<c_{A_{0}} A^{-1 / \kappa}$ and $|\delta|$ $<c_{A_{0}} A^{-1 / \kappa}$ :

$$
\begin{aligned}
& \left(e^{\Lambda}\right)(x, D) p(x, D)^{R}\left(e^{-\Lambda}\right)(x, D)=p_{\Lambda}(x, D)+r_{\Lambda}(x, D), \\
& \mid\left\{p_{\Lambda}(x, \xi)-\sum_{|\alpha|+|\beta|<N}(\alpha ! \beta !)^{-1}\left(p_{(\beta)}(x, \xi) \omega^{\beta}(\Lambda ; x, \xi)\right.\right.
\end{aligned}
$$$$
\left.\left.\times \omega_{\alpha}(-\Lambda ; x, \xi)\right)^{(\alpha)}\right\}_{(\tilde{\beta})}^{(\alpha)} \mid \leq C_{A, A_{0}}(C, N)\left(2^{\kappa} A\right)^{|\hat{\alpha}|+|\tilde{\beta}|}
$$

$$
\begin{array}{r}
\times(|\tilde{\alpha}|+|\tilde{\beta}|) ! \kappa\langle\xi\rangle_{h}^{m-|\alpha|-(1-1 / \kappa) N} \exp \left[\delta\langle\xi\rangle_{h}^{1 / \kappa}\right], \\
N=0,1,2, \ldots,
\end{array}
$$

(2.22) $\quad\left|r_{\Lambda(\beta)}^{(\alpha)}(x, \xi)\right| \leq\left. C_{A, A_{0}}(|\alpha|, C)\left(2^{3 \kappa} A\right)^{|\beta|}|\beta|\right|^{\kappa}$

$$
\times \exp \left[\left(\delta-c A_{0} A^{-1 / \kappa}\right)\langle\xi\rangle_{h}^{1 / \kappa}\right] \text {, }
$$

$$
\begin{aligned}
r_{\Lambda}: & \mathscr{S}_{\kappa, \varepsilon} \rightarrow \mathscr{S}_{\kappa, \varepsilon-\rho} \text { continuously if } \rho=\delta-c_{A_{0}} A^{-1 / \kappa} \text { and } \\
& |\varepsilon-\delta|<2^{-4} \kappa(n A)^{-1 / \kappa},
\end{aligned}
$$

$$
\begin{gathered}
r_{\Lambda}: H_{\kappa, \varepsilon}^{s} \rightarrow H_{\kappa, \varepsilon-\rho}^{s} \text { continuously if } \rho=\delta-c_{A_{0}} A^{-1 / \kappa}, \\
s, s^{\prime} \in R \text { and }|\varepsilon-\delta|<2^{-4} \varepsilon_{0} A^{-1 / \kappa},
\end{gathered}
$$

Proof. We set $q(x, \xi)=\sum_{j=0}^{\infty} \varphi_{j}^{R}(\xi) q_{j}(x, \xi)$ and $r(x, \xi)=\sigma\left(\left(e^{\Lambda}\right)(x, D)\right.$ $p(x, D))(x, \xi)-q(x, \xi)$, where $R=\operatorname{ar}\left(A_{0}\right) A^{1 / \kappa}, a \geq 1, q_{j}(x, \xi)=\sum_{|\alpha|=j} \alpha !^{-1}$ $\omega^{\alpha}(\Lambda ; x, \xi) p_{(\alpha)}(x, \xi) e^{\Lambda(x, \xi)}$ and $r\left(A_{0}\right)$ is the constant in Lemma 2. 10 . Lemma 2. 10 implies that $q(x, \xi)$ and $r(x, \xi)$ satisfy (2.17) and (2.18), respectively. The symbol $r_{\Lambda}^{\prime}(x, \xi) \equiv \sigma\left(r(x, D)^{R}\left(e^{-\Lambda}\right)(x, D)\right)$ can be written as

$$
r_{\Lambda}^{\prime}(x, \xi)=O s-\int e^{-i y \cdot \eta} r(x, \xi+\eta) e^{-\Lambda(x+y, \xi+\eta)} d y d \eta
$$

if $\left|\lambda_{0}+\lambda_{1}+C_{0}+\delta\right|<a^{-1} \delta_{A_{0}} A^{-1 / \kappa}$ and $\left|\lambda_{0}+C_{0}\right|<a^{-1} \delta_{A_{0}} A^{-1 / \kappa}$, where $\delta_{A_{0}}$ is the constant in Lemma 2.10. Then we have 


$$
\begin{aligned}
& r_{\Lambda(\beta)}^{\prime(\alpha)}(x, \xi)=\int f_{\alpha, \beta}(x, \xi, \eta) d \eta, \\
& f_{\alpha, \beta}(x, \xi, \eta) \\
& =\int e^{-i y \cdot \eta}\langle y\rangle^{-2 M} \partial_{\xi}^{\alpha} D_{x}^{\beta}\left\langle D_{\eta}\right\rangle^{2 M}\left\{r(x, \xi+\eta) e^{-\Lambda(x+y, \xi+\eta)}\right\} d y,
\end{aligned}
$$

where $M=[n / 2]+1$. A simple calculation gives

$$
\begin{aligned}
\left|\eta^{\gamma} f_{\alpha, \beta}(x, \xi, \eta)\right| & \leq C_{A, A_{0}, d}(|\alpha|, C)\left(2^{\kappa} A\right)^{|\beta|}|\beta| !^{\kappa}|\gamma| !^{\kappa} d^{|\gamma|} \\
& \times \exp \left[\left(\delta-c_{A_{0}} A^{-1 / \kappa}\right)\langle\xi+\eta\rangle_{h}^{1 / \kappa}\right]
\end{aligned}
$$

if $d>0,\left|\lambda_{0}+\lambda_{1}+2 C_{0}\right|<a^{-1} \delta_{A_{0}} A^{-1 / \kappa} / 4, c_{A_{0}} \leq a^{-1} \delta_{A_{0}} / 2,\left|\lambda_{0}+\lambda_{1}+C_{0}+\delta\right|<a^{-1}$ $\delta_{A_{0}} A^{-1 / \kappa}$ and $\left|\lambda_{0}+C_{0}\right|<a^{-1} \delta_{A_{0}} A^{-1 / \kappa}$. This gives

$$
\begin{aligned}
\left|f_{\alpha, \beta}(x, \xi, \eta)\right| \leq & C_{A, A_{0}}(|\alpha|, C)\left(2^{\kappa} A\right)^{|\beta|}|\beta| !^{\kappa} \\
& \times \exp \left[\left(\delta-c_{A_{0}} A^{-1 / \kappa}\right)\langle\xi\rangle_{h}^{1 / \kappa}-|\eta|^{1 / \kappa}\right], \\
\left|r_{A(\beta)}^{\prime(\alpha)}(x, \xi)\right| \leq & C_{A, A_{0}}^{\prime}(|\alpha|, C)\left(2^{\kappa} A\right)^{|\beta|}|\beta|^{\kappa} \\
& \times \exp \left[\left(\delta-c_{A_{0}} A^{-1 / \kappa}\right)\langle\xi\rangle_{h}^{1 / \kappa}\right],
\end{aligned}
$$

if $|\delta|<a^{-1} \delta_{A_{0}} A^{-1 / \kappa} / 2, C_{0}<2^{-4} a^{-1} \delta_{A_{0}} A^{-1 / \kappa}$ and $c_{A_{0}} \leq a^{-1} \delta_{A_{0}} / 2$. Put

$$
\begin{aligned}
& p_{\Lambda}(x, \xi)=\sum_{j=0}^{\infty} \varphi_{j}^{R}(\xi) \sum_{|\alpha|=j} \alpha !^{-1}\left\{q(x, \xi)\left(e^{-\Lambda(x, \xi)}\right)_{(\alpha)}\right\}^{(\alpha)}, \\
& r^{\prime \prime}(x, \xi)=\sigma\left(q(x, D)^{R}\left(e^{-\Lambda}\right)(x, D)\right)(x, \xi)-p_{\Lambda}(x, \xi),
\end{aligned}
$$

where $R=\operatorname{ar}\left(A_{0}\right) A^{1 / \kappa}, a \geq a_{0}(\geq 2), a_{0}$ is a constant satisfying $a_{0} r\left(A_{0}\right)$ $\geq 2^{2+3 / \kappa} r_{0} A_{0}^{1 / \kappa}$ and $r_{0}$ is the constant in Proposition 2.8. Then it follows from Proposition 2.8 that

$$
\begin{aligned}
\left|r_{A(\beta)}^{\prime \prime(\alpha)}(x, \xi)\right| \leq & C_{\rho, A, A_{0}}(|\alpha|, C)\left(2^{3 \kappa} A\right)^{|\beta|}|\beta|^{\kappa} \\
& \times \exp \left[\left(\rho-a^{-1} \delta_{A_{0}}^{\prime} A^{-1 / \kappa}\right)\langle\xi\rangle_{h}^{1 / \kappa}\right]
\end{aligned}
$$

if $\rho>\lambda_{0}+\lambda_{1}+2 C_{0}+\delta$ and $\left|\lambda_{0}+\lambda_{1}+2 C_{0}+\delta\right|<a^{-1} \delta_{A_{0}}^{\prime} A^{-1 / \kappa}$, where $\delta_{A_{0}}^{\prime}=$ $2^{-2} a_{0} \delta\left(2^{-3} A_{0}^{-1}\right)$ and $\delta(\cdot)$ is the constant in Proposition 2. 8. In fact, $\mid\left\{q(x, \xi) e^{-\Lambda(y, \xi)} \underset{(\beta)(\gamma)}{(\alpha)} \mid \leq C_{\rho, A}\left(C, A_{0} / A\right)\left(2^{2 \kappa} A\right)^{|\beta|}\right.$ $\times\left(2^{2 \kappa} A\right)^{|\alpha|}\left(8 A_{0}\right)^{|\gamma|}|\alpha| !^{\kappa}|\beta| !^{\kappa}|\gamma| !\langle\xi\rangle_{h}^{m-|\alpha|} \exp \left[\rho\langle\xi\rangle_{h}^{1 / \kappa}\right]$

if $\rho>\lambda_{0}+\lambda_{1}+2 C_{0}+\delta$. (2.25), (2.26) and Propositions 2.3 and 2.12 imply that $r_{\Lambda}(x, \xi) \equiv r_{\Lambda}^{\prime}(x, \xi)+r_{\Lambda}^{\prime \prime}(x, \xi)$ satisfies $(2.22)-(2.24)$ if $c_{A_{0}}$ $\leq \min \left(2^{-4} a^{-1} \delta_{A_{0}}, a^{-1} \delta_{A_{0}}^{\prime} / 6,2^{-4} \kappa n^{-1 / \kappa}, 2^{-4} \varepsilon_{0}\right), C_{0}<c_{A_{0}} A^{-1 / \kappa}, \quad|\delta|<c_{A_{0}} A^{-1 / \kappa}$ and $a \geq a_{0}$. A simple calculation yields

$\left|\sum_{|\alpha|=j,|\beta|=k} g_{\alpha, \beta(\tilde{\beta})}^{(\alpha+\bar{\alpha})}(x, \xi)\right|$

$\leq C\left(C, A_{0} / A\right)\left(8 n A_{0} A\right)^{j+k} A^{|\tilde{\alpha}|+|\tilde{\beta}|}(|\tilde{\alpha}|+|\tilde{\beta}|+j+k) !^{\kappa}\langle\xi\rangle_{h}^{m-|\tilde{\alpha}|-j-k}$ $\times \exp \left[\delta\langle\xi\rangle_{h}^{1 / \kappa}\right]\left(\sum_{l=0}^{j} C_{0}^{l}\langle\xi\rangle_{h}^{l / \kappa} / l !\right)\left(\sum_{l=0}^{k} C_{0}^{l}\langle\xi\rangle_{h}^{l / \kappa} / l !\right)$, 
where $g_{\alpha, \beta}(x, \xi)=(\alpha ! \beta !)^{-1} p_{(\beta)}(x, \xi) \omega^{\beta}(\Lambda ; x, \xi) \omega_{\alpha}(-\Lambda ; x, \xi)$. Here we have used the inequalities that

$$
\begin{aligned}
& \sum_{\mu_{1}+\mu_{2}+\mu_{3}=|\tilde{\alpha}|+j, \nu_{1}+\nu_{2}+\nu_{3}=|\tilde{\beta}|}(|\tilde{\alpha}|+j) !|\tilde{\beta}| ! \mu_{1} !^{\kappa}\left(\nu_{1}+k\right) !^{\kappa} \\
& \quad \times\left(\mu_{2}+\nu_{2}+k\right) !\left(\mu_{3}+\nu_{3}+j\right) !\left\{j ! k ! \mu_{1} ! \mu_{2} ! \mu_{1} ! \nu_{1} ! \nu_{2} ! \nu_{3} !\right. \\
& \left.\quad \times(|\tilde{\alpha}|+|\tilde{\beta}|+j+k) !^{\kappa}\right\}^{-1}\left(2 A_{0} / A\right)^{\mu_{2}+\mu_{3}+\nu_{2}+\nu_{3}} \\
& \leq 2^{j+k} \sum_{\mu_{1}+\mu_{2}+\mu_{3}+\mu_{4}=|\tilde{\alpha}|+|\tilde{\beta}|+j} \mu_{3} !^{1-\kappa} \mu_{4} !^{1-\kappa}\left(\begin{array}{c}
\mu_{1}+\mu_{2} \\
\mu_{1}
\end{array}\right)^{1-\kappa} \\
& \quad \times\left(4 A_{0} / A\right)^{\mu_{3}+\mu_{4} \leq 2^{j+k} C\left(A_{0} / A\right) .}
\end{aligned}
$$

Since $\langle\xi\rangle_{h}^{1 / \kappa} \geq 2(N-1) R$ if $j+k<N, \varphi_{j}^{R}(\xi)=1$ and $\varphi_{k}^{R}(\xi)=1$, we have

$$
\begin{aligned}
& \left|\sum_{|\alpha|+|\beta|<N}\left\{\varphi_{\mid \alpha !}^{R}(\xi)\left(\varphi_{1 \beta \mid}^{R}(\xi) g_{\alpha, \beta}(x, \xi)\right)^{(\alpha)}-g_{\alpha, \beta}^{(\alpha)}(x, \xi)\right\}_{(\tilde{\beta})}^{(\tilde{\alpha})}\right| \\
& \leq C\left(C, A_{0} / A, N, C_{0}, R, A_{0} A\right)\left(2^{\kappa} A\right)^{|\tilde{\alpha}|+\left|\tilde{\beta}_{1}\right|}(|\tilde{\alpha}|+|\tilde{\beta}|) !^{\kappa} \\
& \quad \times\langle\xi\rangle_{h}^{m-|\tilde{\alpha}|-(1-1 / \kappa) N} \exp \left[\delta\langle\xi\rangle_{h}^{1 / \kappa}\right] .
\end{aligned}
$$

Moreover, we have

$$
\begin{aligned}
& \left|\sum_{|\alpha|+|\beta| \geq N}\left\{\varphi_{|\alpha|}^{R}(\xi)\left(\varphi_{|\beta|}^{R}(\xi) g_{\alpha, \beta}(x, \xi)\right)^{(\alpha)}\right\} \begin{array}{c}
(\tilde{\alpha}) \\
(\tilde{\beta})
\end{array}\right| \\
& \leq C\left(C, A_{0} / A\right)\left(2^{\kappa} A\right)^{|\tilde{\alpha}|+|\tilde{\beta}|}(|\tilde{\alpha}|+|\tilde{\beta}|) !^{\mid \kappa}\langle\xi\rangle_{h}^{m-|\tilde{\alpha}|-(1-1 / \kappa) N} \\
& \quad \times \exp \left[\delta\langle\xi\rangle_{h}^{1 / \kappa}\right] \sum_{j=N}^{\infty} \sum_{k=0}^{j}\left(2^{3+\kappa} n A_{0} A\right)^{j} j !^{\kappa}(j R / 2)^{(\kappa-1)(N-j)} \\
& \quad \times\left\{\max \left(C_{0}, 1 / R\right)\right\}^{\prime}(k !(j-k) !)^{-1}(j / 2+1)^{2} \\
& \leq C^{\prime}\left(C, A_{0} / A, N, R\right)\left(2^{\kappa} A\right)^{|\tilde{\alpha}|+|\tilde{\beta}|}(|\tilde{\alpha}|+|\tilde{\beta}|) !^{\kappa} \\
& \quad \times\langle\xi\rangle_{h}^{m-i \tilde{\alpha} \mid-(1-1 / \kappa) N} \exp \left[\delta\langle\xi\rangle_{h}^{1 / \kappa}\right]
\end{aligned}
$$

if $2^{4+2 \kappa} e^{1-\kappa} n A_{0} A R^{1-\kappa} \max \left(C_{0}, 1 / R\right) \leq 1$. Thus we obtain (2.21) if a is chosen large enough and if $c_{A_{0}}$ is chosen small enough.

Q.E.D.

Lemma 2.14. There are symbols $q(x, \xi), \tilde{q}(x, \xi), r(x, \xi)$ and $\tilde{r}(x, \xi)$ such that

$$
\begin{aligned}
& { }^{R}\left(e^{-\Lambda}\right)(x, D)\left(e^{\Lambda}\right)(x, D)=1+q(x, D)+r(x, D), \\
& \left(e^{\Lambda}\right)(x, D)^{R}\left(e^{-\Lambda}\right)(x, D)=1+\tilde{q}(x, D)+\tilde{r}(x, D),
\end{aligned}
$$

where $\sigma(1)(x, \xi)=1$,

$$
\begin{aligned}
& \left|q_{(\beta)}^{(\alpha)}(x, \xi)\right| \leq C_{A_{0}, d}\left(C_{0}\right) d^{|\alpha|+|\beta|}(|\alpha|+|\beta|) !^{\kappa}\langle\xi\rangle_{h}^{1 / \kappa-1-|\alpha|}, \\
& \left|r_{(\beta)}^{(\alpha)}(x, \xi)\right| \leq C_{\rho, A_{0}, d}\left(|\alpha|, C_{0}\right) d^{|\beta|}|\beta| !^{\kappa} \exp \left[-\rho\langle\xi\rangle_{h}^{1 / \kappa}\right]
\end{aligned}
$$

if $d>0$ and $\rho \in \boldsymbol{R}$, and $\tilde{q}(x, \xi)$ and $\tilde{r}(x, \xi)$ satisfy the same estimates as (2.27) and (2.28), respectively. Moreover we have 


$$
\begin{aligned}
& (1+q(x, D)+r(x, D))^{R}\left(e^{-\Lambda}\right)(x, D) \\
= & R\left(e^{-\Lambda}\right)(x, D)(1+\tilde{q}(x, D)+\tilde{r}(x, D)) .
\end{aligned}
$$

Remark. With obvious notations, we have $q(x, \xi) \sim \sum_{\alpha>0} \omega_{(\alpha)}^{\alpha}(-\Lambda$; $x, \xi) / \alpha$ ! and $\tilde{q}(x, \xi) \sim \sum_{\alpha>0} \omega_{\alpha}^{(\alpha)}(-\Lambda ; x, \xi) / \alpha$ !, and we can define $q(x, \xi)$, $\tilde{q}(x, \xi), r(x, \xi)$ and $\tilde{r}(x, \xi)$ as analytic symbols (see [26]).

Proof. From Proposition 2. 8 and Lemmas 2. 4 and 2.9 the lemma easily follows.

Q. E. D.

Lemma 2.15. Assume that a symbol $p(x, \xi . y, \eta)$ satisfies

$$
\begin{aligned}
& \left.\left|p_{(\beta)(\delta)}^{(\alpha)(\gamma)}(x, \xi, y, \eta)\right|\right) \leq C_{\gamma} A^{|\alpha|+|\beta|+|\delta|}|\alpha| !^{\kappa}|\beta| !^{\kappa}|\delta| !^{\kappa} \\
& \quad \times\langle\xi\rangle^{-|\alpha|} \exp \left[\delta_{1}\langle\xi\rangle^{1 / \kappa}+\delta_{2}\langle\eta\rangle^{1 / \kappa}\right] \quad \text { for }(x, \xi),(y, \eta) \in T^{*} \boldsymbol{R}^{n}, \\
& \left|p_{(\beta(\gamma)}^{(\alpha)(\gamma)}(x, \xi+\eta, x+y, \eta)\right| \leq C_{\alpha, \gamma}\left(2^{\kappa} A\right)^{|\beta|+|\delta|}|\beta| !^{\kappa}|\delta| !^{\kappa} \\
& \quad \times \exp \left[-a\langle\xi\rangle^{1 / \kappa}\right] \text { if }|\eta| \leq c_{1}\langle\xi\rangle \text { and }|y| \leq c_{2},
\end{aligned}
$$

where $a \in \boldsymbol{R}, c_{1}$ and $c_{2}$ are positive constants. Then there are $d_{0}>0$ and $d_{1}>0$ such that $p\left(x, D_{x}, y, D_{y}\right)$ maps continuously $\mathscr{S}_{\kappa, \varepsilon}$ to $\mathscr{S}_{\kappa, \varepsilon+\rho}$ for $|\varepsilon+\rho|<\kappa(n A)^{-1 / \kappa} / 2$ and $H_{\kappa, \varepsilon}^{s}$ to $H_{\kappa, \varepsilon+\rho}^{s}$ for $|\varepsilon+\rho|<\varepsilon_{0} A^{-1 / \kappa} / 2$ if $\left|\delta_{1}\right|<d_{0}$ $A^{-1 / \kappa}$ and $\rho=\min \left(a, d_{1} A^{-1 / \kappa}-\delta_{1}-\delta_{2}\right)$, where $\varepsilon_{0}$ is the constant in Proposition 2. 12 .

Proof. By the same argument as in the proof of Lemma 2.5, we have

$$
p\left(x, D_{x}, y, D_{y}\right) u(x)=q(x, D) u(x) \quad \text { for } u \in \mathscr{D}^{(\kappa)}
$$

if $\delta_{1}<\kappa(n A)^{-1 / \kappa}$, where

$$
q(x, \xi)=O s-\int e^{-i y \cdot \eta} p(x, \xi+\eta, x+y, \xi) d y d \eta .
$$

We may assume that $0<c_{1}<1$. Choose $\chi(\xi) \in \mathscr{D}^{(\tilde{\kappa})}(1<\tilde{\kappa}<\kappa)$ so that $\chi(\xi)=1$ for $|\xi| \leq 1 / 2$ and $\chi(\xi)=0$ for $|\xi| \geq 1$. Put

$$
\begin{aligned}
q_{1}(x, \xi)= & \int e^{-i y \cdot \eta} p(x, \xi+\eta, x+y, \xi) \chi\left(\eta /\left(c_{1}\langle\xi\rangle\right)\right) \chi\left(y / c_{2}\right) d y d \eta, \\
q_{2}(x, \xi)= & O s-\int e^{-i y \cdot \eta} p(x, \xi+\eta, x+y, \xi) \chi\left(\eta /\left(c_{1}\langle\xi\rangle\right)\right) \\
& \times\left(1-\chi\left(y / c_{2}\right)\right) d y d \eta \\
q_{3}(x, \xi)= & q(x, \xi)-q_{1}(x, \xi)-q_{2}(x, \xi) .
\end{aligned}
$$

Applying the same arguments as in the proofs of Lemmas 2.6 and 2.7, we have 


$$
\begin{aligned}
& \left|q_{1(\beta)}^{(\alpha)}(x, \xi)\right| \leq C_{\alpha}^{\prime}\left(2^{\kappa} A\right)^{|\beta|}|\beta| !^{\kappa}\langle\xi\rangle^{n} \exp \left[-a\langle\xi\rangle^{1 / \kappa}\right], \\
& \left|q_{2(\beta)}^{(\alpha)}(x, \xi)\right| \leq \inf _{N=0,1,2, \ldots} \mid \int\left(\int e^{-i y \cdot \eta}|y|^{-2 N}\langle y\rangle^{-2 M}\right. \\
& \quad \times\left(y \cdot D_{\eta^{\prime}}\right)^{N} \partial_{\xi}^{\alpha} D_{x}^{\beta}\left\langle D_{\eta}\right\rangle^{2 M}\left\{p(x, \xi+\eta, x+y, \xi) \chi\left(\eta /\left(c_{1}\langle\xi\rangle\right)\right)\right. \\
& \left.\left.\quad \times\left(1-\chi\left(y / c_{2}\right)\right)\right\} d y\right) d \eta \leq C_{\alpha, \rho}(A) A^{|\beta|}|\beta| !^{\kappa} \exp \left[\rho\langle\xi\rangle^{1 / \kappa}\right] \\
& \quad \text { if } \rho>\delta_{1}+\delta_{2}+c_{1}^{1 / \kappa}\left|\delta_{1}\right|-\kappa\left(1-c_{1}\right)^{1 / \kappa} c_{2}^{1 / \kappa}(2 n A)^{-1 / \kappa},
\end{aligned}
$$

where $M=[n / 2]+1$. Similarly, we have

$$
\left|q_{3(\beta)}^{(\alpha)}(x, \xi)\right| \geq C_{\alpha, A, \rho}\left(2^{\kappa} A\right)^{|\beta|}|\beta| !^{\kappa} \exp \left[\rho\langle\xi\rangle^{1 / \kappa}\right]
$$

if $\rho>\delta_{1}+\delta_{2}-\left(c_{1} / 2\right)^{1 / \kappa}\left(\kappa(n A)^{-1 / \kappa} / 2-\left|\delta_{1}\right|\right)$ and $\left|\delta_{1}\right|<\kappa(n A)^{-1 / \kappa} / 2$. In fact,

$$
\begin{aligned}
& \mid \int e^{-i y \cdot \eta}\langle y\rangle^{-2 M} \partial_{\xi}^{\alpha} D_{x}^{\beta}\left\langle D_{\eta}\right\rangle^{2 M} \\
& \quad \times\left\{p(x, \xi+\eta, x+y, \xi)\left(1-\chi\left(\eta /\left(c_{1}\langle\xi\rangle\right)\right)\right)\right\} d y \mid \\
& \geq C_{\alpha, A, \rho}^{\prime}\left(2^{\kappa} A\right)^{|\beta|}|\beta| !^{\kappa} \exp \left[\rho|\eta|^{1 / \kappa}+\delta_{1}\langle\xi+\eta\rangle^{1 / \kappa}+\delta_{2}\langle\xi\rangle^{1 / \kappa}\right] \\
& \quad \times \phi\left(\eta /\left(c_{1}\langle\xi\rangle\right)\right) \quad \text { if } \rho>-\kappa(n A)^{-1 / \kappa} / 2,
\end{aligned}
$$

where $\phi(\xi)$ is the characteristic function of $\{\xi \in R ;|\xi| \geq 1 / 2\}$. Therefore, taking $d_{0}=\min \left(2^{-1-1 / \kappa} \kappa\left\{\left(1-c_{1}\right) c_{2} /\left(n c_{1}\right)\right\}^{1 / \kappa}, \kappa n^{-1 / \kappa} / 4\right)$ and $d_{1}=\min$ $\left(2^{-1-1 / \kappa} \kappa\left\{\left(1-c_{1}\right) c_{2} / n\right\}^{1 / \kappa}, 2^{-2-1 / \kappa} \kappa\left(c_{1} / n\right)^{1 / \kappa}\right)$, the lemma follows from Propositions 2.3 and 2.12 .

Q. E. D.

Corollary 1. Let $1<\kappa_{1} \leq \kappa$, and assume that $p(x, \xi)$ satisfies (A-1). Then we have

$$
W F_{*}(p(x, D) u) \subset W F_{*}(u) \quad \text { for } u \in \mathscr{D}^{* 1^{\prime}},
$$

where $*=(\kappa)$ if $* 1=\left(\kappa_{1}\right)$ and $*=\{\kappa\}$ if $* 1=\left\{\kappa_{1}\right\}$.

Proof. Assume that $\left(x^{0}, \xi^{0}\right) \notin W F_{*}(u)$. Then there are $\chi(x) \in \mathscr{D}^{* 1}$ and $\phi(\xi) \in \mathscr{E}^{* 1}$ such that $\chi(x)=1$ near $x^{0}, \phi(\xi)$ is positively homogeneous of degree 0 for $|\xi| \geq 1, \phi(\xi)=1$ if $|\xi| \geq 1$ and $\xi$ belongs to a conic neighborhood of $\xi^{0}$, and $\phi(D) \chi(x) u \in \mathscr{S}_{\kappa, a}$ for any $a>0$ when $*=(\kappa)$ and for some $a>0$ when $*=\{\kappa\}$. So we have $p(x, D) \psi(D)$ $\times \chi(x) u \in \mathscr{E}^{*}$. Let $\chi_{1}(x) \in \mathscr{D}^{* 1}$ satisfy supp $\chi_{1} \Subset\left\{x \in \boldsymbol{R}^{n} ; \chi(x)=1\right\}$, and let $\phi_{1}(\xi) \in \mathscr{E}^{* 1}$ be a positively homogeneous function of degree 0 for $|\xi| \geq 2$ such that $\operatorname{supp} \phi_{1} \cap\left(\xi \in \boldsymbol{R}^{n} ;|\xi|=2\right\} \Subset\left\{\xi \in \boldsymbol{R}^{n} ; \phi(\xi)=1\right\}$. Then Lemma 2.15 implies that $\chi_{1}(x) p(x, D) \phi(D)(1-\chi(x)) u \in \mathscr{E} *^{* 1}$ and $\phi_{1}(D) \chi_{1}(D) p(x, D)(1-\phi(D)) u \in \mathscr{E}^{* 1}$. Thus we have $\phi_{1}(D) \chi_{1}(x) p(x$, D) $u \in \mathscr{E}^{*}$, which proves $\left(x^{0}, \xi^{0}\right) \notin W F_{*}(p(x, D) u)$.

Q. E. D. 
Corollary 2. Let $\mathscr{C}$ be a conic subset of $T^{*} \boldsymbol{R}^{n} \backslash 0$, and let $\chi(x, \xi) \in$ $\mathscr{E}^{* 1}$ be a positively homogeneous function of degree 0 for $|\xi| \geq 1$ such that $\chi(x, \xi)=1$ near $\mathscr{C} \cap\{|\xi| \geq 1\}$ and $\left\{x \in \boldsymbol{R}^{n} ;(x, \xi) \in \operatorname{supp} \chi\right.$ for some $\left.\xi \in \boldsymbol{R}^{n}\right\}$ is compact. Then, $W F_{*}(u) \cap \mathscr{C}=\varnothing$ if $\chi(x, D) u \in \mathscr{E}^{*}$ and $u \in \mathscr{D}^{* 1}$.

Proof. Let $\chi_{1}(x) \in \mathscr{D}^{* 1}$ and $\phi_{1}(\xi) \in \mathscr{E}^{* 1}$ be functions such that $\chi_{1}(x) \phi_{1}(\xi) \Subset \chi(x, \xi)$, i. e., $\chi_{1}(x) \phi_{1}(\xi)$ is positively homogeneous of degree 0 for $|\xi| \geq M$ and supp $\chi_{1}(x) \phi_{1}(\xi) \cap\{|\xi|=M\} \Subset\{(x, \xi) \in$ $\left.T^{*} \boldsymbol{R}^{n} ; \chi(x, \xi)=1\right\}$ for a sufficiently large $M$. Since $\phi_{1}(D) \chi_{1}(x) u=\psi_{1}$ (D) $\chi_{1}(x) \chi(x, D) u-\psi_{1}(D) \chi_{1}(x)(\chi(x, D)-1) u$ and $\phi_{1}(D) \chi_{1}(x)(\chi(x, D)$ -1) $u \in \mathscr{E}^{* 1}$, we have $\psi_{1}(D) \chi_{1}(x) u \in \mathscr{E}^{*}$ if $\chi u \in \mathscr{E}^{*}$. This proves the lemma.

Q.E. D.

Corollary 3. Let $\mathscr{C}_{1}$ and $\mathscr{C}_{2}$ be conic subsets of $T^{*} \boldsymbol{R}^{n} \backslash 0$ such that $\mathscr{C}_{1} \subset \mathscr{C}_{2}$, i.e., $\mathscr{C}_{1} \cap\{|\xi|=1\} \Subset \mathscr{C}_{2} \cap\{|\xi|=1\}$, and let $\chi(x, \xi) \in \mathscr{E}^{(x)}$ be a function such that $\chi(x, \xi)$ is positively homogeneous of degree 0 in $\xi$ for $|\xi| \geq 1, \chi(x, \xi)=1$ near $\mathscr{C}_{1} \cap\{|\xi| \geq 1\}$ and supp $\chi(x, \xi) \cap\{|\xi| \geq 1\} \Subset$ $\mathscr{C}_{2}$. Assume that symbols $p(x, \xi)$ and $q(x, \xi)$ satisfy

$$
\begin{aligned}
& \left|p_{(\beta)}^{(\alpha)}(x, \xi)\right| \leq\left. C A^{|\alpha|+|\beta|}|\alpha|\right|^{\kappa}|\beta| !^{\kappa} \exp \left[\delta\langle\xi\rangle^{1 / \kappa}\right], \\
& \left|q_{(\beta)}^{(\alpha)}(x, \xi)\right| \leq C^{\prime} A^{|\alpha|+|\beta|}|\alpha| !^{\kappa}|\beta|^{\kappa} \exp \left[\delta^{\prime}\langle\xi\rangle^{1 / \kappa}\right],
\end{aligned}
$$

and supp $q(x, \xi) \cap\{|\xi| \geq 1\}\left(\cap \mathscr{C}_{2} \backslash \mathscr{C}_{1}\right)=\varnothing$. Then there is $\hat{\varepsilon}_{1}>0$ such that $q(x, D)[p(x, D), \chi(x, D)] f \in L_{\kappa, \varepsilon_{1}}^{2}$ for $f \in L_{\kappa, \varepsilon}^{2}$ if $|\varepsilon| \leq \varepsilon_{1} \equiv \hat{\varepsilon}_{1} A^{-1 / \kappa}$ and $|\delta|+\left|\delta^{\prime}\right| \leq \varepsilon_{1}$, where $[A, B]=A B-B A$.

Proof. We can write

$$
q(x, D)[p, \chi]=q(1-\chi) p \chi-q \chi p(1-\chi) .
$$

Let $\chi_{j}(x, \xi) \in \mathscr{E}^{(x)}\left(T^{*} \boldsymbol{R}^{n}\right)(j=1,2)$ satisfy $\chi_{1}(x, \xi) \Subset \chi(x, \xi) \Subset \chi_{2}(x, \xi)$, $\chi_{1}(x, \xi)=1 \quad$ near $\mathscr{C}_{1} \cap\{|\xi| \geq 1\}$ and supp $\chi_{2}(x, \xi) \cap\{|\xi| \geq 1\} \Subset \mathscr{C}_{2}$, and put $q_{1}(x, \xi)=q(x, \xi), \chi_{1}(x, \xi), p_{1}(x, \xi)=p(x, \xi) \chi_{2}(x, \xi), q_{2}(x, \xi)=$ $q(x, \xi)-q_{1}(x, \xi)$ and $p_{2}(x, \xi)=p(x, \xi)-p_{1}(x, \xi)$. Then it follows from Lemma 2.15 that $q_{1}(x, D)(1-\chi(x, D)) f, q_{2}(x, D) \chi(x, D) f$, $q_{2}(x, D) p_{1}(x, D) f$ and $p_{2}(x, D) \chi(x, D) f$ belong to $L_{\kappa, \varepsilon_{1}^{\prime}}^{2}$ if $f \in L_{\kappa, \varepsilon}^{2},|\varepsilon|$ $\leq \varepsilon_{1}^{\prime} \equiv \hat{\varepsilon}_{1}^{\prime} A^{-1 / \kappa}$ and $|\delta|+\left|\delta^{\prime}\right| \leq \varepsilon_{1}^{\prime}$, where $\hat{\varepsilon}_{1}^{\prime}>0$. This proves that $q(1-\chi)$ $p \chi f \in L_{\kappa, \varepsilon_{1}}^{2}$ if $f \in L_{\kappa, \varepsilon}^{2},|\varepsilon| \leq \varepsilon_{1} \equiv \varepsilon_{1} A^{-1 / \kappa}$ and $|\delta|+\left|\delta^{\prime}\right| \leq \varepsilon_{1}$, where $\hat{\varepsilon}_{1}>0$. We 
can also apply the same argument to $q \chi p(1-\chi)$ and prove the assertion.

Q.E. D.

To end this section we have to remark that calculus of pseudodifferential operators in the space of real analytic functions and Gevrey classes has been studied by many authors (see [1], [4], [8], [22-26]).

\section{§3. Hypoellipticity}

To prove Theorem 1.4 we shall prepare several lemmas on construction of parametrices in this section. As a consequence of the lemmas, we shall prove that operators satisfying the so-called $(\mathrm{H})$ condition are hypoelliptic in some Gevrey classes, which was essentially proved by Taniguchi [25].

Let $0<\delta \leq 1-1 / \kappa, 1 / \kappa \leq \rho<1, h \geq 1$ and $m, m^{\prime} \in \boldsymbol{R}$. We say that a symbol $p(x, \xi)$ satisfies the condition $\left(H ; C, A, d_{0}, d_{1}, B, N_{0}\right)$, where $C, A, d_{0}, d_{1}, B>0$ and $N_{0}$ is a non-negative integer, if

$$
\begin{aligned}
& \left|p_{(\beta)}^{(\alpha)}(x, \xi)\right| \leq C A^{|\alpha|+|\beta|}(|\alpha|+|\beta|) !^{\kappa}\langle\xi\rangle_{h}^{m-|\alpha|} \text { for any } \alpha \text { and } \beta, \\
& |p(x, \xi)| \geq d_{0}\langle\xi\rangle_{h}^{m^{\prime}}, \\
& \left|p_{(\beta)}^{(\alpha)}(x, \xi) / p(x, \xi)\right| \leq d_{1} B^{|\alpha|+|\beta|}\langle\xi\rangle_{h}^{\delta|\beta|-\rho|\alpha|} \text { for }|\alpha|,|\beta| \leq N_{0} .
\end{aligned}
$$

Lemma 3.1. Assume that $p(x, \xi)$ satisfies the condition ( $H ; C, A$, $\left.d_{0}, d_{1}, B, N_{0}\right)$ and that $\Lambda(x, \xi)$ satisfies (2.13). Then there are positive constants $\hat{\varepsilon}_{\Lambda}, a_{\Lambda}, h_{p, \Lambda}\left(a, 1 / d_{0}, d_{1}, B, N_{0}\right), c_{d_{1}}, C_{A, A_{0}}(C)$ and $C\left(d_{1}\right)$ and symbols $p_{A}^{a}(x, \xi)$ and $r_{\Lambda}^{a}(x, \xi)$ for $0 \leq a \leq a_{A} A^{-1 / \kappa}$ such that

$$
\begin{aligned}
& \left(e^{a \Lambda}\right)(x, D) p(x, D)^{R}\left(e^{-a \Lambda}\right)(x, D)=p_{\Lambda}^{a}(x, D)+r_{\Lambda}^{a}(x, D), \\
& \left|r_{\Lambda(\beta)}^{a(\alpha)}(x, \xi)\right| \leq C_{A, A_{0}}(|\alpha|, C)\left(2^{4 \kappa} A\right)^{|\beta|}|\beta| !^{k} \exp \left[-3 \varepsilon_{0}\langle\xi\rangle_{h}^{1 / \kappa}\right],
\end{aligned}
$$

$r_{\Lambda}^{a}(x, D)$ maps continuously $L_{\kappa, \varepsilon}^{2}$ to $L_{\kappa, 2 \varepsilon_{0}}^{2}$ if $|\varepsilon| \leq \varepsilon_{0} \equiv \hat{\varepsilon}_{A} A^{-1 / \kappa}$, and $p_{\Lambda}^{a}(x, \xi)$ satisfies the condition $\left(H ; C_{A, A_{0}}(C), 2^{2 \kappa} A, d_{0} / 2, C\left(d_{1}\right), B, N_{0}-r\right)$ if $h \geq h_{p, A}$ $\left(a, 1 / d_{0}, d_{1}, B, N_{0}\right)$ and if $a C_{0} A_{0} B \leq c_{d_{1}}$ when $\delta=1-1 / \kappa$ or $\rho=1 / \kappa$, where $r=\left[\left(m-m^{\prime}+1\right) /(1-1 / \kappa)\right]$. Here $a_{A}$ is a constant depending on $A_{0}$ and $C_{0}$, and $h_{p, \Lambda}(\cdots)$ is a constant depending on $A, C, A_{0}, C_{0}, \cdots$.

Remark. When $\delta=0$ or $\rho=1$, we can also obtain similar results, 
which is not necessary in this paper.

Proof. Applying Proposition 2.13 with $\Lambda(x, \xi)$ replaced by $a \Lambda(x, \xi)$, we obtain $p_{\Lambda}^{a}(x, \xi)$ and $r_{\Lambda}^{a}(x, \xi)$. It is obvious that $r_{\Lambda}^{a}(x, \xi)$ has the properties in the lemma if $\hat{\varepsilon}_{\Lambda}$ and $a_{\Lambda}$ are chosen suitably. $p_{\Lambda}^{a}(x, \xi)$ can be written as

$$
\begin{gathered}
p_{\Lambda}^{a}(x, \xi)=\sum_{|\alpha|+|\beta| \leq r}(\alpha ! \beta !)^{-1}\left\{p_{(\beta)}(x, \xi) \omega^{\beta}(a \Lambda ; x, \xi)\right. \\
\left.\times \omega_{\alpha}(-a \Lambda ; x, \xi)\right\}^{(\alpha)}+\hat{p}_{\Lambda(\beta)}^{a(\alpha)}(x, \xi), \\
\left|\hat{p}_{\Lambda(\beta)}^{a(\alpha)}(x, \xi)\right| \leq C_{A, A_{0}}(C)\left(2^{2 \kappa} A\right)^{|\alpha|+|\beta|}(|\alpha|+|\beta|) !^{\kappa} \\
\times\langle\xi\rangle_{h}^{m-|\alpha|-(1-1 / \kappa)(r+1)} .
\end{gathered}
$$

It is easy to see that

$$
\begin{aligned}
& \left|\left\{\omega^{\tilde{\alpha}}(a \Lambda ; x, \xi)-\left(a \nabla_{\xi} \Lambda(x, \xi)\right)^{\tilde{\alpha}}\right\}_{(\beta)}^{(\alpha)}\right| \\
& \leq C\left(C_{0}, A_{0}, \mathrm{a}, \alpha, \beta, \tilde{\alpha}\right)\langle\xi\rangle_{h}^{-(1-1 / \kappa)|\tilde{\alpha} i-1 / \kappa-| \alpha \mid} \text {, }
\end{aligned}
$$

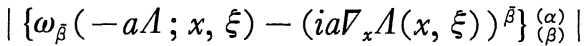

$$
\begin{aligned}
& \leq C\left(C_{0}, A_{0}, a, \alpha, \beta, \tilde{\beta}\right)\langle\xi\rangle_{h}^{|\tilde{\beta}| / \kappa-1 / \kappa-|\alpha|} \text {. }
\end{aligned}
$$

Therefore, we have

$$
\begin{aligned}
\mid & p_{\Lambda(\beta)}^{a(\alpha)}(x, \xi) / p(x, \xi)-p_{(\beta)}^{(\alpha)}(x, \xi) / p(x, \xi) \mid \\
\leqq & \left\{\sum_{f=1}^{r} \sum_{|\tilde{\alpha}|+|\bar{\beta}|=d_{1} B^{j+|\alpha|+|\beta|}\left(a C_{0} A_{0}\right)^{j}}\right. \\
& \times\langle\xi\rangle_{h}^{(\delta+1 / \kappa-1)|\tilde{\beta}|+(1 / \kappa-\rho)|\tilde{\alpha}|} /(\tilde{\alpha} ! \tilde{\beta} !) \\
& \left.+C_{p, \Lambda}\left(a, 1 / d_{0}, d_{1}, B, N_{0}\right)\left(\langle\xi\rangle_{h}^{-1 / \kappa}+\langle\xi\rangle_{h}^{-\delta}+\langle\xi\rangle_{h}^{-1+\rho}\right)\right\} \\
& \times\langle\xi\rangle_{h}^{\delta|\beta|-\rho|\alpha|} \text { if }|\alpha|,|\beta| \leq N_{0}-r .
\end{aligned}
$$

This shows that there are positive constants $h_{p, \Lambda}\left(a, 1 / d_{0}, d_{1}, B, N_{0}\right)$ and $c_{d_{1}}$ such that $\left|p_{\Lambda}^{a}(x, \xi)\right| \geq|p(x, \xi)| / 2$ if $h \geq h_{p, \Lambda}\left(a, 1 / d_{0}, d_{1}, B, N_{0}\right)$ and if $a C_{0} A_{0} B \leq c_{d_{1}}$ when $\delta=1-1 / \kappa$ or $\rho=1 / \kappa$. (3.1) also gives

$$
\left|p_{\Lambda(\beta)}^{a(\alpha)}(x, \xi) / p_{\Lambda}(x, \xi)\right| \leq 2\left(1+d_{1}\right) B^{|\alpha|+|\beta|}\langle\xi\rangle_{h}^{\delta|\beta|-\rho|\alpha|}
$$

if $h \geq h_{p, \Lambda}\left(a, 1 / d_{0}, d_{1}, B, N_{0}\right)$ and $|\alpha|,|\beta| \leq N_{0}-r$, and if $a C_{0} A_{0} B \leq c_{d 1}$ when $\delta=1-1 / \kappa$ or $\rho=1 / \kappa$, modifying $h_{p, \Lambda}\left(a, 1 / d_{0}, d_{1}, B, N_{0}\right)$. This proves the lemma.

Q.E. D.

Lemma 3.2. Assume that $p(x, \xi)$ satisfies the condition ( $H ; C, A$, $\left.d_{0}, d_{1}, B, N_{0}\right)$. Then there is $C\left(d_{1}, N_{0}\right)>0$ such that

$$
\begin{gathered}
\left|(1 / p(x, \xi))_{(\beta)}^{(\alpha)}\right| \leq C\left(d_{1}, N_{0}\right) B^{|\alpha|+|\beta|}\langle\xi\rangle_{h}^{\delta|\beta|-\rho|\alpha|} /|p(x, \xi)| \\
\text { for }|\alpha|,|\beta| \leq N_{0} .
\end{gathered}
$$


Proof. It is sufficient to show that

$$
\begin{array}{rl}
\left|(1 / \hat{\rho}(x, \xi))_{(\beta)}^{(\alpha)}\right| \leq C & C\left(d_{1,}, \alpha|+| \beta \mid\right) B^{|\alpha|+|\beta|} \\
& \times\langle\xi\rangle_{h}^{\delta|\beta|-\rho|\alpha|} /|p(x, \xi)| .
\end{array}
$$

Using the icientity $\left\{p(x, \xi) \partial(1 / p(x, \xi)\}_{(\beta)}^{(\alpha)}=-\{\partial p(x, \xi) / p(x, \xi)\}_{(\beta)}^{(\alpha)}\right.$, $(3.2)$ can be proved by induction on $|\alpha|+|\beta|$.

Q.E.D.

Leming: 3. 3. Assume that $p(x, \xi)$ satisfies the condition $(H ; C, A$, $\left.d_{0}, d_{1}, B, N_{0}\right)$ and that $\delta \leq \rho_{0}$ Put $q(x, \xi)=\sigma(p(x, D) \cdot(1 / p)(x, D))(x, \xi)-1$ and $\tilde{q}(x, \xi)=\sigma((1 / p)(x, D) \circ p(x, D))(x, \xi)-1$. Then

$$
\begin{aligned}
\left|q_{(\beta)}^{(\alpha)}(x, \xi)\right| \leq & \left\{C\left(d_{1}, N_{0}, B^{2} h^{\delta-\rho}\right) B^{2} h^{\delta-\rho} B^{|\alpha|+|\beta|}\right. \\
& \left.+C\left(C, A, 1 / d_{0}, d_{1}, B, N_{0}\right) / h\right\}\langle\xi\rangle_{h}^{\delta|\beta|-\rho|\alpha|}
\end{aligned}
$$

for $|\alpha|,|\beta| \leq N_{0}^{\prime}$ and $N_{0}^{\prime} \leq N_{0}-r-2 L\left(N_{0}^{\prime}\right)-1$, where $r=\left[\left(m-m^{\prime}+n+1\right) /\right.$ $(1-\delta)]$ and $L\left(N_{0}^{\prime}\right)=\left[\left(\rho N_{0}^{\prime}+\delta(r+1)+|m|-m^{\prime}+n+1\right) /(2-2 \delta)\right]+1$, and $\tilde{q}(x, \xi)$ also satisfies the estimates (3.3) for $|\alpha| \leq N_{0}-2[n / 2]-\tilde{r}-3$ and $|\beta| \leq N_{0}-\tilde{r}$, where $\tilde{r}=\left[\left(m-m^{\prime}+1\right) / \rho\right]$.

Proof. Let $\phi \in \mathscr{D}^{\left(\kappa^{\prime}\right)}\left(1<\kappa^{\prime}<\kappa\right)$ be a function such that $\phi(\xi)=1$ for $|\xi| \leq 1 / 4$ and $\phi(\xi)=0$ for $|\xi| \geq 1 / 2$, and write

$$
q(x, \xi)=q_{1}(x, \xi)+\int_{0}^{1}(r+1)(1-\theta)^{r}\left\{q_{2}(x, \xi, \theta)+q_{3}(x, \xi, \theta)\right\} d \theta,
$$

where $q_{1}(x, \xi)=\sum_{1 \leq|\alpha| \leq r} \hat{p}^{(\alpha)}(x, \xi)(1 / p(x, \xi))_{(\alpha)} / \alpha$ ! and $q_{2}(x, \xi, \theta)$ $=\sum_{|\alpha|=r+1} \alpha !^{-1} O s-\int e^{-i y \cdot \eta} p^{(\alpha)}(x, \xi+\eta)(1 / p(x+\theta y, \xi))_{(\alpha)} \phi\left(\eta /\langle\xi\rangle_{h}\right) d y d \eta$. Then we have

$$
\begin{aligned}
\left|q_{1(\beta)}^{(\alpha)}(x, \xi)\right| \leq & C\left(d_{1}, N_{0}, B^{2} h^{\delta-\rho}\right) B^{2} h^{\delta-\rho} B^{|\alpha|+|\beta|}\langle\xi\rangle_{h}^{\delta|\beta|-\rho|\alpha|} \\
& \text { for }|\alpha|,|\beta| \leq N_{0}-r \\
\left|q_{2(\beta)}^{(\alpha)}(x, \xi, \theta)\right| & \leq C\left(C, A, 1 / d_{0}, d_{1}, B, N_{0}\right)\langle\xi\rangle_{h}^{\delta|\beta|-\rho|\alpha|-1} \\
& \text { for }|\alpha| \leq N_{0} \text { and }|\beta| \leq N_{0}-r-1 .
\end{aligned}
$$

A simple calculation yields

$$
\begin{aligned}
& \left|q_{3(\beta)}^{(\alpha)}(x, \xi, \theta)\right| \leq \int\langle\eta\rangle^{-2 L} \sum_{|\gamma|=r+1} \sum_{\alpha^{1}+\alpha^{2}=\alpha, \beta^{1}+\beta^{2}=\beta} \alpha ! \beta ! \\
& \quad \times\left(\gamma ! \alpha^{1} ! \alpha^{2} ! \beta^{1} ! \beta^{2} !\right)^{-1} \mid \partial_{\xi}^{\alpha^{1}} D_{x}^{\beta^{1}}\left\langle D_{\eta}\right\rangle^{2 M}\left\{p^{(r)}(x, \xi+\eta)(1-\right. \\
& \left.\left.\quad \phi\left(\eta /\langle\xi\rangle_{h}\right)\right)\right\}|| \partial_{\xi}^{\alpha^{2}} D_{x}^{r+\beta^{2}}\left\langle D_{y}\right\rangle^{2 L}\left(\langle y\rangle^{-2 M} / p(x+\theta y, \xi)\right) \mid d y d \eta \\
& \quad \leq C\left(C, A, 1 / d_{0}, d_{1}, B, N_{0}\right)\langle\xi\rangle_{h}^{\delta|\beta|-\rho|\alpha|-1}
\end{aligned}
$$

for $|\alpha|,|\beta| \leq N_{0}^{\prime}$ and $N_{0}^{\prime} \leq N_{0}-r-2 L-1$, where $L=L\left(N_{0}^{\prime}\right)$ and $M=$ $[n / 2]+1 .(3.4)-(3.6)$ prove $(3.3)$. Write 


$$
\tilde{q}(x, \xi)=\tilde{q}_{1}(x, \xi)+\int_{0}^{1}(\tilde{r}+1)(1-\theta)^{\xi} \tilde{q}_{2}(x, \xi, \theta) d \theta,
$$

where $\tilde{q}_{1}(x, \xi)=\sum_{1 \leq|\alpha| \leq \tau}(1 / p(x, \xi))^{(\alpha)} p_{(\alpha)}(x, \xi) / \alpha$ !. Then, it is obvious that $\tilde{q}_{1}(x, \xi)$ satisfies the estimates (3.4) for $|\alpha|,|\beta| \leq N_{0}-\tilde{r}$. Moreover, we have

$$
\begin{aligned}
\left|\tilde{q}_{2(\beta)}^{(\alpha)}(x, \xi)\right| \leq & \sum_{|\gamma|=\tilde{\tau}+1} \gamma !^{-1} \int\langle\eta\rangle^{-2 L} \mid \partial_{\xi}^{\alpha} D_{x}^{\beta}\left\langle D_{\eta}\right\rangle^{2 M}\left\langle D_{y}\right\rangle^{2 L} \\
& \times\left\{\langle y\rangle^{-2 M}(1 / p(x, \xi+\eta))^{(\gamma)} p_{(\gamma)}(x+\theta y, \xi)\right\} \mid d y d \eta \\
\leq & C\left(C, A, 1 / d_{0}, d_{1}, B, N_{0}\right)\langle\xi\rangle_{h}^{\delta|\beta|-\rho|\alpha|-1}
\end{aligned}
$$

for $|\alpha| \leq N_{0}-2 M-\tilde{r}-1$ and $|\beta| \leq N_{0}$, where $\tilde{L}=\left[\left\{(\delta+\rho) N_{0}+(1-\rho)\right.\right.$ $\left.\left.n+\left|m^{\prime}\right|\right\} / 2\right]+1$, which proves the lemma.

Q.E.D.

Proposition 3.4. Let $N_{0}$ be a sufficiently large positive integer, and assume that $p(x, \xi)$ satisfies the condition $\left(H ; C, A, d_{0}, d_{1}, B, N_{0}\right)$ and that $\delta \leq \rho$. Then there are positive constants $\hat{\varepsilon}_{0}, h_{p}\left(1 / d_{0}, d_{1}, B\right), c_{d_{1}}$ and $B\left(d_{1}\right)$ and an operator $Q$ such that $Q$ maps continuously $L_{\kappa, \varepsilon}^{2}$ to $H_{\kappa, \varepsilon}^{m^{\prime}}$ and $H_{\kappa, \varepsilon}^{m-m^{\prime}}$ to $H_{\kappa, \varepsilon}^{m}$ and satisfies $Q p(x, D)=I$ on $H_{\kappa, \varepsilon}^{m}$ (or on $L_{\kappa, \varepsilon}^{2}$ ) and $p(x, D) Q=I$ on $H_{\kappa, \varepsilon}^{m-m^{\prime}}\left(\right.$ or on $\left.L_{\kappa, \varepsilon}^{2}\right)$, if $|\varepsilon|<\varepsilon_{0} \equiv \hat{\varepsilon}_{0} A^{-\mathrm{1} / \kappa}$ and $h \geq h_{p}\left(1 / d_{0}, d_{1}, B\right)$, and if $|\varepsilon| B \leq c_{d_{1}}$ when $\delta=1-1 / \kappa$ or $\rho=1 / \kappa$, and if $B \leq B\left(d_{1}\right)$ when $\rho=\delta$. Here $I$ denotes the identity operator, and $h_{p}(\cdots)$ is a constant depending on $A, C, \cdots$. Let $\mathscr{C}_{1}$ and $\mathscr{C}_{2}$ be conic sets in $T^{*} \boldsymbol{R}^{n} \backslash 0$ such that the distance between $\mathscr{C}_{1} \cap\{|\xi|=1\}$ and $\mathscr{C}_{2} \cap\{|\xi|=1\}$ is not less than $d_{2}>0$. If $\chi_{j} \in \mathscr{E}^{(\kappa)}\left(T^{*} \boldsymbol{R}^{n}\right)$, supp $\chi_{j}(x, \xi) \subset \mathscr{C}_{j}$ and $\left|\chi_{j(\beta)}^{(\alpha)}(x, \xi)\right| \leq C_{d} d^{|\alpha|+|\beta|}(|\alpha|$ $+|\beta|) !^{\kappa}\langle\xi\rangle^{-|\alpha|}$ for any $d>0(j=1,2)$, then there are positive constants $\hat{a}_{d_{2}}, h_{p, d_{2}}\left(1 / d_{0}, d_{1}, B\right), c_{d_{1}, d_{2}}$ and $B^{\prime}\left(d_{1}\right)$ such that $\chi_{2}(x, D) Q \chi_{1}(x, D)$ maps continuouslv $L_{\kappa,-a}^{2}$ to $L_{\kappa, a}^{2}$ if $0 \leq a \leq \hat{a}_{d_{2}} A^{-1 / \kappa}$ and $h \geq h_{p, d_{2}}\left(1 / d_{0}, d_{1}, B\right)$, and if $a B \leq c_{d_{1}, d_{2}}$ when $\delta=1-1 / \kappa$ or $\rho=1 / \kappa$, and if $B \leq B^{\prime}\left(d_{1}\right)$ when $\rho=\delta$.

Proof. From Lemma 3.1 with $a \Lambda(x, \xi)$ replaced by $\varepsilon\langle\xi\rangle_{h}^{1 / \kappa}$ it follows that there are $\hat{\varepsilon}_{0}>0$ and symbols $p_{\varepsilon}(x, \xi)$ and $r_{\varepsilon}(x, \xi)$ for $|\varepsilon| \leq \varepsilon_{0} \equiv \hat{\varepsilon}_{0} A^{-1 / \kappa}$ such that

$$
\begin{aligned}
& \exp \left[\varepsilon\langle D\rangle_{h}^{1 / \kappa}\right] p(x, D) \exp \left[-\varepsilon\langle D\rangle_{h}^{1 / \kappa}\right]=p_{\varepsilon}(x, D)+r_{\varepsilon}(x, D), \\
& \left|r_{\varepsilon(\beta)}^{(\alpha)}(x, \xi)\right| \leq C_{A}(|\alpha|,|\beta|, C)\langle\xi\rangle_{h}^{m-|\alpha|} \exp \left[-\varepsilon_{0}\langle\xi\rangle_{h}^{1 / \kappa}\right]
\end{aligned}
$$

if $|\varepsilon| \leq \varepsilon_{0}$, and $p_{\varepsilon}(x, \xi)$ satisfies the condition $\left(H ; C_{A}(C), 2^{2 \kappa} A, d_{0} / 2\right.$, $\left.C\left(d_{1}\right), B, N_{0}-r\right)$, if $\varepsilon$ and $h$ satisfy the following conditions;

$$
|\varepsilon| \leq \varepsilon_{0} \text { and } h \geq h_{p}\left(1 / d_{0}, d_{1}, B\right) \text {, and }|\varepsilon| B \leq c_{d_{1}} \text { when } \delta=1
$$$$
-1 / \kappa \text { or } \rho=1 / \kappa \text {, }
$$ 
where $r=\left[\left(m-m^{\prime}+1\right) /(1-1 / \kappa)\right]$ and $C_{A}(|\alpha|,|\beta|, C), C_{A}(C), C\left(d_{1}\right)$, $h_{p}\left(1 / d_{0}, d_{1}, B\right)$ and $c_{d_{1}}$ are positive constants. We set

$$
q_{\varepsilon}(x, \xi)=\sigma\left(\left(p_{\varepsilon}(x, D)+r_{\varepsilon}(x, D)\right)\left(1 / p_{\varepsilon}\right)(x, D)\right)(x, \xi)-1 .
$$

Applying Lemmas 3.2 and 3.3, we can see that $q_{\varepsilon}(x, \xi)$ satisfies the same estimates as (3.3) if $C\left(C, A, 1 / d_{0}, d_{1}, B, N_{0}\right)$ is replaced by $C_{p}\left(1 / d_{0}, d_{1}, B, N_{0}\right)$ and $\varepsilon$ and $h$ satisfy (3.7) and if $|\alpha|,|\beta| \leq \hat{N}_{m-m^{\prime}}$ and $N_{0} \geq \hat{N}_{m-m^{\prime}}+r+l_{1}+l_{2}$, where $l_{1}=\left[\left(m-m^{\prime}+n+1\right) /(1-\delta)\right], l_{2}=$ $2\left[\left(\rho \hat{N}_{m-m^{\prime}}+\delta\left(l_{1}+1\right)+|m|-m^{\prime}+n+1\right) /(2-2 \delta)\right]+3$ and $\hat{N}_{m-m^{\prime}}$ is the constant in Lemma 2.11. In fact, $\sigma\left(r_{\varepsilon}(x, D)\left(1 / p_{\varepsilon}\right)(x, D)\right)(x, \xi)=O s$ $-\int e^{-i y \cdot \eta} r_{\varepsilon}(x, \xi+\eta) p_{\varepsilon}(x+y, \xi)^{-1} d y d \eta$ can be estimated similarly. Therefore, it follows from Lemma 2.11 that there is the inverse $\left(1+q_{\varepsilon}(x, D)\right)^{-1}$ of $\left(1+q_{\varepsilon}(x, D)\right)$ such that $\left(1+q_{\varepsilon}\right)^{-1}\left(1+q_{\varepsilon}\right)=\left(1+q_{\varepsilon}\right)$ $\left(1+q_{\varepsilon}\right)^{-1}=I$ on $H^{m-m^{\prime}}$ if $\varepsilon$ and $h$ satisfy (3.7), and if $B \leq B\left(d_{1}\right)$ when $\rho=\delta$, where $h_{p}\left(1 / d_{0}, d_{1}, B\right), c_{d_{1}}$ and $B\left(d_{1}\right)$ are suitable positive constants. Put $Q_{\varepsilon}=\exp \left[-\varepsilon\langle D\rangle_{h}^{1 / \kappa}\right]\left(1 / p_{\varepsilon}\right)(x, D)\left(1+q_{\varepsilon}(x, D)\right)^{-1} \exp$ $\left[\varepsilon\langle D\rangle_{h}^{1 / \kappa}\right]$. Then $Q_{\varepsilon}$ maps continuously $H_{\kappa, \varepsilon}^{m-m^{\prime}}$ to $H_{\kappa, \varepsilon}^{m}$ and satisfies $p(x, D) Q_{\varepsilon}=I$ on $H_{\kappa, \varepsilon}^{m-m^{\prime}}$ if $|\varepsilon| \leq \varepsilon_{0}$. Here we have assumed that $N_{0} \geq \hat{N}_{m}+r$, and applied Lemma 2.11 to $\left(1 / p_{\varepsilon}\right)(x, D)$. Put $\tilde{q}_{\varepsilon}(x, \xi)=$ $\sigma\left(\left(1 / p_{\varepsilon}\right)(x, D)\left(p_{\varepsilon}(x, D)+r_{\varepsilon}(x, D)\right)\right)(x, \xi)-1$. Similarly $\left(1+\tilde{q}_{\varepsilon}(x, D)\right)$ has the inverse $\left(1+\tilde{q}_{\varepsilon}(x, D)\right)^{-1}$ on $H^{m^{\prime}}$ if $\varepsilon$ and $h$ satisfy (3.7), and if $B \leq B\left(d_{1}\right)$ when $\rho=\delta$, modifying the constants. If we set $\tilde{Q}_{\varepsilon}=$ $\exp \left[-\varepsilon\langle D\rangle_{h}^{1 / \kappa}\right]\left(1+\tilde{q}_{\varepsilon}(x, D)\right)^{-1}\left(1 / p_{\varepsilon}\right)(x, D) \exp \left[\varepsilon\langle D\rangle_{h}^{1 / \kappa}\right]$, then $\tilde{Q}_{\varepsilon}$ maps continuously $L_{\kappa, \varepsilon}^{2}$ to $H_{\kappa, \varepsilon}^{m^{\prime}}$ and satisfies $\tilde{Q}_{\varepsilon} p(x, D)=I$ on $H_{k, \varepsilon}^{m}$ if $|\varepsilon| \leq \varepsilon_{0}$. Here we have assumed that $N_{0} \geq \hat{N}_{m^{\prime}}+r+l_{3}+2[n / 2]+3$, where $l_{3}=$ $\left[\left(m-m^{\prime}+1\right) / \rho\right]$. It is easy to see that $Q_{\varepsilon}=Q_{\varepsilon^{\prime}}$ on $H_{\kappa, \varepsilon}^{m-m^{\prime}}$ if $\varepsilon \geq \varepsilon^{\prime}$ and $\tilde{Q}_{\varepsilon}=Q_{\varepsilon}$ on $H_{\kappa, \varepsilon}^{m-m^{\prime}}$, which proves the first part of the proposition. Choose a symbol $\Lambda(x, \xi)$ satisfying

$$
\begin{aligned}
& \left|\Lambda_{(\beta)}^{(\alpha)}(x, \xi)\right| \leq C_{d_{2}} A_{d_{2}}^{|\alpha|+|\beta|}(|\alpha|+|\beta|) !\langle\xi\rangle_{h}^{1 / \kappa-|\alpha|}, \\
& \inf _{L>0} \sup _{|\xi| \geq L}|\Lambda(x, \xi)|\langle\xi\rangle_{h}^{-1 / \kappa}<2, \\
& \inf _{L>0} \sup _{(x, \xi) \in \mathscr{C}_{1},|\xi| \geq L} \Lambda(x, \xi)\langle\xi\rangle_{h}^{-1 / \kappa}<-1, \\
& \sup _{L>0} \inf _{(x, \xi) \in \mathscr{C}_{2},|\xi| \geq L} \Lambda(x, \xi)\langle\xi\rangle_{h}^{-1 / \kappa}>1 .
\end{aligned}
$$

For example, let $\varphi(x, \xi)$ be a function in $C^{1}\left(T^{*} \mathbb{R}^{n}\right)$ such that the first order derivatives of $\varphi$ are bounded, and $|\varphi(x, \xi)|<5 / 3, \varphi(x, \xi)$ $=-4 / 3$ in $\mathscr{C}_{1} \cap\{|\xi| \geq 1 / 2\}$ and $\varphi(x, \xi)=4 / 3$ in $\mathscr{C}_{2} \cap\{|\xi| \geq 1 / 2\}$. Put 
$\varphi,(x, \xi)=E_{j} * \varphi(x, \xi)$ and $\Lambda(x, \xi)=\varphi_{j}\left(x, \xi /\langle\xi\rangle_{h}\right)\langle\xi\rangle_{h}^{1 / \kappa}$, where $E_{j}(x, \xi)=$ $j^{n}(4 \pi)^{-n} \exp \left[-j\left(|x|^{2}+|\xi|^{2}\right) / 4\right]$. If $j$ is sufficiently large, then $\Lambda(x, \xi)$ satisfies $(3.8)-(3.11)$. Let symbols $p_{\Lambda}^{a}(x, \xi)$ and $r_{\Lambda}^{a}(x, \xi)$ be as defined in Lemma 3.1 for $0 \leq a \leq \hat{a}_{d_{2}} A^{-1 / \kappa}$, where $\hat{a}_{d_{2}}$ is a positive constant. By Lemma $3.1 r_{\Lambda}^{a}(x, D)$ maps continuously $L_{\kappa, \varepsilon}^{2}$ to $L_{\kappa, 2 \varepsilon_{1}}^{2}$ for $|\varepsilon| \leq \varepsilon_{1} \equiv \hat{\varepsilon}_{d_{2}} A^{-1 / \kappa}$, where $\hat{\varepsilon}_{d_{2}}>0$. Applying the same argument as in the first part of the proof to $p_{\Lambda}^{a}$ instead of $p$, we can show that there is an operator $Q_{\Lambda}^{a}$ which maps continuously $L_{\kappa, \varepsilon}^{2}$ to $H_{\kappa, \varepsilon}^{m^{\prime}}$ and satisfies $Q_{\Lambda}^{a} p_{\Lambda}^{a}=I$ on $H_{\kappa, \varepsilon}^{m}$, if $|\varepsilon|<\varepsilon_{0} / 4$ and $h \geq h_{p, d_{2}}\left(a, 1 / d_{0}, d_{1}, B\right)$, and if $|\varepsilon| B \leq c_{d_{1}}^{\prime}$ and $a C_{d_{2}} A_{d_{2}} B \leq c_{d_{1}}^{\prime}$ when $\delta=1-1 / \kappa$ or $\rho=1 / \kappa$, and if $B \leq B^{\prime}$ $\left(d_{1}\right)$ when $\rho=\delta$, where $h_{p, d_{2}}\left(a, 1 / d_{0}, d_{1}, B\right), c_{d_{1}}^{\prime}$ and $B^{\prime}\left(d_{1}\right)$ are positive constants. Here we have assumed that $N_{0} \geq \hat{N}_{m-m^{\prime}}+2 r+l_{1}+l_{2}, N_{0} \geq \hat{N}_{m}$ $+2 r$ and $N_{0} \geq \hat{N}_{m^{\prime}}+2 r+l_{3}+2[n / 2]+3$. Lemma 2.14 and the same argument as in the first part of the proof show that ${ }^{R}\left(e^{-a \Lambda}\right)(x, D)$ $\left(e^{a \Lambda}\right)(x, D)$ can be written as ${ }^{R}\left(e^{-a \Lambda}\right)(x, D)\left(e^{a \Lambda}\right)(x, D)=1+\hat{q}_{\Lambda}^{a}(x, D)$ $+\hat{r}_{\Lambda}^{a}(x, D)$ and that $\left(1+\hat{q}_{\Lambda}^{a}(x, D)+\hat{r}_{\Lambda}^{a}(x, D)\right)$ has the inverse on $L_{\kappa, \varepsilon}^{2}$ if $|\varepsilon| \leq \varepsilon_{0}$ and $h \geq h_{d_{2}}\left(a, \varepsilon_{0}\right)$. In fact, using an oscillatory integral, we can estimate a symbol $\sigma\left(\exp \left[\varepsilon\langle D\rangle_{h}^{1 / \kappa}\right] \hat{r}_{\Lambda}^{a}(x, D) \exp \left[-\varepsilon\langle D\rangle_{h}^{1 / \kappa}\right]\right)(x, \xi)$. Let $0 \leq a \leq \min \left(\hat{a}_{d_{2}} A^{-1 / \kappa}, \varepsilon_{0} / 12, \varepsilon_{1} / 3\right)$, and put $u=Q \chi_{1}(x, D) f\left(\in H_{\kappa,-a}^{m}\right)$ for $f \in H_{\kappa,-a}^{m-m^{\prime}}$. By Lemma 2.10, Proposition 2.12 and (3.10), we have $\left(e^{a \Lambda}\right)(x, D) \chi_{1}(x, D) f \in H^{m-m^{\prime}}$. Similarly, Propositions 2.8 and 2.12 and (3.11) imply that $\chi_{2}(x, D)^{R}\left(e^{-a \Lambda}\right)(x, D)$ maps continuously $H_{\kappa, \varepsilon}^{s}$ to $H_{\kappa, \varepsilon+a}^{s}$ if $|\varepsilon| \leq \varepsilon_{0}$. Thus we have

$$
\begin{aligned}
& \chi_{2} Q \chi_{1} f=\chi_{2}{ }^{R}\left(e^{-a \Lambda}\right) Q_{\Lambda}^{a} p_{\Lambda}^{a} e^{a \Lambda}\left(1+\hat{q}_{\Lambda}^{a}+\hat{r}_{\Lambda}^{a}\right)^{-1} u \\
& =\chi_{2}{ }^{R}\left(e^{-a \Lambda}\right) Q_{\Lambda}^{a}\left(e^{a \Lambda} p{ }^{R}\left(e^{-a \Lambda}\right)-r_{\Lambda}^{a}\right) e^{a \Lambda}\left(1+\hat{q}_{\Lambda}^{a}+\hat{r}_{\Lambda}^{a}\right)^{-1} u \\
& =\chi_{2}{ }^{R}\left(e^{-a \Lambda}\right) Q_{\Lambda}^{a} e^{a \Lambda} \chi_{1} f-\chi_{2}{ }^{R}\left(e^{-a \Lambda}\right) Q_{\Lambda}^{a} r_{\Lambda}^{a} e^{a \Lambda}\left(1+\hat{q}_{\Lambda}^{a}+\hat{r}_{\Lambda}^{a}\right)^{-1} u \in H_{\kappa, a}^{m^{\prime}},
\end{aligned}
$$

which completes the proof.

Q.E. D.

Let $\left(x^{0}, \xi^{0}\right) \in T^{*} \boldsymbol{R}^{n} \backslash 0$ and $\left|\xi^{0}\right|=1$, and let $\mathscr{C}$ be a convex conic neighborhood of $\left(x^{0}, \xi^{0}\right)$. Choose a neighborhood $U$ of $x^{0}$ and a conic neighborhood $\Gamma$ of $\xi^{0}$ so that $U \times \Gamma \subset \mathscr{C}$. Moreover, let $\mathscr{C}_{1}$ be a conic neighborhood of $\left(x^{0}, \xi^{0}\right)$ such that $\mathscr{C}_{1} \Subset U \times \Gamma$. Choose $\phi_{1}$ $(x) \in \mathscr{D}^{\left(\kappa^{\prime}\right)}$ and $\psi_{2}(\xi) \in \mathscr{E}^{\left(\kappa^{\prime}\right)}$ for a fixed $\kappa^{\prime}<\kappa$ so that $\phi_{2}(\xi)$ is positively homogeneous of degree 0 for $|\xi| \geq 1,0 \leq \phi_{1}(x) \phi_{2}(\xi) \leq 1$, supp $\phi_{1}(x)$ $\phi_{2}(\xi) \cap\{|\xi|=1\} \Subset U \times \Gamma$ and $\phi_{1}(x) \phi_{2}(\xi)=1$ on $\mathscr{C}_{1} \cap\{|\xi| \geq 1\}$. Let 
$\sigma(\xi) \in \mathscr{E}^{\left(x^{\prime}\right)}$ be a function such that $\sigma(\xi)=0$ for $|\xi| \leq 1$ and $\sigma(\xi)=1$ for $|\xi| \geq 2$, and write $\sigma_{h}(\xi)=\sigma(\xi / h)$ for $h \geq 1$. We set

$$
\begin{aligned}
& X(x)=\left(1-\phi_{1}(x)\right) x^{0}+\phi_{1}(x) x, \\
& \Xi_{h}(\xi)=\left\{h\left(1-\sigma_{h}(\xi)\right)+\sigma_{h}(\xi)\left(1-\phi_{2}(\xi)\right)|\xi|\right\} \xi^{0}+\sigma_{h}(\xi) \psi_{2}(\xi) \xi, \\
& \tilde{p}_{h}(x, \xi)=p\left(X(x), \Xi_{h}(\xi)\right) .
\end{aligned}
$$

Then it is obvious that $\tilde{p}_{h}(x, \xi)=p(x, \xi)$ if $(x, \xi) \in \mathscr{C}_{1}$ and $|\xi| \geq 2 h$.

Lemma 3.5. Assume that $h \geq 1$ and that

$$
\left|\lambda \xi^{0}+\xi\right| \geq(\lambda+|\xi|) / 2 \text { for } \lambda>0 \text { and }(x, \xi) \in \mathscr{C} \text {. }
$$

Then we have $\left(X(x), \Xi_{h}(\xi)\right) \in \mathscr{C},\left|\Xi_{h}(\xi)\right| \geq h / 2$ and

$$
(2 \sqrt{5})^{-1}\langle\xi\rangle_{h} \leq\left|\Xi_{h}(\xi)\right| \leq\left\langle\Xi_{h}(\xi)\right\rangle \leq \sqrt{2}\langle\xi\rangle_{h}
$$

Lemma 3.6. Assume that (3.12) is satisfied. If a symbol $p(x, \xi)$ satisfies

$$
\begin{array}{r}
\left|p_{(\beta)}^{(\alpha)}(x, \xi)\right| \leq C A^{|\alpha|+|\beta|}(|\alpha|+|\beta|) !^{\kappa}\langle\xi\rangle^{m-|\alpha|} \quad \text { for }(x, \xi) \in \mathscr{C} \\
\text { with }|\xi| \geq h_{0}(\geq 1),
\end{array}
$$

then

$$
\left|\tilde{p}_{h(\beta)}^{(\alpha)}(x, \xi)\right| \leq C\left(C, 1 / A_{1}\right) A_{1}^{|\alpha|+|\beta|}(|\alpha|+|\beta|) !^{\mid \kappa}\langle\xi\rangle_{h}^{m-|\alpha|}
$$

for $h \geq 2 h_{0}$ and $(x, \xi) \in T^{*} \boldsymbol{R}^{n}$, where $A_{1} \equiv C_{\sigma, \psi_{1}, \psi_{2}} A$ and $C_{0, \psi_{1}, \psi_{2}}>0$.

Proof. It is sufficient to verify that

$$
\begin{array}{r}
\left|\partial_{\xi}^{\alpha} D_{x}^{\beta} p_{(\gamma)}^{(\gamma)}\left(X(x), \Xi_{h}(\xi)\right)\right| \leq(2 \sqrt{5})^{|m|} C A_{1}^{|\alpha|+|\beta|}(2 \sqrt{5} A)^{|\gamma|+|\nu|} \\
\quad \times(|\alpha|+|\beta|+|\gamma|+|\nu|) !^{\kappa}\langle\xi\rangle_{h}^{m-|\alpha|-|\gamma|} \sum_{k=0}^{|\alpha|+|\beta|} b^{k} / k !^{\kappa-1}
\end{array}
$$

for $h \geq 2 h_{0}$, where $b \equiv b\left(1 / A_{1}\right)$. (3.13) can be proved by induction on $|\alpha|+|\beta|$ if $A_{1} \geq C_{\sigma, \psi_{1}, \psi_{2}} A$.

Q. E. D.

Lemma 3.7. Assume that (3.12) is satisfied. If a symbol $p(x, \xi)$ satisfies

$$
|p(x, \xi)| \geq d_{0}\langle\xi\rangle^{m^{\prime}},
$$

$$
\left|p_{(\beta)}^{(\alpha)}(x, \xi) / p(x, \xi)\right| \leq d_{1} B^{|\alpha|+|\beta|}\langle\xi\rangle^{\delta|\beta|-\rho|\alpha|}
$$

for $(x, \xi) \in \mathscr{C}$ with $|\xi| \geq h_{0}(\geq 1)$ and $|\alpha|,|\beta| \leq N_{0}$, then

$$
\left|\tilde{p}_{h}(x, \xi)\right| \geq(2 \sqrt{5})^{-\left|m^{\prime}\right|} d_{0}\langle\xi\rangle_{h}^{m^{\prime}}
$$




$$
\text { for }(x, \xi) \in T^{*} \boldsymbol{R}^{n} \text { and } h \geq 2 h_{0},
$$

$$
\begin{aligned}
& \left|\tilde{p}_{h(\beta)}^{(\alpha)}(x, \xi) / \tilde{p}_{h}(x, \xi)\right| \leq d_{1} B_{1}^{|\alpha|+|\beta|}\langle\xi\rangle_{h}^{\delta|\beta|-\rho \mid \alpha_{1}} \\
& \text { for }(x, \xi) \in T^{*} \boldsymbol{R}^{n},|\alpha|,|\beta| \leq N_{0} \text { and } h \geq h\left(1 / B_{1}, h_{0}, N_{0}\right),
\end{aligned}
$$

where $B_{1}=C_{\sigma, \psi_{1}, \psi_{2}} B, C_{\sigma, \psi_{1}, \psi_{2}}>0$ and $h\left(1 / B_{1}, h_{0,} N_{0}\right)>0$.

Proof. (3.16) is obvious. We can prove by induction on $|\alpha|+|\beta|$ that

$$
\begin{aligned}
& \left|\partial_{\xi}^{\alpha} D_{x}^{\beta} p_{(\nu)}^{(r)}\left(X(x), \Xi_{h}(\xi)\right) / \tilde{p}_{h}(x, \xi)\right| \\
& \leq d_{1} B_{1}^{|\alpha|+|\beta|}(2 \sqrt{5} B)^{|r|+|\nu|}\langle\xi\rangle_{h}^{\delta(|\beta|+|\nu|)-\rho(|\alpha|+|\gamma|)}
\end{aligned}
$$

if $h \geq h\left(1 / B_{1}, h_{0}, N_{0}\right),|\alpha|+|\gamma| \leq N_{0}$ and $|\beta|+|\nu| \leq N_{0}$, using $\rho<1$ and $\delta>0$. This proves (3.17).

Q. $\mathbb{E}$ 。

Proposition 3. 8. Let $\left(x^{0}, \xi^{0}\right) \in T^{*} \mathbb{R}^{n} \backslash 0$, and let $\mathscr{C}$ be a conic neighborhood of $\left(x^{0}, \xi^{0}\right)$. Assume that $0<\delta \leq 1-1 / \kappa, 1 / \kappa \leq \rho<1$ and $\delta<\rho$, and that $p(x, \xi) \in S_{*}^{m}$ satisfies (3.14) and (3.15) for $(x, \xi) \in \mathscr{C}$ with $|\xi| \geq h_{0}(\geq 1)$ and $|\alpha|,|\beta| \leq N_{0}$, and $p(x, D)$ is properly supported, where $N_{0}$ is a sufficiently large positive integer, $m, m^{\prime} \in \mathbb{R}$ and $h_{0}, d_{0}, d_{1}$ and $B$ are positive constants. Moreover assume that $\delta<1-1 / \kappa$ and $\rho>1 / \kappa$ if $*=(\kappa)$. Then there is an operator $Q$, which maps continuously $\mathscr{D}^{* \prime}$ to $\mathscr{D}^{* \prime}$, such that

$$
\begin{aligned}
& \left(x^{0}, \xi^{0}\right) \notin W F_{*}(p Q f-f) \cup W F_{*}(Q p f-f) \quad \text { for } f \in \mathscr{D}^{*^{\prime}}, \\
& \left(x^{0}, \xi^{0}\right) \notin W F_{*}(Q f) \quad \text { if }\left(x^{0}, \xi^{0}\right) \notin W F_{*}(f) \text { and } f \in \mathscr{D}^{*^{\prime}} .
\end{aligned}
$$

Remark. (i) Taniguchi [25] essentially proved the proposition by his method of multi-products of pseudo-differential operators, and constructed $Q$ as a pseudo-differential operator. (ii) The proposition implies that $p(x, D)$ has a microlocal parametrix at $\left(x^{0}, \xi^{0}\right)$ modulo $\mathscr{E}^{*}$ and, therefore, $p(x, D)$ is hypoelliptic at $\left(x^{0}, \xi^{0}\right)$ (in $\mathscr{\mathscr { C }}$ ) with respect to $\mathscr{E}^{*}$. (iii) When $\rho=1$ or $\delta=0$, the proposition is valid, modifying $\rho$ and $\delta$.

Proof. We may assume that for any $A>0$ there is $C \equiv G_{A}>0$ (resp. there are $A>0$ and $C>0$ ) such that

$$
\left|p_{(\beta)}^{(\alpha)}(x, \xi)\right| \leq C A^{|\alpha|+|\beta|}(|\alpha|+|\beta|) !^{\kappa}\langle\xi\rangle^{m-|\alpha|} \text { for }(x, \xi) \in T^{*} \mathbb{R}^{n}
$$

if $*=(\kappa)$ (resp. if $*=\{\kappa\})$, and that $\left|\xi^{0}\right|=1$ and (3.12) is satisfied. 
By Lemmas 3.5-3.7, Proposition 3.4 can be applicable and there is an operator $\tilde{Q}_{h}$, which maps continuously $L_{\kappa, \varepsilon}^{2}$ to $H_{\kappa \varepsilon \varepsilon}^{m^{\prime}}$ and $H_{\kappa, \varepsilon}^{m-m^{\prime}}$ to $H_{\kappa, \varepsilon}^{m}$ and satisfies $\tilde{Q}_{h} \tilde{p}_{h}=I$ on $H_{\kappa, \varepsilon}^{m}$ and $\tilde{p}_{h} \tilde{Q}_{h}=I$ on $H_{\kappa, \varepsilon}^{m-m^{\prime}}$ if $\varepsilon$ and $h$ satisfy the following conditions;

$$
\begin{aligned}
& |\varepsilon|<\varepsilon_{1} \equiv \hat{\varepsilon}_{U \times \Gamma, \mathscr{C}_{1}} A^{-1 / \kappa} \text { and } h \geq h_{p, B, U \times \Gamma, \mathscr{C}_{1}}\left(1 / d_{0}, d_{1}, h_{0}\right) \text {, and } \\
& |\varepsilon| B \leq c_{d_{1}, U \times \Gamma, \mathscr{C}_{1}} \text { when } \delta=1-1 / \kappa \text { or } \rho=1 / \kappa .
\end{aligned}
$$

Fix $h>0$ so that $h$ satisfies (3.18). If $*=(\kappa)$, then $A$ can tend to zero. So, for any $\varepsilon \in \boldsymbol{R}$ we can define

$$
\tilde{Q} f=\tilde{Q}_{h^{\prime}} f-\tilde{Q}_{h}\left(\tilde{p}_{h}-\tilde{p}_{h^{\prime}}\right) \tilde{Q}_{h^{\prime}} f \quad \text { for } f \in H_{\kappa, \varepsilon}^{m-m^{\prime}},
$$

where $h^{\prime}(\geq h)$ is sufficiently large according to $|\varepsilon|$, when $*=(\kappa)$. In fact, $\tilde{p}_{h} \tilde{Q} f=f$ and $\tilde{Q} \tilde{p}_{h} f=f+\left(\tilde{Q}_{h^{\prime}}-\tilde{Q}_{h}\right)\left(\tilde{p}_{h}-\tilde{p}_{h^{\prime}}\right) f-\tilde{Q}_{h}\left(\tilde{p}_{h}-\tilde{p}_{h^{\prime}}\right) \tilde{Q}_{h^{\prime}}$ $\left(\tilde{p}_{h}-\tilde{p}_{h^{\prime}}\right) f=f$ for $f \in H_{k, \varepsilon}^{m-m^{\prime}}$, since $\tilde{p}_{h}(x, \xi)=\tilde{p}_{h^{\prime}}(x, \xi)$ for $|\xi| \geq 2 h^{\prime}$. This implies that $\tilde{Q}$ does not depend on $h^{\prime}$. When $*=\{\kappa\}$, we define $\tilde{Q}=\tilde{Q}_{h}$. Then we have

$$
\tilde{Q} f-\tilde{Q}_{h^{\prime}} f=\tilde{Q} f-\tilde{Q}_{h^{\prime}} \tilde{p}_{h} \tilde{Q} f=\tilde{Q}_{h^{\prime}}\left(\tilde{p}_{h^{\prime}}-\tilde{p}_{h}\right) \tilde{Q} f \in H_{\kappa, \varepsilon_{2}}^{m^{\prime}}
$$

if $f \in H_{\kappa, \varepsilon}^{m-m^{\prime}}, 0<\varepsilon_{2}<\varepsilon_{1}$ and $|\varepsilon|<\varepsilon_{1}$, and if $h^{\prime}(\geq h)$ is sufficiently large according to $\varepsilon_{1}$ (or $A^{-1}$ ) when $*=(\kappa)$, and if $\varepsilon$ and $h^{\prime}(\geq h)$ satisfy (3.18) when $*=\{\kappa\}$, modifying $\hat{\varepsilon}_{U \times \Gamma \cdot \mathscr{C}_{1}}$. Let $\varphi(x) \in \mathscr{D}^{(\kappa)}$ and $\chi(x, \xi)$ $\in \mathscr{E}^{(\kappa)}\left(T^{*} \boldsymbol{R}^{n}\right)$ be functions such that $\varphi(x)=1$ in a neighborhood of $U, \chi(x, \xi)$ is positively homogeneous of degree 0 in $\xi$ for $|\xi| \geq 1$, $0 \leq \chi(x, \xi) \leq 1, \operatorname{supp} \chi(x, \xi) \cap\{|\xi|=1\} \Subset \mathscr{C}_{1}$ and $\chi(x, \xi)=1$ if $(x, \xi)$ belongs to a conic neighborhood of $\left(x^{0}, \xi^{0}\right)$ and $|\xi| \geq 1$. We write $\chi \Subset \mathscr{C}_{1}$ for $\left(x^{0}, \xi^{0}\right)$ if $\chi(x, \xi)$ has the above properties. Let $\chi_{1}(x, \xi)$ $\in \mathscr{E}^{(x)}\left(T^{*} \boldsymbol{R}^{n}\right)$ satisfy $\chi_{1} \Subset \chi$ for $\left(x^{0}, \xi^{0}\right)$, i. e., $\chi_{1} \Subset\{(x, \lambda \xi) ; \chi(x, \xi)=1$, $|\xi| \geq 1$ and $\lambda>0\}$ for $\left(x^{0}, \xi^{0}\right)$. We set $Q f=\tilde{Q} \chi(x, D) \varphi(x) f$. Then it follows from (3.19) that $Q$ maps continuously $\mathscr{D}^{*^{\prime}}$ to $\mathscr{D}^{*^{\prime}}$ and $\mathscr{E} *$ to $\mathscr{E}^{*}$. In fact, if $*=(\kappa)$, then $A$ can tend to zero and $\varepsilon_{1}$ can tend to $+\infty$. So, for any $\varepsilon_{1}>0 \quad \tilde{Q}_{h^{\prime}}$ maps continuously $L_{\kappa, \varepsilon}^{2}$ to $H_{\kappa, \varepsilon}^{m^{\prime}}$ if $*=(\kappa),|\varepsilon|<\varepsilon_{1}$ and $h^{\prime}$ is sufficiently large. We have also

$$
\chi_{1} \varphi p Q f-\chi_{1} \varphi f=\chi_{1} \varphi(\chi \varphi-1) f+\chi_{1} \varphi\left(p-\tilde{p}_{h}\right) Q f \in \mathscr{E} *
$$

for $f \in \mathscr{D}^{*^{\prime}}$. Taking $h^{\prime}$ sufficiently large according to $\chi_{1}$ and $\chi_{9}$ it follows from Proposition 3.4 (pseudo-locality of $\tilde{Q}_{h^{\prime}}$ ) and (3.19) that

$$
\chi_{1} Q p f-\chi_{1} f=\chi_{1} \tilde{Q}_{h^{\prime}}\left(\chi \varphi p-\tilde{p}_{h}\right) f+\chi_{1}\left(\tilde{Q}-\tilde{Q}_{h^{\prime}}\right)\left(\chi \varphi p-\tilde{p}_{h}\right) f \in L_{\kappa, \varepsilon_{1}}^{2}
$$


if $f \in L_{\kappa, \varepsilon}^{2}$ and $|\varepsilon|<\varepsilon_{1}$, and if $|\varepsilon|$ is sufficiently small according to $B$, $d_{1}, \chi_{1}$ and $\chi$ when $\delta=1-1 / \kappa$ or $\rho=1 / \kappa$, modifying $\hat{\varepsilon}_{U \times \Gamma \cdot \mathscr{G}_{1}}$. This implies that $\chi_{1} Q p f-\chi_{1} f \in \mathscr{E}^{*}$ for $f \in \mathscr{D}^{*}$, since $\delta<1-1 / \kappa$ and $\rho>1 / \kappa$ when $*=(\kappa)$. Assume that $f \in \mathscr{D}^{*^{\prime}}$ and $\left(x^{0}, \xi^{0}\right) \notin W F_{*}(f)$. We may assume that $f \in \mathscr{E}^{*}$. Then there is $\chi_{2}(x, \xi) \in \mathscr{E}^{(x)}\left(T^{*} \boldsymbol{R}^{n}\right)$ such that $\chi_{2} \Subset \chi_{1}$ for $\left(x^{0}, \xi^{0}\right)$ and $\chi_{2}(x, D) f \in \mathscr{E}^{*}$. In fact, by definition there is $\tilde{\chi}(x, \xi) \in \mathscr{E}^{(x)}\left(T^{*} \boldsymbol{R}^{n}\right)$ such that $\tilde{\chi} \Subset \chi_{1}$ for $\left(x^{0}, \xi^{0}\right)$ and ${ }^{R} \tilde{\chi}(x, D) f \in$ $\mathscr{E}^{*}$. If $\chi_{2} \Subset \tilde{\chi}$ for $\left(x^{0}, \xi^{0}\right)$, Lemma 2.15 implies that $\chi_{2}(x, D){ }^{R} \tilde{\chi}$ $(x, D) f-\chi_{2}(x, D) f \in \mathscr{E}^{*}$. Let $\chi_{3}(x, \xi) \in \mathscr{E}^{(x)}\left(T^{*} \boldsymbol{R}^{n}\right)$ satisfy $\chi_{3} \Subset \chi_{2}$ for $\left(x^{0}, \xi^{0}\right)$. Taking $h^{\prime}$ sufficiently large according to $\chi_{2}$ and $\chi_{3}$, Lemma 2. 15 and Proposition 3. 4 give

$$
\chi_{3}(x, D) \tilde{Q}_{h^{\prime}} \chi(x, D) \varphi(x)\left(1-\chi_{2}(x, D)\right) f \in L_{\kappa, \varepsilon_{1}\left(h^{\prime}\right)}^{2},
$$

where $\varepsilon_{1}\left(h^{\prime}\right)>0$ and $\varepsilon_{1}\left(h^{\prime}\right) \rightarrow \infty$ as $h^{\prime} \rightarrow \infty$ when $*=(\kappa)$. In fact, we can write

$$
\begin{aligned}
& \chi \varphi\left(1-\chi_{2}\right) f=b_{1} \varphi\left(1-\chi_{2}\right) f+b_{2} \varphi\left(1-\chi_{2}\right) f, \\
& b_{1}(x, \xi)=\chi(x, \xi)\left(1-\chi_{4}(x, \xi)\right), b_{2}(x, \xi)=\chi(x, \xi) \chi_{4}(x, \xi),
\end{aligned}
$$

where $\chi_{4}(x, \xi) \in \mathscr{E}^{(x)}\left(T^{*} \boldsymbol{R}^{n}\right)$ satisfies $\chi_{3} \Subset \chi_{4} \Subset \chi_{2}$ for $\left(x^{0}, \xi^{0}\right)$. On the other hand, we have $\chi_{3}(x, D) Q \chi_{2}(x, D) f \in \mathscr{E}^{*}$. Therefore, by (3. 19) we have $\chi_{3}(x, D) Q f \in \mathscr{E}^{*}$. Corollary 2 of Lemma 2.15 implies that $\left(x^{0}, \xi^{0}\right) \notin W F_{*}(Q f)$.

Q.E. D.

\section{§4. The Microlocal Cauchy Problem}

Modifying $p(x, D)$ and using pseudo-differential operators of infinite order, we shall reduce the problem in Gevrey classes to the problem in the Sobolev spaces and construct the inverses of the reduced operators in this section. Then we can construct microlocal parametrices of the microlocal Cauchy problem in Gevrey classes and prove microlocal well-posedness (see Theorem 4.11 below). Theorem 1.4 easily follows from Theorem 4.11 (see §5). In this section we assume that $p(x, \xi)$ satisfies the conditions $(\mathrm{A}-1)$ and $(\mathrm{A}-2)$ with $\kappa_{1}$ replaced by $\kappa(>1)$. Let $z^{0}=\left(x^{0}, \xi^{0}\right) \in T^{*} \boldsymbol{R}^{n} \backslash 0, \quad\left|\xi^{0}\right|=1$ and $\vartheta \in$ $T_{z^{0}}\left(T^{*} R^{n}\right)$, and assume that $p_{m}(x, \xi)$ is microhyperbolic with respect to $\vartheta$ at $z^{0}$.

Lemma 4.1. ([30], [33]). Let $M \Subset \Gamma\left(p_{m z}{ }^{0}, \vartheta\right)$. Then there is a 
neighborhood $\mathscr{U}$ of $z^{0}$ in $T^{*} \boldsymbol{R}^{n} \backslash 0$ such that $p_{m}$ is microhyperbolic with respect to $\tilde{\vartheta} \in M$ at $z \in \mathscr{U}$, and $M \subset \Gamma\left(p_{m z}, \vartheta\right)$ for $z \in \mathscr{U}$.

Define for $v \in T_{z^{0}}\left(T^{*} R^{n}\right)$

$$
p_{m}(x, \xi ; v, t)=\sum_{j=0}^{l}(-i t v)^{j} p_{m}(x, \xi) / j !,
$$

where $l=\mu\left(z^{0}\right)$ and $v$ is regarded as a vector field. By definition there are a neighborhood $\mathscr{U}$ of $z^{0}$ in $T^{*} \boldsymbol{R}^{n} \backslash 0$ and positive constants $c$ and $t_{0}$ such that

$$
\left|p_{m}(x, \xi ; \vartheta, t)\right| \geq c t^{l} \quad \text { for }(x, \xi) \in \mathscr{U} \text { and } 0 \leq t \leq t_{0} .
$$

Lemma 4.2. Let $M$ be a compact subset of $\Gamma\left(p_{m z^{0}}, \vartheta\right)$. Modifying $\mathscr{U}, c$ and $t_{0}$, we have

$$
\begin{aligned}
& \left|p_{m}(x, \xi ; v, t)\right| \geq c t^{l} \\
& \left|\partial_{t}^{j} p_{m(\beta)}^{(\alpha)}(x, \xi ; v, t) / p_{m}(x, \xi ; v, t)\right| \leq C(\alpha, \beta) t^{-|\alpha|-|\beta|-j}
\end{aligned}
$$

if $(x, \xi) \in \mathscr{U}, v \in M$ and $0<t \leq t_{0}$.

Remark. Without applying the Malgrange preparation theorem, we can also prove the lemma if only $p_{m}(x, \xi) \in C^{l+\delta}\left(T^{*} R^{n}\right)$ and $0<\delta$ $(<1)$.

Proof. Let $\chi(x, \xi) \in C_{0}^{\infty}\left(T^{*} \boldsymbol{R}^{n}\right)$ satisfy supp $\chi \subset\{|x|+|\xi| \leq 2 h\}$ and $\chi(x, \xi)=1$ for $|x|+|\xi| \leq h$, where $h>0$, and define an almost analytic extension of $p_{m}(x, \xi)$ by

$$
\begin{aligned}
& p_{m}(x+i y, \xi+i \eta)=\sum_{\alpha, \beta}(\alpha ! \beta !)^{-1}(i \eta)^{\alpha}(-y)^{\beta} p_{m(\beta)}^{(\alpha)}(x, \xi) \\
& \quad \times \chi\left(b_{|\alpha|+|\beta|} y, b_{|\alpha|+|\beta|} \eta\right) \text { for }(x, \xi) \in \overline{\mathscr{U}} \text { and }(y, \eta) \in \boldsymbol{R}^{n} \times \boldsymbol{R}^{n},
\end{aligned}
$$

where $b_{0}=1$ and $\left\{b_{k}\right\} \subset \boldsymbol{R}$ is a rapidly increasing sequence. Then we have

$$
\left|\partial_{t}^{j} \partial_{\xi}^{\alpha} D_{x}^{\beta}\left\{p_{m}((x, \xi)-i t v)-p_{m}(x, \xi ; v, t)\right\}\right| \leq c_{0}|t|^{l+1-j}
$$

if $t \in \boldsymbol{R},|t| \leq t_{0},(x, \xi) \in \mathscr{U}, j \leq l$ and $|\alpha|+|\beta| \leq l$. From Lemma 2.6 in [33] it follows that

$$
\left|p_{m}((x, \xi)-i t v)\right| \geq c_{1} t^{l} \quad \text { for }(x, \xi) \in \mathscr{U}, v \in M \text { and } 0 \leq t \leq t_{0},
$$

modifying $\mathscr{U}$ and $t_{0}$ if necessary, which proves (4.2). Applying the Malgrange preparation theorem, there are a neighborhood $\mathscr{U}_{1}$ of $z^{0}$, $\delta>0, e(z, v, t) \in C^{\infty}\left(\overline{\mathscr{U}}_{1} \times M \times[-\delta, \delta]\right)$ and $a_{j}(z, v) \in C^{\infty}\left(\overline{\mathscr{U}}_{1} \times M\right)(1 \leq j$ $\leq l)$ such that $a_{j}\left(z^{0}, v\right)=0$ for $v \in M$ and $1 \leq j \leq l, p_{m}(z+t v)=e(z, v, t)$ 
$\times g(z, v, t)$ and $e(z, v, t) \neq 0$ for $(z, v, t) \in \overline{\mathscr{U}}_{1} \times M \times[-\delta, \delta]$, and $g(z, v, t)$ $\equiv t^{l}+a_{1}(z, v) t^{l-1}+\cdots+a_{l}(z, v) \neq 0$ for $(z, v, t) \in \overline{\mathscr{U}}_{1} \times M \times \boldsymbol{C}$ and Im $t<0$. In fact, by Mather's proof of the Malgrange preparation theorem we can obtain the above assertion without dividing $M$ (see [30]). Applying Theorem 2 in [32] to $\mathrm{g}(z, v, t)$, we have

$$
\left|\partial_{t}^{j} g_{(\beta)}^{(\alpha)}(x, \xi ; v, t) / g(x, \xi ; v, t)\right| \leq C^{\prime}(\alpha, \beta)|\operatorname{Im} t|^{-|\alpha|-|\beta|-j}
$$

if $(x, \xi) \in \mathscr{U}_{1}, v \in M, t \in C$ and $-1 \leq \operatorname{Im} t<0$. On the other hand, (4.4) yields

$$
\left|\partial_{t}^{j}\left\{p_{m}(x, \xi ; v, t)-e(x, \xi, v,-i t) g(x, \xi, v,-i t)\right)_{\left(\begin{array}{c}
\alpha \\
\beta
\end{array}\right)}^{(\alpha)}\right| \leq C_{0}^{\prime}|t|^{l+1-j}
$$

for $t \in \boldsymbol{R},|t| \leq t_{0},(x, \xi) \in \mathscr{U}, j \leq l$ and $|\alpha|+|\beta| \leq l$, modifying $\mathscr{U}$ if necessary, where $e(x, \xi, v, t+i \tau)$ is an almost analytic extension of $e(x, \xi, v, t)$ in $t$. This proves (4.3) for $j \leq l$ and $|\alpha|+|\beta| \leq l$. It is obvious that (4.3) is valid for $j+|\alpha|+|\beta| \geq l$. $\quad$ Q.E.D.

Corollary. Let $M$ be a compact subset of $\Gamma\left(p_{m z^{0}}, \vartheta\right)$. Then there are a conic neighborhood $\mathscr{C}$ of $z^{0}$ and positive constants $c$ and $t_{0}$ such that

$$
\begin{aligned}
& \left|p_{m}(x, \xi ; v(\xi), t|\xi|)\right| \geq c t^{l}|\xi|^{m}, \\
& \left|p_{m(\beta)}^{(\alpha)}(x, \xi) / p_{m}(x, \xi ; v(\xi), t|\xi|)\right| \leq C(\alpha, \beta) t^{-|\alpha|-|\beta|}|\xi|^{-|\alpha|}
\end{aligned}
$$

if $(x, \xi) \in \mathscr{C}, v \in M$ and $0<t \leq t_{0}$, where $v(\xi)=\left(v_{x} /|\xi|, v_{\xi}\right)$ for $v=\left(v_{x}, v_{\xi}\right)$ $\in \boldsymbol{R}^{n} \times \boldsymbol{R}^{n}$.

Proof. It is easy to see that

$$
p_{m(\beta)}^{(\alpha)}(x, \xi /|\xi|)=\sum_{j=0}^{l}(-t)^{j} \partial_{t}^{j} p_{m(\beta)}^{(\alpha)}(x, \xi /|\xi| ; v, t) / j ! \text {. }
$$

On the other hand,

$$
p_{m}(x, \xi /|\xi| ; v, t)=p_{m}(x, \xi ; v(\xi), t|\xi|)|\xi|^{-m} .
$$

Therefore, we have

$$
\begin{aligned}
& \mid p_{m(\beta)}^{(\alpha)}(x, \xi) / p_{m}(x, \xi ; v(\xi), t|\xi|) \\
& \leq|\xi|^{-|\alpha|} \sum_{j=0}^{l} t^{j}\left|\partial_{t}^{J} p_{m(\beta)}^{(\alpha)}(x, \xi /|\xi| ; v, t) p_{m}(x, \xi /|\xi| ; v, t)\right| / j ! \\
& \leq C^{\prime}(\alpha, \beta) t^{-|\alpha|-|\beta|}|\xi|^{-|\alpha|}
\end{aligned}
$$

if $(x, \xi /|\xi|) \in \mathscr{U}, v \in M$ and $0<t \leq t_{0}$. This completes the proof.

Q.E.D.

Now assume that $1<\kappa \leq \kappa_{0} \equiv \min \left\{2, \mu\left(z^{0}\right) /\left(\mu\left(z^{0}\right)-1\right)\right\}$ if $*=(\kappa)$ and $1<\kappa<\kappa_{0}$ if $*=\{\kappa\}$. Let $\varphi(x, \xi) \in C^{2}\left(T^{*} \boldsymbol{R}^{n} \backslash 0\right)$ be a real-valued 
positively homogeneous function of degree 0 in $\xi$ such that $\varphi\left(z^{0}\right)=0$ and $-H_{\varphi}\left(z^{0}\right) \equiv-\sum_{j=1}^{n}\left\{\left(\partial \varphi / \partial \xi_{\jmath}\right)\left(z^{0}\right)\left(\partial / \partial x_{\jmath}\right)-\left(\partial \varphi / \partial x_{j}\right)\left(z^{0}\right)\left(\partial / \partial \xi_{\jmath}\right)\right\} \in$ $\Gamma\left(p_{m z}, \vartheta\right)$. Choose a compact subset $M$ of $\Gamma\left(p_{m z^{0}}, \vartheta\right)$ so that $\vartheta \in M$ and $-H_{\varphi}\left(z^{0}\right) \in M$. Then there is a neighborhood $\mathscr{U}$ of $z^{0}$ such that $M \Subset \Gamma\left(p_{m z}, \vartheta\right)$ for $z \in \mathscr{U}$. For given $f \in \mathscr{D}^{*^{\prime}}$ with $W F_{*}(f) \cap\{\varphi(x, \xi)$ $<0\} \cap \mathscr{U}=\varnothing$, we shall consider the microlocal Cauchy problem at $z^{0}$ (MCP)

$$
\left\{\begin{array}{l}
p(x, D) u=f, \\
W F_{*}(u) \cap\{\varphi(x, \xi)<0\} \cap \mathscr{U}=\varnothing,
\end{array}\right.
$$

where $u \in \mathscr{D}^{* \prime}$.

Lemma 4.3. Let $M_{1}$ be a compact convex subset of $\Gamma\left(p_{m z^{0}}, \vartheta\right)$ such that $M \Subset M_{1} \Subset \Gamma\left(p_{m z}, \vartheta\right)$ for $z \in \mathscr{U}$. Then there are symbols $\Lambda_{h}(x, \xi)$ $(h \geq 1)$, a convex conic neighborhood $\mathscr{C}$ of $z^{0}$, and positive constants $\varepsilon_{1}, C_{0}$, $A_{0}, c_{1}$ and $c_{2}$ such that

$$
\begin{array}{cl}
\text { (4.5) } & \left|\Lambda_{h}(x, \xi)\right| \leq\langle\xi\rangle_{h}^{1 / \kappa} \quad \text { for } \quad(x, \xi) \in T^{*} \boldsymbol{R}^{n}, \\
(4.6) & \left|\Lambda_{h(\beta)}^{(\alpha)}(x, \xi)\right| \leq C_{0} A_{0}^{|\alpha|+|\beta|}(|\alpha|+|\beta|) !\langle\xi\rangle_{h}^{1 / \kappa-|\alpha|} \\
& \text { for }(x, \xi) \in T^{*} \boldsymbol{R}^{n}, \\
(4.7) & \left|\Lambda_{h}\left(x^{0}, \lambda \xi^{0}\right)\right| \leq c_{1} h^{4} \lambda^{1 / \kappa-4} \quad \text { for } \lambda \geq h, \\
(4.8) & \Lambda_{h}(x, \xi) \leq-\left(\varphi(x, \xi)+\left|x-x^{0}\right|^{2}+|\xi /| \xi\left|-\xi^{0}\right|^{2}\right)\langle\xi\rangle_{h}^{1 / \kappa}+c_{2} h^{1 / \kappa} \\
& \text { for }(x, \xi) \in \mathscr{C} \text { and }|\xi| \geq h, \\
(4.9) \quad & \langle\xi\rangle_{h}^{-1 / \kappa} \sum_{j=1}^{n}\left\{|\xi|\left(\partial \Lambda_{h} / \partial \xi_{j}\right)(x, \xi)\left(\partial / \partial x_{j}\right)\right. \\
& \left.-\left(\partial \Lambda_{h} / \partial x_{j}\right)(x, \xi)\left(\partial / \partial \xi_{j}\right)\right\}+\sum_{j=1}^{n}\left\{y,\left(\partial / \partial x_{j}\right)+\eta_{j}\left(\partial / \partial \xi_{j}\right)\right\} \in M_{1} \\
& \text { for }(x, \xi) \in \mathscr{C},|\xi| \geq h \text { and }|y|^{2}+|\eta|^{2} \leq \varepsilon_{1}^{2} .
\end{array}
$$$$
\text { (4.9) }\langle\xi\rangle_{h}^{-1 / \kappa} \sum_{j=1}^{n}\left\{|\xi|\left(\partial \Lambda_{h} / \partial \xi_{j}\right)(x, \xi)\left(\partial / \partial x_{j}\right)\right.
$$

Proof. Put

$$
\begin{aligned}
\varphi_{1}(x, \xi)= & \left(x-x^{0}\right) \cdot \nabla_{x} \varphi\left(z^{0}\right)+\xi \cdot \nabla_{\xi} \varphi\left(z^{0}\right) \\
& +B_{\varphi}\left(\left|x-x^{0}\right|^{2}+\left|\xi-\xi^{0}\right|^{2}\right), \\
\varphi_{2}(x, \xi)= & \varphi_{1}(x, \xi)\left(1+\varphi_{1}(x, \xi)^{2}\right)^{-1 / 2},
\end{aligned}
$$

and choose $B_{\varphi} \in \boldsymbol{R}$ so that $\varphi(x, \xi) \leq \varphi_{1}(x, \xi)-2\left(\left|x-x^{0}\right|^{2}+\left|\xi-\xi^{0}\right|^{2}\right)$ for $\left|x-x^{0}\right|^{2}+\left|\xi-\xi^{0}\right|^{2} \leq 1$ and $|\xi|=1$. If we set $\Lambda_{h}(x, \xi)=-\varphi_{2}(x, \xi)$ $\left.\langle\xi\rangle_{\theta h}\right)\langle\xi\rangle_{h}^{1 / \kappa}$ and $\theta(0<\theta \leq 1)$ is chosen appropriately, we can show that $\Lambda_{h}(x, \xi)$ satisfies $(4.5)-(4.9)$. It is obvious that $\Lambda_{h}(x, \xi)$ satisfies (4.5). Noting that $\varphi_{1}(x, \eta)$ is a polynomial of $(x, \eta)$, there are $L>0$ and $(1 \gg) c>0$ such that $\operatorname{Re} \varphi_{1}(x, \eta)^{2} \geq 0$ if $(x, \eta) \in C^{n} \times$ 
$C^{n} . \quad\left|\operatorname{Re} x-x^{0}\right| \geq L,|\operatorname{Im} x| \leq c\left|\operatorname{Re} x-x^{0}\right|$ and $|\eta| \leq 3$. Then Cauchy's estimates yield

$$
\left|\varphi_{2(\beta)}^{(\alpha)}(x, \eta)\right| \leq C_{\varphi} A_{\varphi}^{|\alpha|+|\beta|}(|\alpha|+|\beta|) !
$$

for $(x, \eta) \in T^{*} \boldsymbol{R}^{n}$ with $1 / 2 \leq|\eta| \leq 2$. From Lemma 2.2 (4.6) easily follows, where $A_{0}$ depends on $\theta$. (4.7) is trivial. It is easy to see that

$$
\begin{aligned}
& \varphi_{2}\left(x, \xi /\langle\xi\rangle_{\theta h}\right)-\varphi(x, \xi) \\
& \geq \varphi_{1}(x, \xi /|\xi|)-\varphi(x, \xi)-\left|\varphi_{1}(x, \xi /|\xi|)-\varphi_{1}\left(x, \xi /\langle\xi\rangle_{\theta h}\right)\right| \\
& \quad-\left|\varphi_{1}\left(x, \xi /\langle\xi\rangle_{\theta h}\right)-\varphi_{2}\left(x, \xi /\langle\xi\rangle_{\theta h}\right)\right|, \\
& \left|\varphi_{1}(x, \xi /|\xi|)-\varphi_{1}\left(x, \xi /\langle\xi\rangle_{\theta h}\right)\right| \leq h^{2}\left|\nabla_{\xi} \varphi\left(z^{0}\right)\right|\langle\xi\rangle_{\theta h}^{-1}|\xi|^{-1} / 2 \\
& \quad+2 h^{2}\left|B_{\varphi}\right|\langle\xi\rangle_{\theta h}^{-1}|\xi|^{-1} \quad \text { for } \xi \neq 0,
\end{aligned}
$$

and that there are $\delta_{\varphi}>0$ and $\theta_{\varphi}>0$ such that $\theta_{\varphi} \leq 1$ and

$$
\begin{aligned}
& \left|\varphi_{1}\left(x, \xi /\langle\xi\rangle_{\theta h}\right)-\varphi_{2}\left(x, \xi /\langle\xi\rangle_{\theta h}\right)\right| \leq\left|\varphi_{1}\left(x, \xi /\langle\xi\rangle_{\theta h}\right)\right|^{3} / 2 \\
& \leq\left|x-x^{0}\right|^{2}+|\xi /| \xi\left|-\xi^{0}\right|^{2}+c_{2}^{\prime} h^{2}\langle\xi\rangle_{h}^{-2}
\end{aligned}
$$

if $0<\theta \leq \theta_{\varphi},\left|x-x^{0}\right|^{2}+|\xi /| \xi\left|-\xi^{0}\right|^{2} \leq \delta_{\varphi}$ and $|\xi| \geq h$, where $c_{2}^{\prime}>0$. Here we have used the inequality that $\left|\xi /\langle\xi\rangle_{\theta h}-\xi /\right| \xi|| \leq \theta^{2} h^{2}\langle\xi\rangle_{\theta h}^{-1}|\xi|^{-1} / 2$. This proves (4.8), taking $\theta \leq \theta_{\varphi}$. A simple calculation gives

$$
\begin{aligned}
\varphi_{2(\beta)}^{(\alpha)}(x, \xi)= & \varphi_{1(\beta)}^{(\alpha)}(x, \xi)\left(1+\varphi_{1}(x, \xi)^{2}\right)^{-3 / 2} \quad \text { for }|\alpha|+|\beta|=1, \\
\varphi_{1(\beta)}^{(\alpha)}(x, \xi)= & \varphi_{(\beta)}^{(\alpha)}\left(z^{0}\right)+2 B_{\varphi}\left(|\beta|\left(x-x^{0}\right)^{\beta} / i+|\alpha|\left(\xi-\xi^{0}\right)^{\alpha}\right) \\
& \text { for }|\alpha|+|\beta|=1, \\
\Lambda_{h}^{\left(e_{j}\right)}(x, \xi)= & -\sum_{k=1}^{n} \varphi_{2}^{\left(e_{k}\right)}\left(x, \xi /\langle\xi\rangle_{\theta h}\right)\left(\delta_{j k}\langle\xi\rangle_{\theta h}^{-1}-\xi_{j} \xi_{k}\langle\xi\rangle_{\theta h}^{-3}\right)\langle\xi\rangle_{h}^{1 / \kappa} \\
& -\varphi_{2}\left(x, \xi /\langle\xi\rangle_{\theta h}\right) \xi_{j}\langle\xi\rangle_{h}^{1 / \kappa-2} / \kappa, \\
\Lambda_{h\left(e_{j}\right)}(x, \xi)= & -\varphi_{2\left(e_{j}\right)}\left(x, \xi /\langle\xi\rangle_{\theta h}\right)\langle\xi\rangle_{h}^{1 / \kappa},
\end{aligned}
$$

where $e_{\jmath}=(0, \cdots, 1, \cdots, 0) \in(\boldsymbol{N} \cup\{0\})^{n}$. Moreover, with $C_{\varphi}>0$, we have

$$
\begin{aligned}
&\left|\varphi_{1}\left(x, \xi /\langle\xi\rangle_{\theta h}\right)\right| \leq C_{\varphi}\left(\left|x-x^{0}\right|^{2}+\left|\xi /\langle\xi\rangle_{\theta h}-\xi^{0}\right|^{2}\right)^{1 / 2} \\
& \text { for }\left|x-x^{0}\right|^{2}+\left|\xi /\langle\xi\rangle_{\theta h}-\xi^{0}\right|^{2} \leq 1, \\
&\left|\sum_{k=1}^{n} \varphi_{2}^{\left(e_{k}\right)}\left(x, \xi /\langle\xi\rangle_{\theta h}\right) \xi_{k}\langle\xi\rangle_{\theta h}^{-1}\right| \leq\left(\left|\nabla_{\xi} \varphi\left(z^{0}\right)\right|+2\left|B_{\varphi}\right|\right) \\
& \times\left|\xi /\langle\xi\rangle_{\theta h}-\xi^{0}\right|,
\end{aligned}
$$

since $\xi^{0} \cdot \nabla_{\xi} \varphi\left(z^{0}\right)=0$. Therefore, we have

$$
\begin{gathered}
\left|\langle\xi\rangle_{h}^{-1 / \kappa}\right| \xi\left|\Lambda_{h}^{(e,)}(x, \xi)+\varphi^{\left(e_{j}\right)}\left(z^{0}\right)\right|+\left|\langle\xi\rangle_{h}^{-1 / \kappa} \Lambda_{h\left(e_{j}\right)}(x, \xi)+\varphi_{\left(e_{j}\right)}\left(z^{0}\right)\right| \\
\leq C_{\varphi}^{\prime}\left\{\left(\left|x-x^{0}\right|^{2}+|\xi /| \xi\left|-\xi^{0}\right|^{2}\right)^{1 / 2}+\theta^{2} h^{2}\langle\xi\rangle_{h}^{-2}\right\},
\end{gathered}
$$


if $\theta \leq 1 / 2, \quad\left|x-x^{0}\right|^{2}+|\xi /| \xi\left|-\xi^{0}\right|^{2} \leq 1 / 2$ and $|\xi| \geq h$. Taking $\mathscr{C}$ and $\theta$ sufficiently small, $\Lambda_{h}(x, \xi)$ satisfies (4.9).

Q.E. D.

Lemma 4.4. Modifying a conic neighborhood $\mathscr{C}$ of $z^{0}$, there are symbols $W_{h}(x, \xi)(h \geq 1)$ and positive constants $C_{0}^{\prime}$ and $A_{0}^{\prime}$ such that

$$
\begin{aligned}
& W_{h}(x, \xi) \leq 2\langle\xi\rangle_{h}^{1 / \kappa}, \\
& \left|W_{h}^{(\alpha)}(x, \xi)\right| \leq C_{0}^{\prime} A_{0}^{\prime|\alpha|+|\beta|}(|\alpha|+|\beta|) !\langle\xi\rangle_{h}^{1 / \kappa-|\alpha|}, \\
& \langle\xi\rangle_{h}^{-2 / \kappa}\left\{|\xi|^{2}\left|\nabla_{\xi} W_{h}(x, \xi)\right|^{2}+\left|\nabla_{x} W_{h}(x, \xi)\right|^{2}\right\} \leq \varepsilon_{1}^{2} \\
& \quad \text { for }(x, \xi) \in \mathscr{C} \text { with }|\xi| \geq h, \\
& \varepsilon_{1}\langle\xi\rangle_{h}^{1 / \kappa} / 2+\left|\Lambda_{h}(x, \xi)\right|<W_{h}(x, \xi)+c_{1} h^{1 / \kappa} \text { for }|\xi| \geq h,
\end{aligned}
$$

where $\varepsilon_{1}(<2)$ and $c_{1}$ are the constants in Lemma 4.3.

Proof. There is a conic neighborhood $\tilde{\mathscr{C}}$ of $z^{0}$ such that $\mathscr{C} \Subset \tilde{\mathscr{C}}$ and

$$
\left|\Lambda_{h}(x, \xi)\right| \leq \varepsilon_{1}\langle\xi\rangle_{h}^{1 / x} / 8+c_{1} h^{1 / \kappa} \quad \text { for }(x, \xi) \in \tilde{\mathscr{C}} \text { with }|\xi| \geq h,
$$

modifying $\mathscr{C}$. Choose $w(x, \xi) \in C^{2}\left(T^{*} \boldsymbol{R}^{n}\right)$ so that the first and second order derivatives of $w$ are bounded, and $3 \varepsilon_{1} / 4 \leq w(x, \xi) \leq 2$, $w(x, \xi)$ $=3 \varepsilon_{1} / 4$ for $(x, \xi) \in \mathscr{C}$ with $|\xi| \geq 1 / 2$ and $w(x, \xi)=2$ for $(x, \xi) \notin \tilde{\mathscr{C}}$ with $|\xi| \geq 1 / 2$. Put $w_{j}(x, \xi)=E_{j} * w(x, \xi)$ and $W_{h}(x, \xi)=w_{j}\left(x, \xi /\langle\xi\rangle_{h}\right)$ $\times\langle\xi\rangle_{h}^{1 / \kappa}$, where $E_{j}(x, \xi)=j^{n}(4 \pi)^{-n} \exp \left[-j\left(|x|^{2}+|\xi|^{2}\right) / 4\right]$. If $j$ is sufficiently large, then $W_{h}(x, \xi)$ satisfies the conditions in the lemma.

Q.E. D.

We may assume that for any $A>0$ there is $C \equiv C_{A}>0$ (resp. there are $A>0$ and $C>0$ ) such that

$$
\left|p_{(\beta)}^{(\alpha)}(x, \xi)\right| \leq C A^{|\alpha|+|\beta|}(|\alpha|+|\beta|) ! \kappa\langle\xi\rangle^{m-|\alpha|}
$$

if $*=(\kappa)$ (resp. if $*=\{\kappa\})$. From (4.1) it follows that

$$
\operatorname{Re}\left\{\left(\bar{c}_{0} /\left|c_{0}\right|\right) p_{m}(x, \xi ; \vartheta, t)\right\} \geq\left|c_{0}\right| t^{l} / 2
$$

for $(x, \xi) \in \mathscr{U}$ and $t_{0} / 3 \leq t \leq t_{0}$, where $c_{0}=p_{m z^{0}}(-i \vartheta)\left(=p_{m}\left(x^{0}, \xi^{0} ; \vartheta, t\right)\right.$ $\left.\times t^{-l}\right)$, modifying $\mathscr{U}$ if necessary. Let $\mathscr{C}$ and $\mathscr{C}_{j}(j=1,2)$ be conic neighborhoods of $z^{0}$ such that $\mathscr{C} \cap\{|\xi|=1\} \Subset \mathscr{U}, \mathscr{C}_{1} \Subset \mathscr{C}_{2} \Subset \mathscr{C}$ and Lemmas 4.3 and 4.4 hold for $\mathscr{C}$. Let $\chi_{j}(x, \xi) \in \mathscr{E}^{\left({ }^{(x)}\right.}\left(T^{*} \boldsymbol{R}^{n} \backslash 0\right)(j=1,2)$ be positively homogeneous functions of degree 0 such that $0 \leq \chi_{j}(x, \xi)$ 
$\leq 1, \chi_{1}(x, \xi)=1$ on $\mathscr{C}_{3}$, supp $\chi_{1} \subset \mathscr{C}_{2}$ and supp $\chi_{2} \subset \mathscr{C}$, and let $\sigma(\xi) \in \mathscr{E}^{(\kappa)}$ be a function such that $0 \leq \sigma(\xi) \leq 1, \sigma(\xi)=1$ for $|\xi| \geq 1$ and $\sigma(\xi)=0$ for $|\xi| \leq 1 / 2$. We set for $h \geq 1$

$$
\begin{aligned}
& \tilde{p}_{m, h}(x, \xi)=\sigma_{h}(\xi) \chi_{2}(x, \xi) p_{m}\left(x, \xi ; \vartheta(\xi), t_{0}\left\{\left(1-\sigma_{2 h}(\xi)\right) h\right.\right. \\
& \left.\left.\quad+\left(1-\chi_{1}(x, \xi)\right)|\xi|\right\} / 3\right)+c_{0}\left(t_{0} / 3\right)^{l}|\xi|^{m} \sigma_{h}(\xi)\left(1-\chi_{2}(x, \xi)\right) / 2 \\
& \quad+c_{0}\left(t_{0} / 3\right)^{l} h^{m}\left(1-\sigma_{h}(\xi)\right), \\
& \tilde{p}_{h}(x, \xi)=\tilde{p}_{m, h}(x, \xi)+\sigma_{h}(\xi)\left(p(x, \xi)-p_{m}(x, \xi)\right),
\end{aligned}
$$

where $\sigma_{h}(\xi)=\sigma(\xi / h), \vartheta=\left(\vartheta_{x}, \vartheta_{\xi}\right)$ and $\vartheta(\xi)=\left(\vartheta_{x} /|\xi|, \vartheta_{\xi}\right)$. Note that $\tilde{p}_{m, h}(x, \xi)=p_{m}(x, \xi)$ and $\tilde{p}_{h}(x, \xi)=p(x, \xi)$ for $(x, \xi) \in \mathscr{C}_{1}$ with $|\xi| \geq 2 h$, and that $\tilde{p}_{h}(x, \xi)=\tilde{p}_{h^{\prime}}(x, \xi)$ if $h^{\prime} \geq h$ and $|\xi| \geq 2 h^{\prime}$.

Lemma 4.5. There are positive constants $C^{\prime} \equiv C_{x_{1}, x_{2}, \sigma_{A}}^{\prime}(C)$ and $d_{0}$ and $h_{0} \geq 1$ such that

$$
\left|\tilde{p}_{h(\beta)}^{(\alpha)}(x, \xi)\right| \leq C^{\prime}\left(2^{2} A\right)^{|\alpha|+\mid \beta_{1}}(|\alpha|+|\beta|) !^{\kappa}\langle\xi\rangle_{h}^{m-|\alpha|},
$$$$
\text { (4.13) }\left|\tilde{p}_{h}(x, \xi)\right| \geq d_{0}\langle\xi\rangle_{h}^{m} \quad \text { if }(x, \xi) \notin \mathscr{C}_{2} \text { or }|\xi| \leq h \text { and if } h \geq h_{0} \text {. }
$$

Proof. Since $\langle\xi\rangle \leq\langle\xi\rangle_{h} \leq \sqrt{5}\langle\xi\rangle$ for $h \geq 1$ and $|\xi| \geq h / 2$, (4.12) easily follows. If $|\xi| \leq h / 2$, then $\tilde{p}_{m, h}(x, \xi)=c_{0}\left(t_{0} / 3\right)^{l} h^{m}$. If $h / 2 \leq$ $|\xi| \leq h$, then $t_{0} / 3 \leq t_{0}\left\{h /|\xi|+\left(1-\chi_{1}(x, \xi)\right)\right\} / 3 \leq t_{0}$. Therefore, (4.11) gives

$$
\begin{aligned}
\operatorname{Re} & \left\{\left(\bar{c}_{0} /\left|c_{0}\right|\right) \tilde{p}_{m, h}(x, \xi)\right\} \geq\left|c_{0}\right|\left(t_{0} / 3\right)^{l}\left\{\left(h+\left(1-\chi_{1}(x, \xi)\right)|\xi|\right)\right. \\
& \times|\xi|^{m-l} \sigma_{h}(\xi) \chi_{2}(x, \xi) / 2+|\xi|^{m} \sigma_{h}(\xi)\left(1-\chi_{2}(x, \xi)\right) / 2 \\
& \left.+h^{m}\left(1-\sigma_{h}(\xi)\right)\right\} \geq 5^{-m_{+} / 2}\left|c_{0}\right|\left(t_{0} / 3\right)^{l}\langle\xi\rangle_{h}^{m} / 2 \quad \text { if } h / 2 \leq|\xi| \leq h,
\end{aligned}
$$

where $m_{+}=\max (m, 0)$. Since $t_{0} / 3 \leq t_{0}\left\{\left(1-\sigma_{2 h}(\xi)\right) h /|\xi|+1\right\} / 3 \leq t_{0}$ for $|\xi| \geq h$, it follows from (4.11) that

$$
\begin{aligned}
\operatorname{Re} & \left\{\left(\bar{c}_{0} /\left|c_{0}\right|\right) \tilde{p}_{m, h}(x, \xi)\right\} \geq\left|c_{0}\right|\left(t_{0} / 3\right)^{l} \\
& \times\left\{\left(\left(1-\sigma_{2 h}(\xi)\right) h+|\xi|\right)^{l}|\xi|^{m-l} \chi_{2}(x, \xi)+|\xi|^{m}\left(1-\chi_{2}(x, \xi)\right)\right\} / 2 \\
\geq & 2^{-1-m_{+} / 2}\left|c_{0}\right|\left(t_{0} / 3\right)^{l}\langle\xi\rangle_{h}^{m} \quad \text { if }|\xi| \geq h \text { and }(x, \xi) \notin \mathscr{C}_{2} .
\end{aligned}
$$

So there is $d_{0}>0$ such that

$$
\left|\tilde{p}_{m, h}(x, \xi)\right| \geq 2 d_{0}\langle\xi\rangle_{h}^{m} \quad \text { if }(x, \xi) \notin \mathscr{C}_{2} \text { or }|\xi| \leq h .
$$

This proves (4.13).

Q.E. D.

Applying Proposition 2. 13, we can write 
$\exp \left[a \Lambda_{h}^{b}\right](x, D) \tilde{p}_{h}(x, D)^{R} \exp \left[-a \Lambda_{h}^{b}\right](x, D)=\tilde{p}_{h}^{a, b}(x, D)+r_{h}^{a, b}(x, D)$ if $0 \leq a \leq \hat{a}_{0} A^{-1 / \hbar}$ and $-1 \leq b \leq 1$, where $\Lambda_{h}^{b}(x, \xi)=\Lambda_{h}(x, \xi)+b W_{h}(x, \xi)$, $\hat{a}_{0}>0, \hat{\varepsilon}>0$ and

$$
\begin{aligned}
\left|\hat{p}_{h(\beta)}^{a, b(\alpha)}(x, \xi)\right| \leq & C_{A}(C)\left(2^{2+2 \kappa} A\right)^{|\alpha|+|\beta|}(|\alpha|+|\beta|) !^{\kappa} \\
& \times\langle\xi\rangle_{h}^{m-(1-1 / \kappa)(l+1)-|\alpha|} \\
\hat{p}_{h}^{a, b}(x, \xi)= & \tilde{p}_{h}^{a, b}(x, \xi)-\sum_{|\alpha|+|\beta| \leq l}(\alpha ! \beta !)^{-1} \\
& \times\left\{\tilde{p}_{h(\beta)}(x, \xi) \omega^{\beta}\left(a \Lambda_{h}^{b} ; x, \xi\right) \omega_{\alpha}\left(-a \Lambda_{h}^{b} ; x, \xi\right)\right\}^{(\alpha)}, \\
\left|r_{h(\beta)}^{a, b(\alpha)}(x, \xi)\right| & \leq C_{A}(|\alpha|, C)\left(2^{2+4 \kappa} A\right)^{|\beta|}|\beta| !^{\kappa} \exp \left[-\hat{\varepsilon} A^{-1 / \kappa}\langle\xi\rangle_{h}^{1 / \kappa}\right] .
\end{aligned}
$$

Lemma 4.6. For a fixed $N_{0}$ there are positive constants $a_{p}, h_{p}(a)$, $C_{A}(C), C_{p}, c$ and $c_{1}$ such that $\tilde{p}_{h}^{a, b}(x, \xi)$ satisfies the condition ( $H$; $\left.C_{A}(C), c_{1} A, c a^{l}, C_{p}, a^{-1}, N_{0}\right)$ with $m^{\prime}=m-(1-1 / k) l, \delta=1-1 / \kappa$ and $\rho=1 / \kappa$ defined in $\S 3$ if $0<a \leq \hat{a}_{0} A^{-1 / \kappa},-1 \leq b \leq 1$ and $h \geq h_{p}(a)$, and if $a \geq a_{p}$ when $\kappa=\kappa_{0}$. Here $a_{p}, h_{p}(a)$ and $C_{p}$ do not depend on the choice of $A$ when $* 1=\left(\kappa_{1}\right)$.

Proof. When $(x, \xi) \notin \mathscr{C}_{2}$ or $|\xi| \leq h$, by Lemma 4.5 we can apply Lemma 3.1 with $\delta=(1-1 / \kappa) / 2$ and $\rho=\delta+1 / \kappa$. So it is sufficient to estimate $\tilde{p}_{h}^{a, b}(x, \xi)$ for $(x, \xi) \in \mathscr{C}_{2}$ with $|\xi| \geq h$. Let $(x, \xi) \in \mathscr{C}_{2}$ and $|\xi| \geq h$. Then we have

$$
\begin{aligned}
& \tilde{p}_{h}^{a, b}(x, \xi)=p_{m, h}^{a, b}(x, \xi)+s_{h, 1}^{a, b}(x, \xi)+s_{h, 2}^{a, b}(x, \xi), \\
& \tilde{p}_{m, h}(x, \xi)=p_{m}\left(x, \xi ; \vartheta(\xi), d_{1}(x, \xi)\right),
\end{aligned}
$$

where

$$
\begin{aligned}
p_{m, h}^{a, b}(x, \xi)= & \sum_{|\alpha|+|\beta| \leq l}(\alpha ! \beta !)^{-1} \tilde{p}_{m, h(\beta)}^{(\alpha)}(x, \xi) \\
& \times\left(i a \nabla_{x} \Lambda_{h}^{b}(x, \xi)\right)^{\alpha}\left(a \nabla_{\xi} \Lambda_{h}^{b}(x, \xi)\right)^{\beta} \\
s_{h, 1}^{a, b}(x, \xi)= & \sum_{|\alpha|+|\beta| \leq l}(\alpha ! \beta !)^{-1} \\
& \times\left\{\tilde{p}_{m, h(\beta)}(x, \xi) \omega^{\beta}\left(a \Lambda_{h}^{b} ; x, \xi\right) \omega_{\alpha}\left(-a \Lambda_{h}^{b} ; x, \xi\right)\right\}^{(\alpha)} \\
& \quad-p_{m, h}^{a, b}(x, \xi) \\
s_{h, 2}^{a, b}(x, \xi)= & \hat{p}_{h}^{a, b}(x, \xi)+\sum_{|\alpha|+|\beta| \leq l}(\alpha ! \beta !)^{-1}\left\{\left(p_{(\beta)}(x, \xi)\right.\right. \\
& \left.\left.-p_{m(\beta)}(x, \xi)\right) \omega^{\beta}\left(a \Lambda_{h}^{b} ; x, \xi\right) \omega_{\alpha}\left(-a \Lambda_{h}^{b} ; x, \xi\right)\right\}^{(\alpha)} \\
d_{1}(x, \xi)= & t_{0}\left\{\left(1-\sigma_{2 h}(\xi)\right) h+\left(1-\chi_{1}(x, \xi)\right)|\xi|\right\} / 3 .
\end{aligned}
$$

Put

$$
\begin{aligned}
& \tilde{p}_{m, h}^{a, b}(x, \xi)=p_{m}\left(x-X(x, \xi), \xi-\Xi(x, \xi) ; v_{x}(x, \xi), v_{\xi}(x, \xi), d(x, \xi)\right) \\
& X(x, \xi)=a\left\{\Lambda_{h}^{b}(x, \xi), d_{1}(x, \xi) /|\xi|\right\} \vartheta_{x}
\end{aligned}
$$




$$
\begin{aligned}
& \Xi(x, \xi)=a\left\{\Lambda_{h}^{b}(x, \xi), d_{1}(x, \xi)\right\} \vartheta_{\xi}, \\
& v_{x}(x, \xi)=d(x, \xi)^{-1}\left\{d_{1}(x, \xi) \vartheta_{x} /|\xi|+a \nabla_{\xi} \Lambda_{h}^{b}(x, \xi)\right\}, \\
& v_{\xi}(x, \xi)=d(x, \xi)^{-1}\left\{d_{1}(x, \xi) \vartheta_{\xi}-a \nabla_{x} \Lambda_{h}^{b}(x, \xi)\right\}, \\
& d(x, \xi)=d_{1}(x, \xi)+a\langle\xi\rangle_{h}^{1 / x},
\end{aligned}
$$

where $\{f, g\}=\sum_{j=1}^{n}\left\{\left(\partial f / \partial \xi_{j}\right)\left(\partial g / \partial x_{j}\right)-\left(\partial f / \partial x_{j}\right)\left(\partial g / \partial \xi_{j}\right)\right\}$. Note that there is $h(a)>0$ such that $(x-X(x, \xi), \xi-\Xi(x, \xi)) \in \mathscr{C}$ if $h \geq h(a)$ and $-1 \leq b \leq 1,\left((x, \xi) \in \mathscr{C}_{2}\right.$ and $\left.|\xi| \geq h\right)$. From Lemmas 4.3 and 4.4 it follows that $\left(|\xi| v_{x}(x, \xi), v_{\xi}(x, \xi)\right) \in M_{1}$. Therefore, by Corollary of Lemma 4.2 we have

$$
\begin{aligned}
& \left|\tilde{p}_{m, h}^{a, b}(x, \xi)\right| \geq c d(x, \xi)^{l}|\xi|^{m-l}, \\
& \left|p_{m(\beta)}^{(\alpha)}(x-X(x, \xi), \xi-\Xi(x, \xi)) / \tilde{p}_{m, h}^{a, b}(x, \xi)\right| \\
& \leq C(\alpha, \beta) d(x, \xi)^{-|\alpha|-|\beta|}|\xi|^{|\beta|}
\end{aligned}
$$

if $h \geq h(a)$, modifying $h(a)$. A simple calculation gives

$$
\begin{aligned}
& \left|p_{m(\beta)}^{(\alpha)}(x, \xi) / \tilde{p}_{m, h}^{a, b}(x, \xi)\right| \leq C^{\prime \prime} d(x, \xi)^{-|\alpha|-|\beta|}\langle\xi\rangle_{h}^{|\beta|} \\
& \leq C^{\prime \prime} a^{-|\alpha|-|\beta|}\langle\xi\rangle_{h}^{(1-1 / \kappa)|\beta|-|\alpha| / \kappa} \quad \text { if } h \geq h_{p}(a) \text { and }|\alpha|+|\beta| \leq l \text {, }
\end{aligned}
$$

where $h_{p}(a)>0$. It is easy to see that

$$
\begin{aligned}
& \sum_{|\tilde{\alpha}|+|\tilde{\beta}| \leq l}(\tilde{\alpha} ! \tilde{\beta} !)^{-1}\left\{p_{m(\beta)}^{(\alpha)}(x, \xi)\left(d_{1}(x, \xi) \vartheta_{x} /|\xi|\right)^{\beta}\right.
\end{aligned}
$$

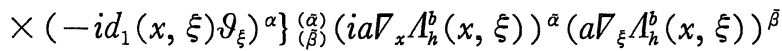

$$
\begin{aligned}
& =\sum_{\left|\alpha^{0}\right|+\left|\beta^{0}\right| \leq l-N}\left(\alpha^{0} ! \beta^{0 !}\right)^{-1} p_{m\left(\beta+\beta^{0}\right)}^{\left(\alpha+\alpha^{0}\right)}(x, \xi) \\
& \times\left(i a \nabla_{x} \Lambda_{h}^{b}(x, \xi)\right)^{\alpha^{0}}\left(a \nabla_{\xi} \Lambda_{h}^{b}(x, \xi)\right)^{\beta^{0}}\left(-i d_{1}(x, \xi) \vartheta_{\xi}-\Xi(x, \xi)\right)^{\alpha} \\
& \times\left(d_{1}(x, \xi) \vartheta_{x} /|\xi|-i X(x, \xi)\right)^{\beta}+\Sigma_{1} \text {, } \\
& \Sigma_{1}=\left(\sum_{\left|\alpha^{0}\right|+\cdots+\left|\alpha^{N}\right|+\left|\beta^{0}\right|+\cdots+\left|\beta^{N}\right| \leq l}\right. \\
& \left.-\sum_{\left|\alpha^{0}\right|+\left|\beta^{0}\right| \leq l-N,\left|\alpha^{j}\right|+\left|\beta^{j}\right| \leq 1,1 \leq j \leq N}\right)\left(\alpha^{0} ! \beta^{0 !} !\right)^{-1} p_{m\left(\beta+\beta^{0}\right)}^{\left(\alpha+\alpha^{0}\right)}(x, \xi) \\
& \times\left(i a \nabla_{x} \Lambda_{h}^{b}(x, \xi)\right)^{\alpha^{0}}\left(a \nabla_{\xi} \Lambda_{h}^{b}(x, \xi)\right)^{\beta^{0}} \Pi_{j=1}^{|\alpha|}\left(\alpha^{j !} ! \beta^{\prime} !\right)^{-1} d_{1\left(\beta^{j}\right)}^{\left(\alpha^{j}\right)}(x, \xi) \\
& \times\left(i a \nabla_{x} \Lambda_{h}^{b}(x, \xi)\right)^{\alpha^{j}}\left(a \nabla_{\xi} \Lambda_{h}^{b}(x, \xi)\right)^{\beta^{j}} \Pi_{j=|\alpha|+1}^{N}\left(\alpha^{j} ! \beta^{\jmath} !\right)^{-1} \\
& \times\left(d_{1}(x, \xi) /|\xi|\right)_{\left(\beta^{j}\right)}^{\left(\alpha^{j}\right)}\left(i a \nabla_{x} \Lambda_{h}^{b}(x, \xi)\right)^{\alpha^{j}}\left(a \nabla_{\xi} \Lambda_{h}^{b}(x, \xi)\right)^{\beta^{j}}\left(-i \vartheta_{\xi}\right)^{\alpha} \vartheta_{x}^{\beta} \text {, }
\end{aligned}
$$

where $N=|\alpha|+|\beta| \leqq l$. Then we have

$$
\begin{aligned}
& \left|\Sigma_{1} / \tilde{p}_{m, h}^{a, b}(x, \xi)\right| \\
& \leq C_{p}\left(\sum_{\left|\alpha^{0}\right|+\left|\beta^{0}\right| \leq l-N, 1 \leq\left|\alpha^{1}\right|+\cdots+\left|\beta^{N}\right| \leq N+1} a^{\left|\alpha^{0}\right|+\cdots+\left|\beta^{N}\right|}\right. \\
& \times d(x, \xi)^{-N-\left|\alpha^{0}\right|-\left|\beta^{0}\right|} d_{1}(x, \xi)^{N-\left(\left|\alpha^{1}\right|+\cdots+\left|\beta^{N}\right|-1\right)} \\
& \times\langle\xi\rangle\rangle_{h}^{\left(\left|\alpha^{0}\right|+\cdots+\left|\alpha^{N}\right|\right) / \kappa+\left(\left|\beta^{0}\right|+\cdots+\left|\beta^{N}\right|\right) / \kappa-1} \\
& +\sum_{\left|\alpha^{0}\right|+\left|\beta^{0}\right| \leq l-N,\left|\alpha^{1}\right|+\cdots+\left|\beta^{N}\right|>N+1} a^{\left|\alpha^{0}\right|+\cdots+\left|\beta^{N}\right|} \\
& \times d(x, \xi)^{-N-\left|\alpha^{0}\right|-\left|\beta^{0}\right|}\langle\xi\rangle_{h}^{|\beta|+\left|\beta^{0}\right|+\left(\left|\alpha^{0}\right|+\cdots+\left|\alpha^{N}\right|\right) / \kappa}
\end{aligned}
$$




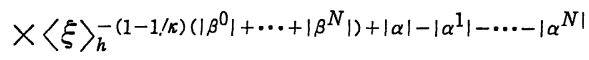

$$
\begin{aligned}
& +\sum_{l-N<\left|\alpha^{0}\right|+\left|\beta^{0}\right| \leq l,\left|\alpha^{1}\right|+\cdots+\left|\beta^{N}\right| \leq N-1} a^{\left|\alpha^{0}\right|+\cdots+\left|\beta^{N}\right|} \\
& \times d(x, \xi)^{-l} d_{1}\left(x_{1}, \xi\right)^{N-\left(\left|\alpha^{1}\right|+\cdots+\left|\beta^{N}\right|\right)} \\
& \left.\times\langle\xi\rangle_{h}^{l-N-(1-1 / \kappa)\left(\left|\alpha^{0}\right|+\left|\beta^{0}\right|\right)+\left(\left|\alpha^{1}\right|+\cdots+\left|\beta^{N}\right|\right) / \kappa}\right\} \\
& \leq a C_{p}^{\prime}\langle\xi\rangle_{h}^{1 / \kappa-1} \text { if } h \geq h_{p}(a) \text {, }
\end{aligned}
$$

modifying $h_{p}(a)$. Therefore, we have

$$
\begin{aligned}
& p_{m, h}^{a, b}(x, \xi)=\sum_{\left|\alpha^{1}\right|+\left|\alpha^{2}\right|+\left|\beta^{1}\right|+\left|\beta^{2}\right| \leq l}\left(\alpha^{1} ! \alpha^{2} ! \beta^{1} ! \beta^{2} !\right)^{-1} \\
& \times p_{m\left(\beta^{1}+\beta^{2}\right)}^{\left(\alpha^{1}+\alpha^{2}\right)}(x, \xi)(-\Xi(x, \xi))^{\alpha^{1}}(-i X(x, \xi))^{\beta^{1}}\left(-i d(x, \xi) v_{\xi}(x, \xi)\right)^{\alpha^{2}} \\
& \times\left(d(x, \xi) v_{x}(x, \xi)\right)^{\beta^{2}}+\Sigma_{2}=\tilde{p}_{m, h}^{a, b}(x, \xi)+\Sigma_{2}+\Sigma_{3},
\end{aligned}
$$

where $\left|\Sigma_{2} / \tilde{p}_{m, h}^{a, b}(x, \xi)\right| \leq a C_{p}^{\prime \prime}\langle\xi\rangle_{h}^{1 / \kappa-1}$ if $h \geq h_{p}(a)$ and

$$
\begin{aligned}
\Sigma_{3} & =\sum_{|\alpha|+|\beta| \leq l}(\alpha ! \beta !)^{-1}\left\{p_{m(\beta)}^{(\alpha)}(x-X(x, \xi), \xi-\Xi(x, \xi))\right. \\
& -\sum_{|\tilde{\alpha}|+|\bar{\beta}| \leq l-|\alpha|-|\beta|}(\tilde{\alpha} ! \tilde{!} !)^{-1} p_{m(\beta+\tilde{\beta})}^{(\alpha+\tilde{\alpha})}(x, \xi)(-\Xi(x, \xi))^{\tilde{\alpha}} \\
& \left.\times(-i X(x, \xi))^{\bar{\beta}}\right\}\left(-i d(x, \xi) v_{\xi}(x, \xi)\right)^{\alpha}\left(d(x, \xi) v_{x}(x, \xi)\right)^{\beta} .
\end{aligned}
$$

It follows from (4.14) that $\left|\Sigma_{3} / \tilde{p}_{m, h}^{a, b}(x, \xi)\right| \leq a C_{p}^{\prime \prime \prime}\langle\xi\rangle_{h}^{1 / \kappa-1}$ if $h \geq h_{p}(a)$. So we have

$$
\left|p_{m, h}^{a, b}(x, \xi)-\tilde{p}_{m, h}^{a, b}(x, \xi)\right| /\left|\tilde{p}_{m, h}^{a, b}(x, \xi)\right| \leq 1 / 6
$$

if $h \geq h_{p}(a)$. The same argument as in (4.15) yields

$$
\left|\tilde{p}_{m, h(\beta)}^{(\alpha)}(x, \xi) / \tilde{p}_{m, h}^{a, b}(x, \xi)\right| \leq C_{p} a^{-|\alpha|-|\beta|}\langle\xi\rangle_{h}^{(1-1 / \kappa)|\beta|-|\alpha| / \kappa}
$$

if $h \geq h_{p}(a)$ and $|\alpha|+|\beta| \leq l$. This implies that

$$
\left|s_{h, 1}^{a, b}(x, \xi) / \tilde{p}_{m, h}^{a, b}(x, \xi)\right| \leq 1 / 6 \quad \text { if } h \geq h_{p}(a),
$$

since

$$
\begin{gathered}
\left|\left\{\omega_{\alpha}\left(-a \Lambda_{h}^{b} ; x, \xi\right)-\left(i a D_{x} \Lambda_{h}^{b}(x, \xi)\right)^{\alpha}\right\}_{(\tilde{\alpha})}^{(\tilde{\alpha})}\right| \leq C(\alpha, \tilde{\alpha}, \tilde{\beta}, a) \\
\times\langle\xi\rangle_{h}^{|\alpha| / \kappa-1 / \kappa-|\tilde{\alpha}|}, \\
\left|\left\{\omega^{\beta}\left(a \Lambda_{h}^{b} ; x, \xi\right)-\left(a D_{\xi} \Lambda_{h}^{b}(x, \xi)\right)^{\beta}\right\}_{(\tilde{\beta})}^{(\tilde{\alpha})}\right| \leq C(\beta, \tilde{\alpha}, \tilde{\beta}, a) \\
\times\langle\xi\rangle_{h}^{-(1-1 / \kappa)|\beta|-1 / k-|\tilde{\alpha}|} \\
\left|\left\{\omega^{\beta}\left(a \Lambda_{h}^{b} ; x, \xi\right) \omega_{\alpha}\left(-a \Lambda_{h}^{b} ; x, \xi\right)\right\}_{(\tilde{\beta})}^{(\tilde{\alpha})}\right| \leq C(\alpha, \beta, \tilde{\alpha}, \tilde{\beta}, a) \\
\times\langle\xi\rangle_{h}^{|\alpha| / \kappa-(1-1 / \kappa)|\beta|-|\tilde{\alpha}|} .
\end{gathered}
$$

It is obvious that

$$
\left|s_{h, 2}^{a, b}(x, \xi) / \tilde{p}_{m, h}^{a, b}(x, \xi)\right| \leq 1 / 6 \quad \text { if } h \geq h_{p}(a) \text { and } \kappa<\kappa_{0} .
$$

When $\kappa=\kappa_{0}$, we have also

$$
\left|s_{h, 2}^{a, b}(x, \xi) / \tilde{p}_{m, h}^{a, b}(x, \xi)\right| \leq 1 / 6 \quad \text { if } h \geq h_{p}(a) \text { and } a \geq a_{p},
$$


where $a_{p}>0$. Thus we have

$$
\left|\tilde{p}_{h}^{a, b}(x, \xi)\right| \geq\left|\tilde{p}_{m, h}^{a, b}(x, \xi)\right| / 2 \geq c^{\prime} a^{l}\langle\xi\rangle_{h}^{m-(1-1 / \kappa) l}
$$

if $h \geq h_{p}(a)$, and if $a \geq a_{p}$ when $\kappa=\kappa_{0}$, where $c^{\prime}>0$. Similarly, we have

$$
\begin{aligned}
& \left|\tilde{p}_{h(\beta)}^{a, b(\alpha)}(x, \xi) / \tilde{p}_{h}^{a, b}(x, \xi)\right| \leq 2\left|\tilde{p}_{h(\beta)}^{a, b(\alpha)}(x, \xi) / \tilde{p}_{m, h}^{a, b}(x, \xi)\right| \\
& \leq C_{p}(\alpha, \beta) a^{-|\alpha|-|\beta|}\langle\xi\rangle_{h}^{(1-1 / \kappa)|\beta|-|\alpha| / \kappa}
\end{aligned}
$$

if $h \geq h_{p}(a)$, and if $a \geq a_{p}$ when $\kappa=\kappa_{0}$. Here we have modified $h_{p}(a)$, if necessary. This proves the lemma.

Q.E. D.

From Proposition 3.4 (or its proof) and Lemma 4.6 it follows that $\tilde{p}_{h}^{a, b}(x, D)+r_{h}^{a, b}(x, D)$ has the inverse $\tilde{Q}_{h}^{a, b}$, i, e., $\tilde{Q}_{h}^{a, b}$ maps continuously $L^{2}$ to $H^{m-(1-1 / \kappa) l}\left(\subset H^{m}\right)$ and $H^{(1-1 / k) l}$ to $H^{m}$, and satisfies $\tilde{Q}_{h}^{a, b}\left(\tilde{p}_{h}^{a, b}(x, D)+r_{h}^{a, b}(x, D)\right)=I$ on $H^{m}$ and $\left(\tilde{p}_{h}^{a, b}(x, D)+r_{h}^{a, b}(x, D)\right) \tilde{Q}_{h}^{a, b}$ $=I$ on $H^{(1-1 / \kappa) l}$ if $a, b$ and $h$ satisfy the following conditions;

(4. 16) $0<a \leq \hat{a}_{0} A^{-1 / \kappa},-1 \leq b \leq 1$ and $h \geq h_{p, a}$, and $a \geq a_{p}^{\prime}$ when $\kappa=\kappa_{0}$,

where $h_{p, a}$ and $a_{p}^{\prime}$ are positive constants. By Lemma 2.14 and Proposition 3.4, for any $\varepsilon>0$ there is $h_{a}(\varepsilon)>0$ such that $1+q_{h}^{a, b}(x, D)$ $\equiv{ }^{R} \exp \left[-a \Lambda_{h}^{b}\right](x, D) \exp \left[a \Lambda_{h}^{b}\right](x, D)$ has the inverse $\left(1+q_{h}^{a, b}(x, D)\right)^{-1}$ which maps continuously $L_{\kappa, \varepsilon^{\prime}}^{2}$ to $L_{\kappa, \varepsilon^{\prime}}^{2}$ if $\left|\varepsilon^{\prime}\right| \leq \varepsilon, h \geq h_{a}(\varepsilon), a \geq 0$ and $-1 \leq b \leq 1$. Let us introduce the following spaces.

Definition 4.7. Let $\Lambda(x, \xi)$ satisfy (2.13). For $s \in \boldsymbol{R}$ we define $H_{\Lambda}^{s}=\left\{f ;\left(e^{\Lambda}\right)(x, D) f \in H^{s}\right\}$, and write $L_{\Lambda}^{2}=H_{\Lambda}^{0}, H_{a, b}^{s}=H_{a \Lambda_{h}^{b}}^{s}$ and $L_{a, b}^{2}$ $=H_{a, b}^{0}$.

Lemma 4.8. (i) $f \in H^{s}$ if and only if ${ }^{R}\left(e^{-\Lambda}\right)(x, D) f \in H_{\Lambda}^{s}$. (ii) If $f \in H_{a \Lambda}^{s}$ and $k(x, \xi)$ satisfies

$$
\left|k_{(\beta)}^{(\alpha)}(x, \xi)\right| \leq C_{1} A_{1}^{|\alpha|+|\beta|}(|\alpha|+|\beta|) !^{\kappa}\langle\xi\rangle_{h}^{m-|\alpha|},
$$

then $k(x, D) f \in H_{a \Lambda}^{s-m}$ for $|a| \leq c_{\Lambda} A_{1}^{-1 / \kappa}$, where $c_{\Lambda}>0$.

Proof. By Lemma 2.14 we can write

$$
\begin{gathered}
R\left(e^{-a \Lambda}\right)(x, D)\left(e^{a \Lambda}\right)(x, D)=1+q_{h^{\prime}}^{a}(x, D)+r_{h^{\prime}}^{a}(x, D), \\
\left(e^{a \Lambda}\right)(x, D)^{R}\left(e^{-a \Lambda}\right)(x, D)=1+\tilde{q}_{h^{\prime}}^{a}(x, D)+\tilde{r}_{h^{\prime}}^{a}(x, D),
\end{gathered}
$$

where $h^{\prime} \geq 1$ and

$$
\left|q_{h^{\prime}(\beta)}^{a(\alpha)}(x, \xi)\right| \leq C_{a, \Lambda, d} d^{|\alpha|+|\beta|}(|\alpha|+|\beta|) !^{\kappa}\langle\xi\rangle_{h}^{1 / \kappa-1-|\alpha|} \quad \text { for any } d>0,
$$




$$
\begin{aligned}
& q_{h^{\prime}}^{a}(x, \xi)=0 \quad \text { for }|\xi| \leq h^{\prime}, \\
& r_{h^{\prime}}^{a}(x, D): L_{\kappa, \varepsilon}^{2} \rightarrow L_{\kappa, \varepsilon^{\prime}}^{2} \quad \text { for any } \varepsilon, \varepsilon^{\prime} \in \boldsymbol{R},
\end{aligned}
$$

and $\tilde{q}_{h^{\prime}}^{a}(x, \xi)$ and $\tilde{r}_{h^{\prime}}^{a}(x, \xi)$ have the same properties as $q_{h^{\prime}}^{a}(x, \xi)$ and $r_{h^{\prime}}^{a}(x, \xi)$, respectively. From Proposition 3.4 it follows that $1+q_{h^{\prime}}^{a}$ $(x, D)$ and $1+\tilde{q}_{h^{\prime}}^{a}(x, D)$ have the inverses $\left(1+q_{h^{\prime}}^{a}(x, D)\right)^{-1}$ and $\left(1+\tilde{q}_{h^{\prime}}^{a}(x, D)\right)^{-1}$, respectively, which map continuously $L_{\kappa, \varepsilon}^{2}$ to $L_{\kappa, \varepsilon}^{2}$ and $H_{\kappa, \varepsilon}^{s}$ to $H_{\kappa, \varepsilon}^{s}$, if $h^{\prime}$ is large enough and $|\varepsilon| \leq A_{1}^{-1 / \kappa}$. Then the assertion (i) is obvious. Using (2.29), we have

$$
\begin{aligned}
& \left(e^{a \Lambda}\right)(x, D) k(x, D) f=e^{a \Lambda} k\left[{ }^{R}\left(e^{-a \Lambda}\right)\left(1+\tilde{q}_{h^{\prime}}^{a}\right)^{-1} e^{a \Lambda}\right. \\
& -\left(1+q_{h^{\prime}}^{a}\right)^{-1}\left\{{ }^{R}\left(e^{-a \Lambda}\right) \tilde{r}_{h^{\prime}}^{a}-r_{h}^{a} \cdot{ }^{R}\left(e^{-a \Lambda}\right)\right\}\left(1+\tilde{q}_{h^{\prime}}^{a}\right)^{-1} e^{a \Lambda} \\
& \left.-\left(1+q_{h^{\prime}}^{a}\right)^{-1} r_{h^{\prime}}^{a}\right] f
\end{aligned}
$$

if $f \in H_{a \Lambda}^{S}$ and $|a| \leq c_{\Lambda} A_{1}^{-1 / \kappa}$, where $c_{\Lambda}>0$. Here we have chosen $h^{\prime}$ sufficiently large according to $A_{1}$. Modifying $c_{\Lambda}$ if necessary, Propositions 2.12 and 2.13 imply that $\left(e^{a \Lambda}\right)(x, D) k(x, D)^{R}\left(e^{-a \Lambda}\right)(x, D)$ maps continuously $H^{s}$ to $H^{s-m}$ for $|a| \leq c_{A} A_{1}^{-1 / \kappa}$. This proves the assertion (ii)。

Q. E. D.

Lemma 4.9. Let $\Lambda(x, \xi)$ and $\Lambda^{\prime}(x, \xi)$ satisfy (2.13), where $h \geq 1$, and assume that a symbol $q(x, \xi)$ satisfies

$$
\left|q_{(\beta)}^{(\alpha)}(x, \xi)\right| \leq C_{d} d^{|\alpha|+|\beta|}|\alpha| !^{\kappa}|\beta| !^{\kappa}\langle\xi\rangle^{-|\alpha|} \text { for any } d>0 \text {. }
$$

If $\inf _{L>0} \sup _{(x, \xi) \in \operatorname{supp} q(x, \xi),|\xi| \geq L}\left(\Lambda(x, \xi)-\Lambda^{\prime}(x, \xi)\right)\langle\xi\rangle_{h}^{-1 / \kappa}<\varepsilon$, then $q(x, D)$ $\left(e^{\Lambda}\right)(x, D){ }^{R}\left(e^{-\Lambda^{\prime}}\right)(x, D) f$ and $\left(e^{\Lambda}\right)(x, D) q(x, D){ }^{R}\left(e^{-\Lambda^{\prime}}\right)(x, D) f$ belong to $L_{\kappa, a-\varepsilon}^{2}$ for $f \in L_{\kappa, a}^{2}$.

Proof. By Proposition 2.8 we can write $q(x, D)\left(e^{\Lambda}\right)(x, D)=$ $\tilde{q}(x, D)+\tilde{r}(x, D), \quad$ where $\quad \tilde{q}(x, \xi)=\sum_{j=0}^{\infty} \sum_{|\alpha|=j} \alpha !^{-1} \varphi_{j}^{R}(\xi) q^{(\alpha)}(x, \xi) \times$ $\left(e^{\Lambda(x, \xi)}\right)_{(\alpha)}$ and $\tilde{r}(x, D): L_{\kappa, a}^{2} \rightarrow L_{\kappa, a^{\prime}}^{21}$ continuously for any $a, a^{\prime} \in \boldsymbol{R}$. It follows from Corollary of Lemma 2.9 that

$$
\begin{aligned}
\left|\tilde{q}_{(\beta)}^{(\alpha)}(x, \xi)\right| & \leq C_{\Lambda, \rho, d} d^{|\alpha|+|\beta|}|\alpha| !^{\kappa}|\beta| !^{\kappa}\langle\xi\rangle^{-|\alpha|} \\
& \times \exp \left[\rho\langle\xi\rangle^{1 / \kappa}+\Lambda(x, \xi)\right] \text { for any } d, \rho>0,
\end{aligned}
$$

and that $\operatorname{supp} \tilde{q}(x, \xi) \subset \operatorname{supp} q(x, \xi)$. Since $\Lambda(x, \xi)$ and $\Lambda^{\prime}(x, \xi)$ satisfy (2.13), there are $c_{1}>0$ and $c_{2}>0$ such that $\inf _{L>0} \sup \{(\Lambda(x, \xi+$ $\left.\eta)-\Lambda^{\prime}(x+y, \xi+\eta)\right)\langle\xi\rangle^{-1 / \kappa} ;(x, \xi+\eta) \in \operatorname{supp} q,|\xi| \geq L,|\eta| \leq c_{1}\langle\xi\rangle$ and $\left.|y| \leq c_{2}\right\}<\varepsilon$. Applying Lemma 2.15 to $\tilde{q}(x, \xi)^{R}\left(e^{-\Lambda^{\prime}}\right)(x, D)$, we have $\tilde{q}(x, D)^{R}\left(e^{-\Lambda^{\prime}}\right)(x, D) f \in L_{\kappa, a-\varepsilon}^{2}$ for $f \in L_{\kappa, a}^{2}$, which proves that $q(x, D)$ 
$\times\left(e^{\Lambda}\right)(x, D)^{R}\left(e^{-\Lambda^{\prime}}\right)(x, D) f \in L_{\kappa, a-\varepsilon}^{2}$ for $f \in L_{\kappa, a}^{2}$. Similarly, we can prove $\left(e^{\Lambda}\right)(x, D) q(x, D){ }^{R}\left(e^{-\Lambda^{\prime}}\right)(x, D) f \in L_{\kappa, a-\varepsilon}^{2}$ for $f \in L_{\kappa, a}^{2}$.

Q. E. D

Let us construct parametrices of (MCP). Define $Q_{h}^{a, b}$ by

$$
Q_{h}^{a, b} f={ }^{R} \exp \left[-a \Lambda_{h}^{b}\right](x, D) \tilde{Q}_{h}^{a, b} \exp \left[a \Lambda_{h}^{b}\right](x, D) f .
$$

Then $Q_{h}^{a, b}$ maps continuously $L_{a, b}^{2}$ to $H_{a, b}^{m-(1-1 / \kappa) l}$ and $H_{a, b}^{(1-1 / \kappa) l}$ to $H_{a, b}^{m}$ and satisfies $Q_{h}^{a, b} \tilde{p}_{h}(x, D)=I$ on $H_{a, b}^{m}$ and $\tilde{p}_{h}(x, D) Q_{h}^{a, b}=I$ on $H_{a, b}^{(1-1 / \kappa) l}$ if $a, b$ and $h$ satisfy (4.16). In fact, by Lemma 2.14 and Proposition 3.4 we may assume that $1+\tilde{q}_{h}^{a, b}(x, D) \equiv \exp \left[a \Lambda_{h}^{b}\right](x, D){ }^{R} \exp \left[-a \Lambda_{h}^{b}\right]$ $(x, D)$ has the inverse $\left(1+\tilde{q}_{h}^{a, b}(x, D)\right)^{-1}$ which maps $H_{\kappa, \varepsilon}^{s}$ to $H_{\kappa, \varepsilon}^{s}$ if $|s| \leq|m|+(1-1 / \kappa) l,|\varepsilon| \leq 4 a,-1 \leq b \leq 1$ and $h \geq h_{p, a}$. We may also assume that $\left(1+q_{h}^{a, b}(x, D)\right)^{-1}$ maps continuously $L_{\kappa, \varepsilon}^{2}$ to $L_{\kappa, \varepsilon}^{2}$ if $|\varepsilon| \leq 4 a$, $-1 \leq b \leq 1$ and $h \geq h_{p, a}$. From Corollary of Lemma 2.9 and Proposition 2.12 it follows taht ${ }^{R} \exp \left[-a \Lambda_{h}^{b}\right](x, D) g \in L_{\kappa,-4 a}^{2}$ for $g \in H^{s}$, where $s \in \boldsymbol{R}$, $a>0,-1 \leq b \leq 1$ and $h \geq h_{p, a}$. So, by (2.29) we have

(4. 17) $f={ }^{R} \exp \left[-a \Lambda_{h}^{b}\right](x, D)\left(1+\tilde{q}_{h}^{a, b}(x, D)\right)^{-1} \exp \left[a \Lambda_{h}^{b}\right](x, D) f$

if $f \in H_{a, b}^{s}, s \in \boldsymbol{R}, a \geq 0,-1 \leq b \leq 1$ and $h \geq h_{p, a}$. This implies that

$$
\begin{aligned}
& Q_{h}^{a, b} \tilde{p}_{h}(x, D) f={ }^{R} \exp \left[-a \Lambda_{h}^{b}\right] \tilde{Q}_{h}^{a, b} \exp \left[a \Lambda_{h}^{b}\right] \tilde{p}_{h}{ }^{R} \exp \left[-a \Lambda_{h}^{b}\right] g \\
& ={ }^{R} \exp \left[-a \Lambda_{h}^{b}\right]\left(1+\tilde{q}_{h}^{a, b}\right)^{-1} \exp \left[a \Lambda_{h}^{b}\right] f=f, \\
& g=\left(1+\tilde{q}_{h}^{a, b}\right)^{-1} \exp \left[a \Lambda_{h}^{b}\right] f \in H^{m},
\end{aligned}
$$

if $f \in H_{a, b}^{s}$ and $a, b$ and $h$ satisfy (4.16). Similarly, we can prove $\tilde{p}_{h}(x, D) Q_{h}^{a, b} f=f$ if $f \in H_{a, b}^{(1-1 / k) l}$ and $a, b$ and $h$ satisfy (4.16). From Lemma 4.9 and (4.17) it follows that $H_{a, b}^{s} \subset H_{a^{\prime},-1}^{s}$ for $s \in \boldsymbol{R}, 0 \leq a \leq a^{\prime}$, $-1 \leq b \leq 1$ and $h \geq h_{p, a}$. Therefore, we have $Q_{h}^{a,-1} f=Q_{h}^{a, b} f$ if $f \in H_{a, b}^{(1-1 / \kappa) l}$ and $a, b$ and $h$ satisfy (4.16). Fix $h$ sufficiently large and define $Q_{h}$ by

$$
Q_{h} f=Q_{h^{\prime}}^{a^{\prime},-1} f-Q_{h^{\prime}}^{a^{\prime}-1}\left(\tilde{p}_{h}(x, D)-\tilde{p}_{h^{\prime}}(x, D)\right) Q_{h^{\prime}}^{a^{\prime}-1} f
$$

for $f \in H_{a^{\prime},-1}^{(1-1 / \kappa) l}$, where $a^{\prime}$ and $h^{\prime}$ satisfy (4.16). Then, by the same arguments as in the proof of Proposition 3.8 $Q_{h}$ does not depend on the choice of $a, a^{\prime}$ and $h^{\prime}$, and satisfies $\tilde{p}_{h}(x, D) Q_{h} f=Q_{h} \tilde{p}_{h}(x, D) f=f$ if $f \in L_{a^{\prime},-1}^{2}$ and $a^{\prime}$ satisfies (4.16). Moreover we have

$$
Q_{h} f-Q_{h^{\prime}}^{a^{\prime},-1} f \in H_{a^{\prime}, 1}^{m-(1-1 / \kappa) l}
$$

if $f \in H_{a^{\prime}-1}^{(1-1 / x) l}$ and $a^{\prime}$ and $h^{\prime}$ satisfy (4.16). Here we have modified $\hat{a}_{0}$ if necessary. So $Q_{h}$ maps continuously $L_{a^{\prime}, b}^{2}$ to $H_{a^{\prime}, b}^{m-(1-1 / \kappa) l}$ if $a^{\prime}$ and 
$b$ satisfy (4.16). To obtain this result we used $\Lambda_{h}^{b}$ instead of $\Lambda_{h}$. Let $\chi(x, \xi) \in \mathscr{E}^{(\kappa)}\left(T^{*} R^{n}\right)$ satisfy $\chi \subset \mathscr{C}_{1}$ for $z^{0}$. Here we have used the notation in the proof of Proposition 3.8. Choose $\phi(x) \in \mathscr{D}^{(x)}$ so that $\phi(x)=1$ in a neighborhood of $\left\{x \in \boldsymbol{R}^{n} ;(x, \xi) \in \mathscr{C}_{1}\right.$ for some $\left.\xi \in \boldsymbol{R}^{n}\right\}$, and define $Q$ by $Q f=Q_{h} \chi(x, D) \psi(x) f$. Let $\chi_{1}(x, \xi) \in \mathscr{E}^{(\kappa)}\left(T^{*} \boldsymbol{R}^{n}\right)$ satisfy $\chi_{1} \Subset \chi$ for $z^{0}$. Then we have

$$
\chi_{1}(x, D) p(x, D) Q f-\chi_{1}(x, D) f=\chi_{1}(\chi \psi-1) f+\chi_{1}\left(p-\tilde{p}_{h}\right) Q f \in L_{\kappa, \varepsilon_{0}^{\prime}}^{2}
$$

if $f \in H_{a^{\prime},-1}^{(1-1 / \kappa) l}$ and $a^{\prime}$ satisfies (4.16), where $\varepsilon_{0}^{\prime}=\hat{\varepsilon}_{0}^{\prime} A^{-1 / \kappa}$ and $\hat{\varepsilon}_{0}^{\prime}>0$. Define $\Lambda_{h}^{\prime}(x, \xi)$, replacing $\varphi_{1}(x, \xi)$ by $\varphi_{1}(x, \xi)-2\left(\left|x-x^{0}\right|^{2}+\left|\xi-\xi^{0}\right|^{2}\right) / 3$ in the construction of $\Lambda_{h}(x, \xi)$ in the proof of Lemma 4.3. Then we may assume that $\Lambda_{h}^{\prime}(x, \xi)$ satisfies

$$
\begin{aligned}
\Lambda_{h}(x, \xi) & \leq \Lambda_{h}^{\prime}(x, \xi)-\left(\left|x-x^{0}\right|^{2}+|\xi /| \xi\left|-\xi^{0}\right|^{2}\right)\langle\xi\rangle_{h}^{1 / x} / 2+C_{1}(h) \\
& \leq-\left(\varphi(x, \xi)+\left|x-x^{0}\right|^{2}+|\xi /| \xi\left|-\xi^{0}\right|^{2}\right)\langle\xi\rangle_{h}^{1 / \kappa}+C_{2}(h)
\end{aligned}
$$

for $(x, \xi) \in \mathscr{C}$ and $|\xi| \geq 1$. Let us prove that $Q$ is a left microlocal parametrix of $p$ on $H_{a\left(\Lambda_{h}^{\prime}+b w_{h}\right)}^{m}$. Let $f \in H_{a\left(\Lambda_{h}^{\prime}+b w_{h}\right)}^{m}$, and let $\tilde{\chi}(x, \xi) \in$ $\mathscr{E}^{(\kappa)}\left(T^{*} \boldsymbol{R}^{n}\right)$ be a positively homogeneous function of degree 0 in $\xi$ for $|\xi| \geq 1$ such that $0 \leq \tilde{\chi}(x, \xi) \leq 1$, supp $\chi \cap \operatorname{supp}(1-\chi) \cap\{|\xi|=1\}$ $\subset\left\{(x, \xi) \in T^{*} \boldsymbol{R}^{n} ; \tilde{\chi}(x, \xi)=1\right\}$ and supp $\tilde{\chi}(x, \xi)$ is included in a small conic neighborhood of supp $\chi \cap \operatorname{supp}(1-\chi) \cap\{|\xi|=1\}$. Assume that $a$ and $h$ satisfy (4.16). Then it follows from Proposition 2.8, Corollary 3 of Lemma 2.15 and its proof that

$$
(1-\tilde{\chi}(x, D)) \exp \left[a \Lambda_{h}^{b^{\prime}}\right](x, D)[p, \chi \psi] f \in L^{2}
$$

if $b, b^{\prime} \in[-1,1]$, modifying $\hat{a}_{0}$. On the other hand, Lemma 4.9 and (4.17) yield

$$
\begin{gathered}
\tilde{\chi}(x, D) \exp \left[a \Lambda_{h}^{b^{\prime}}\right](x, D)[p, \chi \psi] f=\tilde{\chi} \exp \left[a \Lambda_{h}^{b^{\prime}}\right]^{R} \exp \left[-a\left(\Lambda_{h}^{\prime}+b W_{h}\right)\right] \\
\times\left(1+\tilde{q}_{h}^{\prime a, b}\right)^{-1} \exp \left[a\left(\Lambda_{h}^{\prime}+b W_{h}\right)\right][p, \chi \psi] f \in L^{2}
\end{gathered}
$$

if $b^{\prime}-b<2 b_{0} \equiv \inf _{(x, \xi) \in \operatorname{supp} \bar{x}}\left(\left|x-x^{0}\right|^{2}+|\xi /| \xi\left|-\xi^{0}\right|^{2}\right) / 4$, where $1+\tilde{q}_{h}^{a, b}$ $(x, D)=\exp \left[a\left(\Lambda_{h}^{\prime}+b W_{h}\right)\right](x, D)^{R} \exp \left[-a\left(\Lambda_{h}^{\prime}+b W_{h}\right)\right](x, D)$. We may assume that $b_{0} \leq 2$. So we have $[p, \chi \psi] f \in L_{a, b+b_{0}}^{2}$ if $-1 \leq b \leq 1-b_{0}$. This gives $Q_{h}[p, \chi \psi] f \in H_{a, b_{0} / 2}^{m-(1-1 / \kappa) l}$ if $|b| \leq b_{0} / 2$. Modifying $\chi_{1}$, we can assume that

$$
\inf _{L>0} \sup _{(x, \xi) \in \operatorname{supp} x},|\xi| \geq L\left|\Lambda_{h}(x, \xi)\right|\langle\xi\rangle^{-1 / \kappa}<\varepsilon_{1} b_{0} / 8 .
$$

Then, by Lemma 4.9 and (4.17) we have 


$$
\chi_{1} Q_{h}[p, \chi \psi] f \in L_{\kappa, a \varepsilon_{1} b_{0} / 8}^{2} \text { if }|b| \leq b_{0} / 2 .
$$

This implies that

$$
\begin{aligned}
& \chi_{1}(x, D) Q p(x, D) f-\chi_{1}(x, D) f=-\chi_{1} Q_{h}[p, \chi \psi] f+\chi_{1}(\chi \psi-1) f \\
& \quad+\chi_{1} Q_{h}\left(p-\tilde{p}_{h}\right) \chi \psi f \in L_{\kappa, a \varepsilon_{0}}^{2}
\end{aligned}
$$

if $|b| \leq b_{0} / 2$, where $\hat{c}_{0}>0$. Now assume that $f \in \mathscr{D}^{* \prime}$ and $W F_{*}(f) \cap$ $\mathscr{C} \subset\{\varphi(x, \xi) \geq 0\}$. For a fixed $b$ with $-b_{0} / 2 \leq b<0$ there is $a_{f}>0$ such that

$$
\chi(x, D) \psi(x) f \in H_{a, b}^{(1-1 / \kappa) l} \cap H_{a\left(\Lambda_{h}^{\prime}+b w_{h}\right)}^{m}
$$

if $a \geq a_{f}$ when $*=(\kappa)$ and if $0<a \leq a_{f}$ when $*=\{\kappa\}$. In fact, let $\chi_{2}(x, \xi) \in \mathscr{E}^{(x)}\left(T^{*} \boldsymbol{R}^{n}\right)$ be a positively homogeneous function of degree 0 in $\xi$ for $|\xi| \geq 1$ such that $0 \leq \chi_{2}(x, \xi) \leq 1, \chi_{2}(x, \xi)=1$ if $|\xi| \geq 1$ and $\lim \sup _{\lambda \rightarrow+\infty}\left(\Lambda_{h}^{\prime}(x, \lambda \xi)+b W_{h}(x, \lambda \xi) / 4\right)(\lambda|\xi|)^{-1 / \kappa} \leq 0$, and $\chi_{2}(x, \xi)=0$ if $|\xi| \geq 1$ and $\lim \inf _{\lambda \rightarrow+\infty}\left(\Lambda_{h}^{\prime}(x, \lambda \xi)+b W_{h}(x, \lambda \xi) / 2\right)(\lambda|\xi|)^{-1 / k} \geq 0$. Since

$$
\begin{aligned}
& \lim \inf _{\lambda \rightarrow+\infty} \Lambda_{h}^{b}(x, \lambda \xi)(\lambda|\xi|)^{-1 / x} \\
& \leq \lim \inf _{\lambda \rightarrow+\infty}\left(\Lambda_{h}^{\prime}(x, \lambda \xi)+b W_{h}(x, \lambda \xi)\right)(\lambda|\xi|)^{-1 / x} \leq b \varepsilon_{1} / 4<0
\end{aligned}
$$

for $(x, \xi) \in \operatorname{supp} \chi_{2} \cap \mathscr{C}$ with $|\xi| \geq 1$, applying Lemma 4.9 and the Paley-Wiener theorem for $\mathscr{E}^{* \prime}$ (see, e. g., [18]), there is $a_{f}>0$ such that $\chi_{2}(x, D) \chi(x, D) \psi(x) f \in H_{a, b}^{(1-1 / \kappa) l} \cap H_{a\left(\Lambda_{h}^{\prime}+b w_{h}\right)}^{m}$ if $a \geq a_{f}$ when $*=(\kappa)$ and if $a>0$ when $*=\{\kappa\}$. On the other hand, we have (1$\left.\chi_{2}(x, D)\right) \chi(x, D) \psi(x) f \in \mathscr{E}^{*}$, since $\left\{\chi_{2}(x, \xi) \neq 1\right.$ and $\left.|\xi|=1\right\} \cap \mathscr{C} \Subset\{\varphi$ $(x, \xi)<0\}$. This proves (4.18), taking $a_{f}(>0)$ small enough when $*=\{\kappa\}$. Noting that $A$ can tend to zero when $*=(\kappa)$ and, therefore, $a$ can tend to $\infty$, we have $\chi_{1} p Q f-\chi_{1} f \in \mathscr{E}^{*}$ and $\chi_{1} Q p f-\chi_{1} f \in \mathscr{E} *$. In particular, if $f \in \mathscr{D}^{* \prime}$ and $W F_{*}(f) \cap \mathscr{C}=\emptyset$, then there is $a_{f}>0$ such that $\chi(x, D) \psi(x) f \in L_{a, 1}^{2}$ for any $a \geq 0$ when $*=(\kappa)$ and for $0 \leq a \leq a_{f}$ when $*=\{\kappa\}$. This implies that $Q f \in \mathscr{E}^{*}$ if $f \in \mathscr{D}^{* \prime}$ and $W F_{*}(f) \cap \mathscr{C}=\emptyset$. Therefore, we have just proved the following microlocal version of Holmgren's uniqueness theorem, which is necessary to prove that there is a conic neighborhood $\mathscr{C}_{3}$ of $z^{0}$ such that $W F_{*}(Q f) \cap \mathscr{C}_{3} \subset\{\varphi(z) \geq 0\}$ for $f \in \mathscr{D}^{*^{\prime}}$ with $W F_{*}(f) \subset\{\varphi(z) \geq 0\}$.

Proposition 4.10. Assume that $p(x, \xi)$ satisfies the condition $(\mathrm{A}-1)$ and (A-2) with $\kappa_{1}$ replaced by $\kappa(>1)$. Let $z^{0} \in T^{*} R^{n} \backslash 0$, and assume that $\varphi(z) \in C^{2}\left(T^{*} \boldsymbol{R}^{n} \backslash 0\right)$ is real-valued positively homogeneous of degree 0 
in $\xi$ and $\varphi\left(z^{0}\right)=0$ and that $p_{m}(z)$ is microhyperbolic with respect to $-H_{\varphi}\left(z^{0}\right)$ at $z^{0}$. Then $z^{0} \notin W F_{*}(u)$ if $u \in \mathscr{D}^{* \prime}, z^{0} \notin W F_{*}(p u)$ and $W F_{*}(u) \cap \mathscr{C} \cap\{\varphi(z)<0\}=\emptyset$ for a conic neighborhood $\mathscr{C}$ of $z^{0}$. Here * denotes $(\kappa)$ or $\{\kappa\}$.

Let $f \in \mathscr{D}^{*^{\prime}}$ satisfy $W F_{*}(f) \cap \mathscr{C} \subset\{\varphi(z) \geq 0\}$. Then $Q f \in H_{a, b}^{(1-1 / \kappa) l}$ for a fixed $b$ with $-b_{0} / 2 \leq b<0$, and $a \geq a_{f}$ when $*=(\kappa)$ and $0<a \leq a_{f}$ when $*=\{\kappa\}$, if $a$ satisfies (4.16). Therefore, we have $W F_{*}(Q f) \cap$ $\left\{\tilde{\varphi}_{2}(x, \xi)<3 b\right\}=\emptyset$, since $\inf _{L>0} \sup \left\{\Lambda_{h}^{b}(x, \xi)|\xi|^{-1 / \kappa} ; \tilde{\varphi}_{2}(x, \xi)<3 b\right.$ and $|\xi| \geq L\} \geq-b$, where $\tilde{\varphi}_{2}(x, \xi)=\varphi_{2}(x, \xi /|\xi|)$ and $\varphi_{2}(z)$ is defined by (4.10). We may assume that $-H_{\varphi}(z)$ and $-H_{\tilde{\varphi}_{2}}(z)$ belong to $\Gamma\left(p_{m z}, \vartheta\right)$ for $z \in \mathscr{C}_{4} \equiv\left\{(x, \xi) ;\left|x-x^{0}\right|^{2}+|\xi /| \xi\left|-\xi^{0}\right|^{2} \leq r_{0}^{2}\right\}$ and $W F_{*}$ $(P Q f-f) \cap \mathscr{C}_{4}=\emptyset$, where $r_{0}>0$. Let $\zeta(x, \xi) \in C^{2}\left(T^{*} \boldsymbol{R}^{n} \backslash 0\right)$ be a realvalued positively homogeneous function in $\xi$ such that $0 \leq \zeta(x, \xi) \leq 1$, and $\zeta(x, \xi)=1$ if $\left|x-x^{0}\right|^{2}+|\xi /| \xi\left|-\xi^{0}\right|^{2} \leq r_{1}^{2}$ and $\zeta(x, \xi)=0$ if $\left|x-x^{0}\right|^{2}$ $+|\xi /| \xi\left|-\xi^{0}\right|^{2} \geq 4 r_{1}^{2}$, where $0<r_{1} \quad\left(\leq r_{0} / 2\right)$. Then we may assume that $\left|H_{\zeta}(x, \xi)\right|=O\left(r_{1}^{-1}\right)$ for $|\xi|=1$. Since $\left|\varphi(x, \xi)-\tilde{\varphi}_{2}(x, \xi)\right|=O(\mid x$ $\left.-\left.x^{0}\right|^{2}+|\xi /| \xi\left|-\xi^{0}\right|^{2}\right)$, we have $-H_{\varphi_{\theta}}(z) \in \Gamma\left(p_{m z}, \vartheta\right)$ for $z \in \mathscr{C}_{4}$ and $\theta \in$ $[0,1]$, if $|b|$ and $r_{1}$ are sufficiently small, where $\varphi_{\theta}(z)=\theta\{\zeta(z) \varphi(z)$ $\left.+(1-\zeta(z))\left(\tilde{\varphi}_{2}(z)-3 b\right)\right\}+(1-\theta)\left(\tilde{\varphi}_{2}(z)-3 b\right)$. Now assume that there is $\theta \in[0,1)$ such that $W F_{*}(Q f) \cap\left\{z \in \mathscr{C}_{4} ; \varphi_{\theta}(z)=0\right\} \neq \emptyset$. We set $\theta_{0}=\inf \left\{\theta \in[0,1) ; W F_{*}(Q f) \cap\left\{z \in \mathscr{C}_{4} ; \varphi_{\theta}(z)=0\right\} \neq \emptyset\right\}$. Then, $W F_{*}$ $(p Q f) \cap\left\{z \in \mathscr{C}_{4} ; \varphi_{\theta_{0}}(z)=0\right\}=\emptyset$ and $W F_{*}(Q f) \cap\left\{z \in \mathscr{C}_{4} ; \varphi_{\theta_{0}}(z)<0\right\}$ $=\emptyset$. Therefore, Proposition 4.10 implies that $W F_{*}(Q f) \cap\left\{z \in \mathscr{C}_{4}\right.$; $\left.\varphi_{\theta_{0}}(z)=0\right\}=\emptyset$, which contradicts the definition of $\theta_{0}$. This proves that $W F_{*}(Q f) \cap \mathscr{C}_{3} \subset\{\varphi(z) \geq 0\}$, where $\mathscr{C}_{3}=\left\{(x, \xi) ;\left|x-x^{0}\right|^{2}+|\xi /| \xi \mid\right.$ $\left.-\left.\xi^{0}\right|^{2} \leq r_{1}^{2}\right\}$. Thus we have the following

Theorem 4.11. Let the hypothesis of Proposition 4.10 be satisfied, and let $\mathscr{C}$ be a conic neighborhood of $z^{0}$. Then there are a continuous operator $Q: \mathscr{D}^{*^{\prime}} \rightarrow \mathscr{D}^{*^{\prime}}$ and a conic neighborhood $\mathscr{C}_{1}$ of $z^{0}$ such that

$$
\begin{aligned}
& z^{0} \oplus W F_{*}(p Q f-f) \cup W F_{*}(Q p f-f), \\
& W F_{*}(Q f) \cap \mathscr{C}_{1} \subset\{\varphi(z) \geq 0\},
\end{aligned}
$$

if $f \in \mathscr{D}^{*^{\prime}}$ and $W P_{*}(f) \cap \mathscr{C} \subset\{\varphi(z) \geq 0\}$. Moreover, $z^{0} \notin W F_{*}(Q f)$ if $z^{0} \notin W F_{*}(f)$ 。 
Remark. (i) The theorem implies that (MCP) is microlocally wellposed in $\mathscr{D}^{*^{\prime}}$ at $z^{0}$ modulo $\mathscr{E}^{*}$. (ii) By Theorems 1.5 and 4.11, (MCP) can be solved globally modulo $\mathscr{E}^{*}$ under reasonable assumptions.

\section{§5. Proof of Theorem 1.4 and Some Remarks}

Let us begin with some remarks on existence of time functions.

Proposition 5.1. Let $z^{0}=\left(x^{0}, \xi^{0}\right) \in T^{*} \boldsymbol{R}^{n} \backslash 0$ and $\vartheta \in T_{z}{ }^{0}\left(T^{*} \boldsymbol{R}^{n}\right)$, and assume that $p_{m}(x, \xi)$ is microhyperbolic with respect to $\vartheta$ at $z^{0}$. Then the following conditions are equivalent: (i) There are a conic neighborhood $\mathscr{C}$ of $z^{0}$ and a time function for $p_{m}$ in $\mathscr{C}$. (ii) There is $\tilde{\vartheta} \in T_{z^{0}}\left(T^{*} \boldsymbol{R}^{n}\right)$ such that $p_{m}$ is microhyperbolic with respect to $\tilde{\vartheta}$ at $z^{0}$ and $\sigma\left(r_{0}, \tilde{\vartheta}\right)=0$, where $r_{0}=\sum_{j=1}^{n} \xi_{j}^{0}\left(\partial / \partial \xi_{\jmath}\right)$. (iii) There is $\tilde{\vartheta} \in T_{z^{0}}\left(T^{*} \boldsymbol{R}^{n}\right)$ such that $p_{m}$ is microhyperbolic with respect to $\tilde{\vartheta}$ at $z^{0}$ and $\pm r_{0} \notin \Gamma\left(p_{m z}{ }^{0}, \tilde{\vartheta}\right)^{\sigma}$.

Proof. Let $t(x, \xi)$ be a time function for $p_{m}$ in $\mathscr{C}$. Then $p_{m}$ is microhyperbolic with respect to $-H_{t}\left(z^{0}\right)$ at $z^{0}$ and $\sum_{j=1}^{n}\left(\partial t / \partial \xi_{j}\right)(x, \xi)$ $\xi_{j}=0$, i. e., $\sigma\left(r_{0},-H_{t}\left(z^{0}\right)\right)=0$. This proves that the condition (i) implies the condition (ii). It is obvious that the condition (ii) implies the condition (iii). Assume that the condition (iii) holds. Then there are $\vartheta^{j} \in \Gamma\left(p_{m z^{0}}, \tilde{\vartheta}\right) \quad(j=1,2)$ such that $(-1)^{j} \sigma\left(\vartheta^{j}, r^{0}\right)>0$. Therefore, there is $\vartheta^{0} \in \Gamma\left(p_{m z^{0}}, \tilde{\vartheta}\right)$ such that $\sigma\left(\vartheta^{0}, r^{0}\right)=0$. Then $t(x, \xi)$ $\equiv \sigma\left(\vartheta^{0},\left(x-x^{0},\left|\xi^{0}\right| \xi /|\xi|-\xi^{0}\right)\right)$ is a time function for $p_{m}$ in a conic neighborhood of $z^{0}$.

Q. E. D.

We assume that the hypotheses of Theorem 1.4 be satisfied. We shall prove Theorem 1.4 by the same arguments as in [30], using Proposition 4. 10 (and Theorem 4. 11). If $p_{m}\left(z^{0}\right) \neq 0$, Proposition 3.8 implies that $z^{0} \notin W F_{*}(u)$ when $u \in \mathscr{D}^{* 1^{\prime}}$ and $z^{0} \notin W F_{*}(p u)$. So, in Theorem $1.4 p_{m}(z)$ must vanish at $z^{0}$. If $\Gamma\left(p_{m z^{0}}, \vartheta\left(z^{0}\right)\right)^{\sigma}$ contains $r_{0} \equiv \sum_{j=1}^{n} \xi_{j}^{0}\left(\partial / \partial \xi_{j}\right)$ or $-r_{0}$, then Theorem 1.4 is trivial.

Proposition 5.2. Let $z^{0}=\left(x^{0}, \xi^{0}\right) \in \Omega$ and $\left|\xi^{0}\right|=1$, and let $M$ be a compact subset of $\Gamma\left(p_{m z^{0}}, \vartheta\left(z^{0}\right)\right)$. Assume that $p_{m}\left(z^{0}\right)=0$ and $\pm r_{0} \notin$ 
$\Gamma\left(p_{m z^{0}}, \vartheta\left(z^{0}\right)\right)^{\sigma}$ and that $\vartheta^{0} \in \Gamma\left(p_{m z^{0}}, \vartheta\left(z^{0}\right)\right)$ and $\sigma\left(\vartheta^{0}, r_{0}\right)=0$. Then there is $t_{0}>0$ such that

$$
\begin{gathered}
W F_{*}(u) \cap\left\{(x, \xi) \in z^{0}-M^{\sigma} ; \sigma\left(\left(x-x^{0}, \xi /|\xi|-\xi^{0}\right), \vartheta^{0}\right)=t\right\} \neq \emptyset \\
\text { for } 0 \leq t \leq t_{0}
\end{gathered}
$$

if $u \in \mathscr{D}^{* 1^{\prime}}$ and $z^{0} \in W F_{*}(u) \backslash W F_{*}(p u)$.

Proof. We may assume that $u \in \mathscr{E}^{* 1^{\prime}}$ and that $\vartheta^{0} \in \stackrel{\circ}{M}$, i. e., $\sigma\left(\vartheta^{0}\right.$, $\delta z)>0$ for $\delta z \in M^{\sigma} \backslash\{0\}$. Let $M_{1}$ be a compact subset of $\Gamma\left(p_{m z}, \vartheta\left(z^{0}\right)\right)$ such that $M \Subset M_{1}$. Then there are a neighborhood $\mathscr{U}$ of $z^{0}$ and $t_{0}>0$ such that $W F_{*}(p u) \cap \mathscr{U}=\emptyset, p_{m}$ is microhyperbolic with respect to $\vartheta^{0}$ at $z \in \mathscr{U}, M_{1} \subset \Gamma\left(p_{m z}, \vartheta\right)$ for $z \in \mathscr{U}, t(x, \xi)$ is a time function for $p_{m}$ in a conic neighborhood of $\mathscr{U}$ and $\left\{z \in z^{0}-M^{\sigma} ;-t_{0} \leq t(x, \xi)\right.$ $\leq 0\} \Subset \mathscr{U}$, where $t(x, \xi)=\sigma\left(\vartheta^{0},\left(x-x^{0}, \xi /|\xi|-\xi^{0}\right)\right)$. Now assume taht $W F_{*}(u) \cap\left\{z \in z^{0}-M^{\sigma} ; t(x, \xi)=-t_{1}\right\}=\emptyset$ for some $t_{1}$ with $0<t_{1} \leq t_{0}$. We can assume without loss of generality that $\xi^{0}=(0, \cdots, 0,1)$. We denote by $S^{*} \boldsymbol{R}^{n}\left(\simeq \boldsymbol{R}^{n} \times S^{n-1}\right)$ the cosphere bundle over $\boldsymbol{R}^{n}$ and we use inhomogeneous local coordinates $(x, q)$. Let $\tau: T^{*} \boldsymbol{R}^{n} \backslash 0 \rightarrow S^{*} \boldsymbol{R}^{n}$ be the canonical map defined as $(x, \xi) \mapsto\left(x,-\xi_{1} / \xi_{n}, \cdots,-\xi_{n-1} / \xi_{n}\right)$ for $(x, \xi)$ $\in T^{*} \boldsymbol{R}^{n} \backslash 0$ with $\xi_{n} \neq 0$. The map $\tau$ induces a map $d \tau_{z}: T_{z}\left(T^{*} \boldsymbol{R}^{n} \backslash 0\right)$ $\ni(\delta x, \delta \xi) \mapsto(\delta x, \delta q) \in T_{\tau(z)}\left(S^{*} \boldsymbol{R}^{n}\right)$, where $\delta q_{j}=-\xi_{n}^{-1}\left(\delta \xi_{j}+q_{j} \delta \xi_{n}\right) \quad(1 \leq j$ $\leq n-1), z=(x, \xi)$ and $q_{j}=-\xi_{j} / \xi_{n}(1 \leq j \leq n-1)$. Since $\pm r_{0} \notin M^{\sigma}$ and $M^{\sigma}$ is a closed proper convex cone, modifying $\mathscr{U}$ if necessary, there is a closed convex cone $K$ with its vertex at the origin in $R^{2 n-1}\left(\simeq T_{\tau(z)}\left(S^{*} \boldsymbol{R}^{n}\right)\right)$ such that

$$
\begin{aligned}
& d \tau_{z}\left(M^{\sigma}\right) \supset K \supset d \tau_{z}\left(M_{1}^{o}\right) \quad \text { for } z \in \mathscr{U}, \\
& \tau\left(z^{0}-M^{\sigma}\right) \cap \tau(\mathscr{U}) \supset\left(\tau\left(z^{0}\right)-K\right) \cap \tau(\mathscr{U}) .
\end{aligned}
$$

Then there are $\varepsilon>0$ and $\hat{z} \in \stackrel{\circ}{K}$ such that

$$
\tau\left(W F_{*}(u)\right) \cap K^{\prime} \cap\left\{\tau(z) ;-t_{1}+\varepsilon \geq t(z) \geq-t_{1}\right\}=\emptyset,
$$

where $K^{\prime}=\tau\left(z^{0}\right)+\hat{z}-K$. Let $\phi(x, \xi) \in \mathscr{E}^{\left(\kappa_{1}\right)}\left(T^{*} \boldsymbol{R}^{n}\right)$ be a positively homogeneous functions of degree 0 in $\xi$ for $|\xi| \geq 1 / 2$ such that $\phi(x, \xi)=1$ if $(x, \xi) \in \mathscr{C},|\xi| \geq 1 / 2$ and $t(x, \xi) \geq-t_{1}+\varepsilon$, and $\psi(x, \xi)$ $=0$ if $(x, \xi) \in \mathscr{C},|\xi| \geq 1 / 2$ and $t(x, \xi) \leq-t_{1}$, where $\mathscr{C}$ is a conic neighborhood of $\mathscr{U}$. We set $v=\psi(x, D) u$ and $g=p(x, D) v \quad(=\phi p u+$ $[p, \psi] u)$. Then, 
$W F_{*}(g) \cap \mathscr{U}=W F_{*}([p, \psi] u) \cap \mathscr{U} \subset\left\{z \in \mathscr{U} ;-t_{1}+\varepsilon \geq t(z) \geq-t_{1}\right\}$, $\tau\left(W F_{*}(g)\right) \cap K^{\prime}=\emptyset, W F_{* 1}(v) \cap \mathscr{U} \subset\left\{z \in \mathscr{U} ; t(z) \geq-t_{1}\right\}$.

We may assume that the boundary $\partial\left(K^{\prime} \cap T\right)$ of $K^{\prime} \cap T$ in $S^{*} R^{n} \cap T$ is smooth, where $T=\left\{\tau(z) ; t(z)=-t_{1}\right\}$. Let $S$ be a $C^{2}$ hypersurface in $S^{*} R^{n}$ such that $S \cap T=\partial\left(K^{\prime} \cap T\right)$ and one of the normals $(\delta x, \delta q)$ at each point on $S \cap \tau(\mathscr{U})$ belongs to $K^{*}$, where $K^{*}=\{(\delta x, \delta q)$; $\delta x \cdot \delta \tilde{x}+\delta q \cdot \delta \tilde{q} \geq 0$ for any $(\delta \tilde{x}, \delta \tilde{q}) \in K\}$. The family of hypersurfaces $S$ with the above properties sweeps out the region $\AA^{\prime} \cap\{\tau(z) ; t(z) \geq$ $\left.-t_{1}\right\}$ (see [12]). Assume that $\varphi \in C^{2}\left(T^{*} \boldsymbol{R}^{n} \backslash 0\right)$ is real-valued, positively homogeneous of degree 0 in $\xi, \tau^{-1}(S) \cap \mathscr{U}=\{z \in \mathscr{U} ; \varphi(z)=0\}, d \tau_{z}\left(H_{\varphi}\right.$ $(z)) \in K^{*}$ on $\tau^{-1}(S) \cap \mathscr{U}$ and $W F_{* 1}(v) \cap\{z \in \mathscr{U} ; \varphi(z)<0\}=\emptyset$. We need the following

Lemma 5. 3. (Lemma 3. 1 in [30]). For $z^{1}=\left(x^{1}, \xi^{1}\right) \in \tau^{-1}(S) \cap \mathscr{U}$, we have $-H_{\varphi}\left(z^{1}\right) \in \Gamma\left(p_{m z^{1}}, \vartheta^{0}\right)$.

From Lemma 5.3 and Theorem 4.11 it follows that $z^{1} \notin W F_{* 1}(Q g$ -v) for $z^{1} \in \tau^{-1}\left(S \cap K^{\prime}\right)$, where $Q$ is as defined in Theorem 4.11, replacing $z^{0}$ and $\kappa$ by $z^{1}$ and $\kappa_{1}$, respectively. From the proof of Theorem 4.11 it follows that $Q: \mathscr{D}^{*^{\prime}} \rightarrow \mathscr{D}^{*^{\prime}}$ and $Q$ satisfies the assertions in Theorem 4.11 with $z^{0}$ replaced by $z^{1}$. Therefore, we have $z^{1} \notin W F_{*}(Q g)$ and $W F_{*}(v) \cap \tau^{-1}\left(S \cap K^{\prime}\right)=\emptyset$. The method of sweeping out in [12] shows that $z^{0} \notin W F_{*}(v)$. This proves Proposition 5. 2.

Q.E.D.

From the same arguments as in the proof of Theorem 3.3 in [31], it follows that for every $z^{0} \in \Omega$ there are neighborhood $\mathscr{U}\left(z^{0}\right)$ $(\subset \Omega)$ of $z^{0}$ and $t\left(z^{0}\right)>0$ such that for any $z^{1} \in \mathscr{U}\left(z^{0}\right)$ there is a Lipschitz continuous function $z(t)$ defined on $\left(-t\left(z^{0}\right), 0\right]$ with values in $\Omega$ satisfying $z(t) \in W F_{*}(u)$ for $t \in\left(-t\left(z^{0}\right), 0\right],(d / d t) z(t) \in \Gamma\left(p_{m z(t)}\right.$, $\vartheta(z(t)))^{\sigma} \cap\{\delta z ;|\delta z|=1\}$ for a.e. $t \in\left(-t\left(z^{0}\right), 0\right]$ and $z(0)=z^{1}$ if $u \in \mathscr{D}^{* 1^{\prime}}, z^{1} \in W F_{*}(u)$ and $W F_{*}(p u) \cap \Omega=\emptyset$. Therefore, by the same arguments as in the proof of extension theorem in theory of ordinary differential equations, we can prove Theorem 1.4. 


\section{References}

[1] Aoki, T., The exponential calculus of microdifferential operators of infinite order. I-IV, Proc. Japan Acad., 58 (1982), 58-61 and 154-157; 59 (1983), 79-82 and 186187.

[2 ] Atiyah, M. F., Bott, R., and Gårding, L., Lacunas for hyperbolic differential operators with constant coefficients, I, Acta Math., 124 (1970), 109-189.

[3] Bony, J. M., and Schapira, P., Solutions "hyperfonctions du probleme de Cauchy, Hyperfunctions and Pseudo-differential Equations, Lecture Notes in Math. 287, Springer, 1973.

[4] Boutet de Monvel, L., Opérateurs pseudo-différentiels analytiques et opérateurs d'ordre infini, Ann. Inst. Fourier, Grenoble, 22 (1972), 229-268.

[5] Bronshtein, M. D., The Cauchy problem for hyperbolic operators with variable multiple characteristics, Trudy Moskov. Mat Obšč., 41 (1980), 83-99.

[6] Calderon, A., and Vaillancourt, R., A class of bounded pseudodifferential operators, Proc. Nat. Acad. Sci. USA, 69 (1972), 1185-1187.

[7] Cordes, H., On compactness of commutators of multiplications and convolutions, and boundedness of pseudo-differential operators, J. Funct. Anal., 18 (1975), 115-131.

[8] Hashimoto, S., Matsuzawa, T., and Morimoto, Y., Opérateurs pseudodifferentiels et classes de Gevrey, Comm. in Partial Differential Equations, 8 (1983), 1277-1289.

[9] Hörmander, L., Pseudo-differential operators and hypoelliptic equations, Proc. Symposium on Singular Integrals, Amer. Math. Soc., 10 (1967), 138-183.

[10] Uniqueness theorems and wave front sets for solutions of linear differential equations with analytic coefficients, Comm. Pure Appl. Math., 26 (1971), 671-704.

[11] The Analysis of Linear Partial Differential Operators I, Springer, BerlinHeidelberg-New York-Tokyo, 1983.

[12] John, F., On linear partial differential equations with analytic coefficients, unique continuation of data. Comm. Pure Appl. Math., 2 (1949), 209-254.

[13] Kajitani, K., Cauchy problem for nonstrictly hyperbolic systems in Gevrey classes, $J$. Math. Kyoto Univ., 23 (1983), 599-616.

[14] The Cauchy Problem for uniformly diagonalizable hyperbolic systems, Proc. Hyperbolic Equations and Related Topics, Taniguchi Symposium 1984, Kinokuniya, Tokyo, 101123.

[15] Wellposedness in Gevrey class of the Cauchy problem for hyperbolic operators, Bull. Sci. Math., 111 (1987), 415-438.

[16] Kashiwara, M., and Kawai, T., Micro-hyperbolic pseudo-differential operators I, $J$. Math. Soc. Japan, 27 (1975), 359-404.

[17] Kato, T., Boundedness of some pseudo-differential operators, Osaka J. Math., 13 (1976), $1-9$.

[18] Komatsu, H., Ultradistributions, I, Structure theorems and a characterization, J. Fac. Sci. Univ. Tokyo Sect. IA Math., 20 (1973), 25-105.

[19] Kumano-go, H., Pseudo-Differential Operators, MIT Press, Cambridge, 1982.

[20] Lascar, R., Propagation des singularités des solutions d'equations pseudo-différentielles à caractéristiques de multiplicités variables, Lecture Notes in Math. 856, Springer-Verlag, 1981.

[21] Leray, J., Hyperbolic Differential Equations, Princeton Univ. Press, Princeton, 1952.

[22] Mizohata, S., On the Cauchy Problem, Academic Press, New York, 1986.

[23] Rodino, L. and Zanghirati, L., Pseudo differential operators with multiple characteristics and Gevrey singularities, Comm. in Partial Differential Equations, 11 (1986), 673-711. 
[24] Taniguchi, K., Pseudo-differential operators acting on ultradistributions, Math.Japonica, 30 (1985), 719-741.

[25] - On multi-products of pseudo-differential operators in Gevrey classes and its application to Gevrey hypoellipticity, Proc. Japan Acad., 61 (1985), 291-293.

[26] Treves, F., Introduction to Pseudodifferential and Fourier Integral Operators I, Plenum Press, New York and London, 1980.

[27] Uchikoshi, K., Construction of solutions of microhyperbolic pseudodifferential equations, J. Math. Soc. Japan, 40 (1988), 289-318.

[28] Wakabayashi, S., The Cauchy problem for operators with constant coefficient hyperbolic principal part and propagation of singularities, Japan. J. Math., 6 (1980), 179-228.

[29] - Analytic singularities of solutions of the hyperbolic Cauchy problem, Proc. Japan Acad., 59 (1983), 449-452.

[30] - Singularities of solutions of the Cauchy problem for symmetric hyperbolic systems, Comm. in Partial Differential Equations, 9 (1984), 1147-1177.

[31] Singularities of solutions of the Cauchy problem for hyperbolic systems in Gevrey classes, Japan J. Math., 11 (1985), 157-201.

[32] — Remarks on hyperbolic polynomials, Tsukuba J. Math., 10 (1986), 17-28.

[33] Generalized Hamilton flows and singularities of solutions of the hyperbolic Cauchy problem, Proc. Hyperbolic Equations and Related Topics, Taniguchi Symposium 1984, Kinokuniya, Tokyo. pp. 415-423. 
